\title{
First tephrostratigraphic results of the DEEP site record from Lake Ohrid (Macedonia and Albania)
}

\author{
Niklas Leicher $^{1}$, Giovanni Zanchetta ${ }^{2}$, Roberto Sulpizio ${ }^{3,4}$, Biagio Giaccio $^{5}$, Bernd Wagner ${ }^{1}$, Sebastien Nomade $^{6}$, \\ Alexander Francke ${ }^{1}$, and Paola Del Carlo ${ }^{7}$ \\ ${ }^{1}$ Institute of Geology and Mineralogy, University of Cologne, Zuelpicher Str. 49a, Cologne, Germany \\ ${ }^{2}$ Dipartimento di Scienze della Terra, University of Pisa, Via S. Maria 53, Pisa, Italy \\ ${ }^{3}$ Dipartimento di Scienze della Terra e Geoambientali, University of Bari, Via Orabona 4, Bari, Italy \\ ${ }^{4}$ Istituto per la Dinamica dei Processi Ambientali (IDPA) CNR, Via M. Bianco 9, Milan, Italy \\ ${ }^{5}$ Istituto di Geologia Ambientale e Geoingegneria, CNR, Via Salaria km 29300, Monterotondo, Rome, Italy \\ ${ }^{6}$ Laboratoire des sciences du climat et de l'environnement, UMR 8212, CEA/CNRS/UVSQ et Université \\ Paris-Saclay, Gif-Sur-Yvette, France \\ ${ }^{7}$ Istituto Nazionale di Geofisica e Vulcanologia, Sezione di Pisa, Via della Faggiola 32, Pisa, Italy
}

Correspondence to: Niklas Leicher (n.leicher@uni-koeln.de)

Received: 14 August 2015 - Published in Biogeosciences Discuss.: 17 September 2015

Revised: 14 February 2016 - Accepted: 10 March 2016 - Published: 13 April 2016

\begin{abstract}
A tephrostratigraphic record covering the Marine Isotope Stages (MIS) 1-15 was established for the DEEP site record of Lake Ohrid (Macedonia and Albania). Major element analyses (energy dispersive spectroscopy (EDS) and wavelength-dispersive spectroscopy (WDS)) were carried out on juvenile fragments extracted from 12 tephra layers (OH-DP-0115 to OH-DP-2060). The geochemical analyses of the glass shards of all of these layers suggest an origin in the Italian volcanic provinces. They include the Y-3 (OH-DP-0115, 26.68-29.42 ka cal BP), the Campanian Ignimbrite-Y-5 (OH-DP-0169, $39.6 \pm 0.1 \mathrm{ka})$, and the X6 (OH-DP-0404, 109 $\pm 2 \mathrm{ka}$ ) from the Campanian volcanoes, the P-11 of Pantelleria (OH-DP-0499, $133.5 \pm 2 \mathrm{ka}$ ), the Vico B (OH-DP-0617, $162 \pm 6 \mathrm{ka}$ ) from the Vico volcano, the Pozzolane Rosse (OH-DP-1817, $457 \pm 2 \mathrm{ka}$ ) and the Tufo di Bagni Albule (OH-DP-2060, $527 \pm 2 \mathrm{ka}$ ) from the Colli Albani volcanic district, and the Fall A (OH-DP$2010,496 \pm 3 \mathrm{ka}$ ) from the Sabatini volcanic field. Furthermore, a comparison of the Ohrid record with tephrostratigraphic records of mid-distal archives related to the Mediterranean area allowed the recognition of the equivalents of other less known tephra layers, such as the TM24a-POP2 (OH-DP-0404, $102 \pm 2 \mathrm{ka}$ ) recognized in the Lago Grande di Monticchio and the Sulmona Basin, the CF-V5-PRAD3225 (OH-DP-0624, ca. $163 \pm 22 \mathrm{ka}$ ) identified in the Campo Fe-
\end{abstract}

lice Basin and the Adriatic Sea, the SC5 (OH-DP-1955, $493.1 \pm 10.9 \mathrm{ka})$ recognized in the Mercure Basin, and the A11/12 (OH-DP-2017, 511 $\pm 6 \mathrm{ka}$ ) sampled at the Acerno Basin, whose specific volcanic sources are still poorly constrained. Additionally, one cryptotephra (OH-DP-0027) was identified by correlation of the potassium X-ray flourescence (XRF) intensities from the DEEP site with those from a short core of a previous study from Lake Ohrid. In these cores, a maximum in potassium is caused by glass shards, which were correlated with the Mercato tephra (8.43-8.63 kacal BP) from Somma-Vesuvius. The tephrostratigraphic work presented here allows, for the first time, the extension of a consistent part of the Middle Pleistocene tephrostratigraphy of Italian volcanoes as far as the Balkans. The establishment of the tephrostratigraphic framework for the Lake Ohrid record provides important, independent tie points for the age-depth model of the DEEP site sequence, which is a prerequisite for palaeoclimatic and palaeoenvironmental reconstructions. Furthermore, this age-depth model will help to improve and re-evaluate the chronology of other, both undated and dated tephra layers from other records. Thus, the Lake Ohrid record may potentially become the template for the central Mediterranean tephrostratigraphy, especially for the hitherto poorly known and explored lower Middle Pleistocene period. 


\section{Introduction}

Volcanic explosive eruptions produce pyroclastic material, called tephra (Gr. $\tau \varepsilon \varphi \rho \alpha$, "ash"), which is ejected into the atmosphere and distributed by the prevailing wind systems. Tephra settles down from the atmosphere in a relatively short time (days-weeks) as isochronous event marker horizons into all kind of geological archives downwind of the volcano. By determining the unique geochemical and physical fingerprint of such a tephra horizon, tephra layers (from different archives) can be identified, characterized, and correlated with each other in order to obtain a tephrostratigraphic framework. If tephra horizons can be dated directly (e.g. ${ }^{40} \mathrm{Ar} /{ }^{39} \mathrm{Ar}$ ) or indirectly (e.g. ${ }^{14} \mathrm{C}$ dating on overlying or underlying sediments, varve counting, age modelling) and correlated with tephra horizons in other archives, the ages can also be transferred to these other archives.

Italian volcanism was characterized by an intense explosive activity during the entire Quaternary (Peccerillo, 2005). Consequently, the surrounding Mediterranean region became an ideal setting for tephrochronological studies (tephrostratigraphy and tephrochronometry; cf. SarnaWojcicki, 2013), which represents a key tool for a wide spectrum of Quaternary science subjects (e.g. Lowe, 2011). After Keller et al. (1978) set up the first tephrostratigraphic scheme for the central Mediterranean region, numerous studies on marine and terrestrial archives have spatially and temporally extended and improved this initial stratigraphy for the Holocene and Late Pleistocene (Paterne et al., 1986, 1988, 2008; Vezzoli, 1991; Calanchi et al., 1998; Narcisi and Vezzoli, 1999; Siani et al., 2004; Calanchi and Dinelli, 2008; Zanchetta et al., 2011; Tamburrino et al., 2012; Insinga et al., 2014; Satow et al., 2015; Tomlinson et al., 2015). Despite this noticeable progress over the last decades, tephrochronological work in the period before $200 \mathrm{ka}$ is still challenging due to incomplete knowledge of the eruption history and limited geochemical analysis. Some records from the Italian Peninsula cover specific intervals of the Early to Middle Pleistocene and can be used as proximal (Karner et al., 2001; Rouchon et al., 2008; Marra et al., 2009, 2014; Palladino et al., 2010; Giaccio et al., 2013a) or relatively distal (Karner et al., 1999; Munno and Petrosino, 2007; Roulleau et al., 2009; Russo Ermolli et al., 2010; Giaccio et al., 2013b, 2014, 2015; Petrosino et al., 2014a, b, 2015; Sagnotti et al., 2014) archives of deposits from volcanic complexes. Sediment records continuously spanning more than $200 \mathrm{kyr}$ are extremely rare in the Mediterranean region. To date, there are only two continuous records covering the entire Middle and parts of the early Pleistocene of the Mediterranean region, which are the Calabrian Ridge core KC01B (Lourens, 2004; Insinga et al., 2014) and the peat record from Tenaghi Philippon, Greece (Tzedakis, 1993; St. Seymour et al., 2004; Pross et al., 2007). However, both records are limited in the tephrostratigraphy to the Holocene and upper Middle Pleistocene.
Lake Ohrid is located on the Balkan Peninsula and is one of the oldest lakes of Europe (Wagner et al., 2014). Over 1.2 Myr of continuous sediments were recovered from Lake Ohrid during the ICDP (International Continental Scientific Drilling Program) deep drilling campaign SCOPSCO (Scientific Collaboration on Past Speciation Conditions in Lake Ohrid). Previous tephrochronological studies on sediment cores from Lake Ohrid covered the last $135 \mathrm{kyr}$ and revealed the lake's unique potential as a distal tephra archive of Italian volcanoes (e.g. Sulpizio et al., 2010).

Here, we present first tephrostratigraphic and tephrochronological results of the uppermost $247.8 \mathrm{~m}$ composite depth (mcd) of the main drill site (DEEP site) in the central part of the lake, which covers the last $637 \mathrm{kyr}$ continuously (Francke et al., 2016). The correlation of the discovered tephra layers to known and dated equivalent tephra horizons from proximal and distal archives enables dating of the Lake Ohrid succession. The transfer of these ages to the Lake Ohrid record provides important, independent tie points for an age-depth model complemented by orbital tuning (Francke et al., 2016), which is a precondition for environmental and climate reconstructions. The correlation of tephra layers between different geographical archives, both terrestrial and marine, is crucial for a synchronization of palaeoclimatic and palaeoenvironmental changes on a regional and global scale.

\section{Regional setting}

Lake Ohrid $\left(40^{\circ} 54^{\prime}-41^{\circ} 10^{\prime} \mathrm{N}, 20^{\circ} 38^{\prime}-20^{\circ} 48^{\prime} \mathrm{E}\right)$ is located in the Balkan Peninsula (cf. Fig. 1a) and shared between Albania and the former Yugoslav Republic of Macedonia (FYROM). The lake is $30 \mathrm{~km}$ long and $15 \mathrm{~km}$ wide, covers an area of $358 \mathrm{~km}^{2}$, and is situated at an altitude of $693 \mathrm{~m}$ above sea level (ma.s.l.). The lake basin has a simple tublike shape with a volume of $55.4 \mathrm{~km}^{3}$ and a maximum water depth of $293 \mathrm{~m}$ (Lindhorst et al., 2015). The lake is oligotrophic today due to the large water volume and the low nutrient availability (Wagner et al., 2010) and has a specific conductivity of ca. $200 \mu \mathrm{S} \mathrm{cm}^{-1}$ and a pH of around 8.4 (Matter et al., 2010) in the surface waters. The hydrological characteristics are mainly controlled by a relatively low water input of $37.9 \mathrm{~m}^{3} \mathrm{~s}^{-1}$, of which $\sim 50 \%$ derives from karst aquifers (Matzinger et al., 2006a, b). The natural catchment area is relatively small with $1042 \mathrm{~km}^{2}$ (Matzinger et al., 2006b; Wagner et al., 2008). Lake Ohrid is situated within the Lake Ohrid basin, which is a $40 \mathrm{~km}$ long N-S trending graben structure in the Dinarides-Albanides-Hellenides mountain belt. The basin formation was initiated during a late phase of Alpine orogeny in the Miocene as a pull-apart basin in a short transtensional phase and consequently widened by an E-W extensional phase since the Pliocene (Lindhorst et al., 2015). The exact age of the formation of the lake is still unknown and the subject of several studies within the SCOPSCO project. 


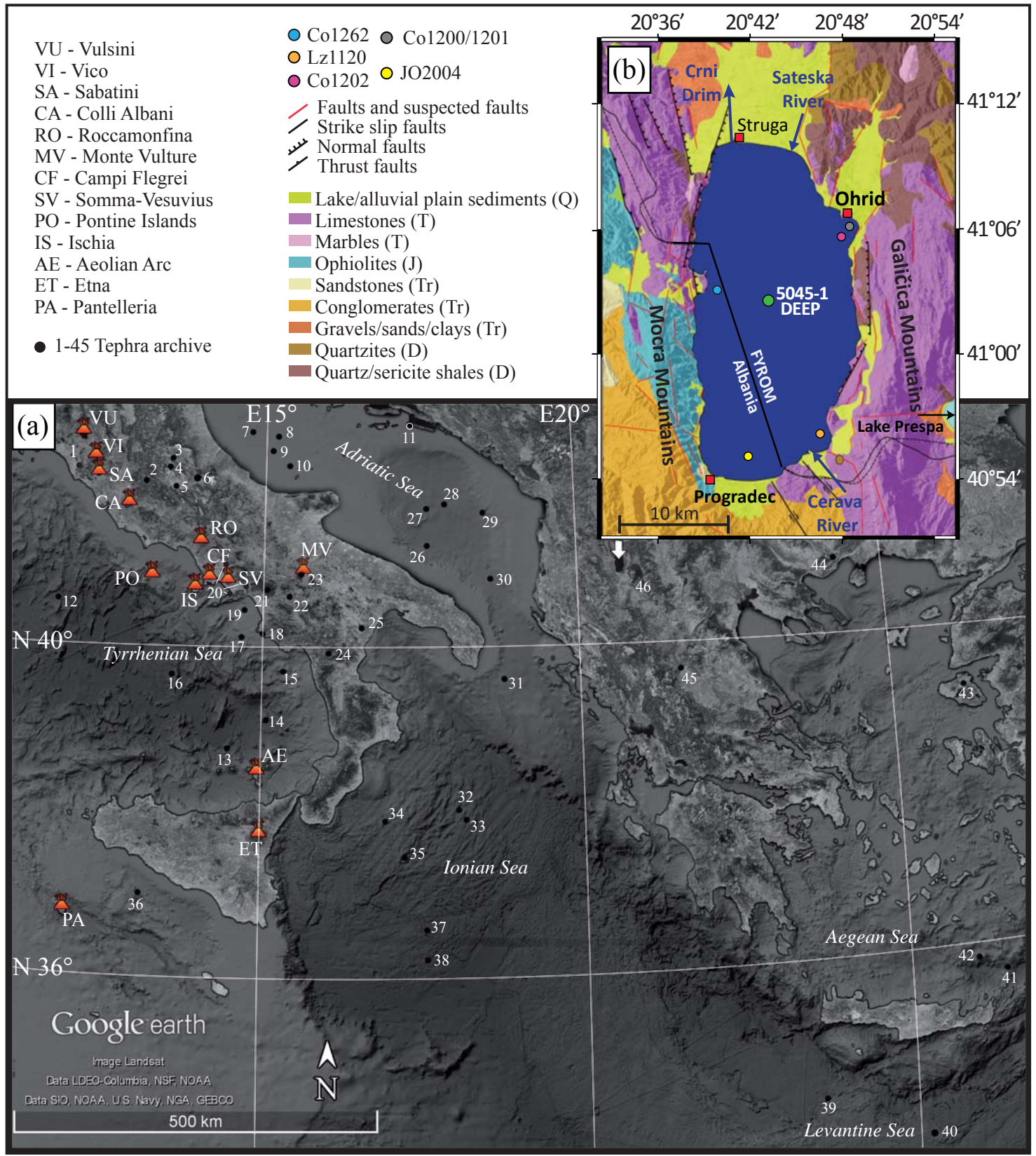

Figure 1. Panel (a): location of Lake Ohrid in the Mediterranean region, the Italian volcanoes, and the locations of archives mentioned in the text (1 - Ficoncella Site; 2 - Carsoli Basin; 3 - Paganica-San Demetrio-Castelnuovo Basin; 4 - Campo Felice; 5 - Fucino Basin; 6 - Sulmona Basin; 7 - PRAD1-2; 8 - CM92-42; 9 - RF93-77; 10 - RF95-11; 11 - Veliko Jezero, island of Mljet; 12 - KET8022; 13 - KET8003; 14 KET8011; 15 - C-45; 16 - KET8004-DED8708; 17 - C1202; 18 - Cilento coast; 19 - C106; 20 - SMP1e (CE1/CD1/SMP1); 21 - Acerno Basin; 22 - San Gregorio Magno Basin; 23 - Lago Grande di Monticchio; 24 - Mercure Basin; 25 - Montalbano Jonico succession; 26 MD90-917; 27 - KET8218; 28 - IN68-9; 29 - IN68-5; 30 - AD91-17; 31 - MD90-918; 32 - RC9 191; 33 - M25/4-12; 34 - KET8222; 35 - V10 69; 36 - ODP 963A; 37 - 22M-60; 38 - KC01B; 39 - RC9 183; 40 - RC9 181; 41-LC01; 42 - V10 58; 43 - Lesvos; 44 - Tenaghi Philippon; 45 - Theopetra Cave; 46 - Lake Prespa). Panel (b): geological map of the Lake Ohrid catchment modified after Lindhorst et al. (2015) and the core locations from previous studies (Lz1120, Co1200-1201-1202-1262, and JO2004) and the ICDP main drilling site DEEP.

In the $\mathrm{N}$ and $\mathrm{NE}$ of the basin, Palaeozoic metamorphic rocks crop out, on which Triassic to Early Jurassic karstified platform carbonates are superimposed (cf. Fig. 1b) in the E, NW and, in the SE, at the Galičica Mountains, up to $2300 \mathrm{~m}$ a.s.l. high (Robertson and Shallo, 2000). Jurassic ophiolites and Tertiary conglomerates form the Mocra 
Mountains of the SW part of the graben shoulder (Robertson and Shallo, 2000). Mesozoic intrusions of rhyolites and diabases are locally preserved in between the limestones and dolomites in the NE (Hoffmann et al., 2010). The plains in the $\mathrm{N}$ and $\mathrm{S}$ of Lake Ohrid are covered with lacustrine and alluvial plain sediments of Quaternary age (Watzin et al., 2002). The occurrence of a hydrothermal field near Kosel in the north of the Lake Ohrid basin is most likely fault-related since no indications of volcanic activity in younger times have been found during detailed field mapping (Reicherter et al., 2011).

\section{Materials and methods}

During the ICDP deep drilling campaign at Lake Ohrid in spring 2013, six parallel holes were drilled at the main drill site, the DEEP site (cf. Fig. 1b), down to a maximum sediment depth of $569 \mathrm{~m}$ below lake floor (m b.l.f.). The coring location ( $40 \mathrm{~m}$ distance between the individual holes) can be averaged to $41^{\circ} 02^{\prime} 57^{\prime \prime} \mathrm{N}$ and $20^{\circ} 42^{\prime} 54^{\prime \prime} \mathrm{E}$ with a water depth of $243 \mathrm{~m}$.

The cores of the DEEP site sequence were opened lengthwise, described visually, and subsequently scanned by Xray fluorescence (XRF) with an ITRAX core scanner $(\mathrm{Cr}$ tube, $30 \mathrm{~mA} 30 \mathrm{kV}$; COX Ltd, Sweden) at the University of Cologne. Based on the core description and the XRF data, the uppermost $247.8 \mathrm{mcd}$ of the DEEP site sequence was correlated to a composite profile (Francke et al., 2016). The core halves were visually screened for conspicuous horizons showing changes in macroscopic grain size or colour to identify potential tephra layers. Smear slides of these horizons were then checked for the occurrence of glass shards and micro-pumices using a Leitz DM EP polarization microscope. Once a tephra layer was identified, bulk samples were taken from the respective horizon and embedded in epoxy resin. The epoxy pucks were polished to avoid compositional variations due to topographic effects and carbon-coated to enable conductivity during the following scanning electron microscope (SEM) and energy dispersive spectroscopy (EDS) and wavelength dispersive spectroscopy (WDS) analysis. A first screening of major element compositions of single glass shards and micro-pumice was done using an SEM Philips XL30 equipped with an EDAX DX4 EDS system at the University of Pisa. Operating conditions were adjusted to $20 \mathrm{kV}$ accelerating energy, $200-500 \mathrm{~nm}$ beam diameter, 9-10 A beam current, 100 s live time with 2100-2400 shots per second, and ZAF correction (Z: atomic number; A: absorption; F: fluorescence). The initial calibration, using four reference standards (albite, olivine, and the glasses CFA47 and KE12), and the performance of the machine are described in detail by Marianelli and Sbrana (1998). In order to obtain more accurate quantitative analysis and to enlarge the comparability or reproducibility with existing data sets, a second screening of the samples was performed using the more common WDS technique at the Istituto di Geologia
Ambientale e Geoingegneria of the Italian National Research Council (IGAG-CNR, Rome). A Cameca SX50 electron microprobe equipped with a five-wavelength dispersive spectrometer was set to the following operating conditions: accelerating voltage, $15 \mathrm{kV}$; beam current, $15 \mathrm{nA}$; beam diameter, $10-15 \mu \mathrm{m}$; and counting time $20 \mathrm{~s}$ per element. Wollastonite ( $\mathrm{Si}$ and $\mathrm{Ca})$, corundum $(\mathrm{Al})$, diopside $(\mathrm{Mg})$, andradite $(\mathrm{Fe})$, rutile $(\mathrm{Ti})$, orthoclase $(\mathrm{K})$, jadeite $(\mathrm{Na})$, phlogopite $(\mathrm{F})$, potassium chloride $(\mathrm{Cl})$, baritina (S), and metals $(\mathrm{Mn})$ were used as standards (respective elements are given in brackets). Titanium contents were corrected for the overlap of the Ti and $\mathrm{K}$ peaks. Two international secondary standards (Kanui augite and rhyolite RLS132 glasses, from the United States Geological Survey) were analysed prior to sample measurements to evaluate the accuracy of the analyses. The mean analytical precision was $<1 \%$ for $\mathrm{SiO}_{2}$ and up to $1,5,15,30$, and $>50 \%$ for all the elements in concentration ranges of $15-30$, $5-15,1-5,1-0.3$, and $<0.3 \mathrm{wt} . \%$, respectively. All geochemical compositions were recalculated as being $100 \%$ waterfree. The geochemical classification of the composition of shards and micro-pumice was done with a total alkali vs. silica (TAS) diagram (Le Bas et al., 1986). Identified tephra layers are clearly labelled with the site name (OH-DP for Ohrid-DEEP) and the correlated bottom depth of each layer (e.g. "OH-DP-corr. [depth in dm]").

\section{Results and discussion}

The uppermost $247.8 \mathrm{mcd}$ of sediments from the DEEP site sequence mainly consists of fine-grained hemipelagic sediments with some intercalated coarse-grained beds, which were classified as event layers such as tephra and mass movement deposits. A detailed lithological description and the discussion of sedimentological processes and palaeoenvironmental information is given by Francke et al. (2016). Interglacial sediments indicate high amounts of calcite and organic matter, whereas glacial sediments are dominated by clastic, terrigenous components.

In total, 34 macroscopic tephra layers were recognized in the upper $247.8 \mathrm{mcd}$ of the DEEP site sequence (Fig. 2). Twelve of the 34 macroscopic tephra and 1 cryptotephra horizon have been unambiguously correlated with known eruptions of Italian volcanoes and represent the focus of the present paper. A detailed geochemical description and interpretation of the remaining tephra layers, which are not all analysed yet, and a discussion of potential ages and origins will be given elsewhere, as it is beyond the scope of this paper.

A local origin of the tephra layers from the direct surroundings of Lake Ohrid is highly unlikely, as there is no volcanic activity recorded for the Quaternary (cf. Fig. 1b). Potential volcanic sources within the Mediterranean area are the Italian provinces, the Hellenic Arc, or the Anatolian provinces. Pyroclastic deposits from the Hellenic Arc and the Anatolian provinces, mainly high-silica calc-alkaline tephra, 
were primarily dispersed in eastern directions (Druitt et al., 1995, 1999; Aksu et al., 2008; Hamann et al., 2010; Sulpizio et al., 2013; Sumita and Schmincke, 2013; Keller et al., 2014; Tomlinson et al., 2015) and so far have not been found in the sediment records of Lake Ohrid (Sulpizio et al., 2010) and nearby Lake Prespa (Damaschke et al., 2013). Furthermore, they were not found in the numerous studied tephrochronological successions from the Tyrrhenian, Adriatic, or western Ionian Sea.

A mainly eastern distribution is also assumed for Italian tephra layers, and some of them have even been found in the eastern Mediterranean and Aegean Sea (Margari et al., 2007; Sulpizio et al., 2010; Karkanas et al., 2015; Satow et al., 2015). All tephra layers discussed here show an alkaline affinity, which, in the Mediterranean region, is only known from the Italian volcanic provinces (Peccerillo, 2005). The existing records from lakes Ohrid and Prespa only contain tephra from Italian volcanoes, which was transported by the prevailing westerlies with $\mathrm{E}-\mathrm{SE}$ directions in the upperatmospheric wind system. The Quaternary Italian volcanism is divided into several magmatic provinces (Washington, 1906; Peccerillo, 2005), which are mainly located along the Tyrrhenian coast of the Italian Peninsula, in the Tyrrhenian Sea, and in the Sicilian Channel, between 500 and $1000 \mathrm{~km}$ away from Lake Ohrid (Fig. 1a). In the studied succession ( $<637 \mathrm{kyr}$ ) explosive volcanic activity of Italian volcanoes is known so far from Roman province (Vulsini, Vico, Sabatini, Colli Albani), the Ernici-Roccamonfina province (Monti Ernici, Roccamonfina), the Campania province (SommaVesuvius, Campi Flegrei, Procida, Ischia), the Pontine Islands (Ventotene, Santo Stefano), the Monte Vulture, the Aeolian Arc (Alicudi, Filicudi, Salina, Lipari, Vulcano, Panarea, Stromboli), and the Sicily province (Mt. Etna, Ustica, Pantelleria; cf. Fig. 1a; Peccerillo, 2005). The products of these Italian volcanoes span a wide compositional field ranging from subalkaline to alkaline and from mafic to silicic, depending on their geodynamic setting or mantle source (Lustrino et al., 2011). Most of them have a potassium or a high potassium affinity (Rome, Roccamonfina, Campania, and Pontine Islands), and their large chemical similarity or overlap in composition makes tephrostratigraphic work very challenging (Sulpizio et al., 2010). An origin in one of these provinces is suggested by the (high) alkaline phonolitic and trachytic compositions of most of the tephra layers found in the DEEP site sequence. However, some of the tephra layers in the DEEP site succession show more specific and unique compositions, which allow more straightforward correlations to Pantelleria and Somma-Vesuvius (Peccerillo, 2005) or the silica-undersaturated, poorly evolved pyroclastic products of the Colli Albani (Giaccio et al., 2013a).

\subsection{OH-DP-0027-Mercato}

Cryptotephra OH-DP-0027 was recognized by correlating the patterns of the XRF potassium curve of the DEEP site

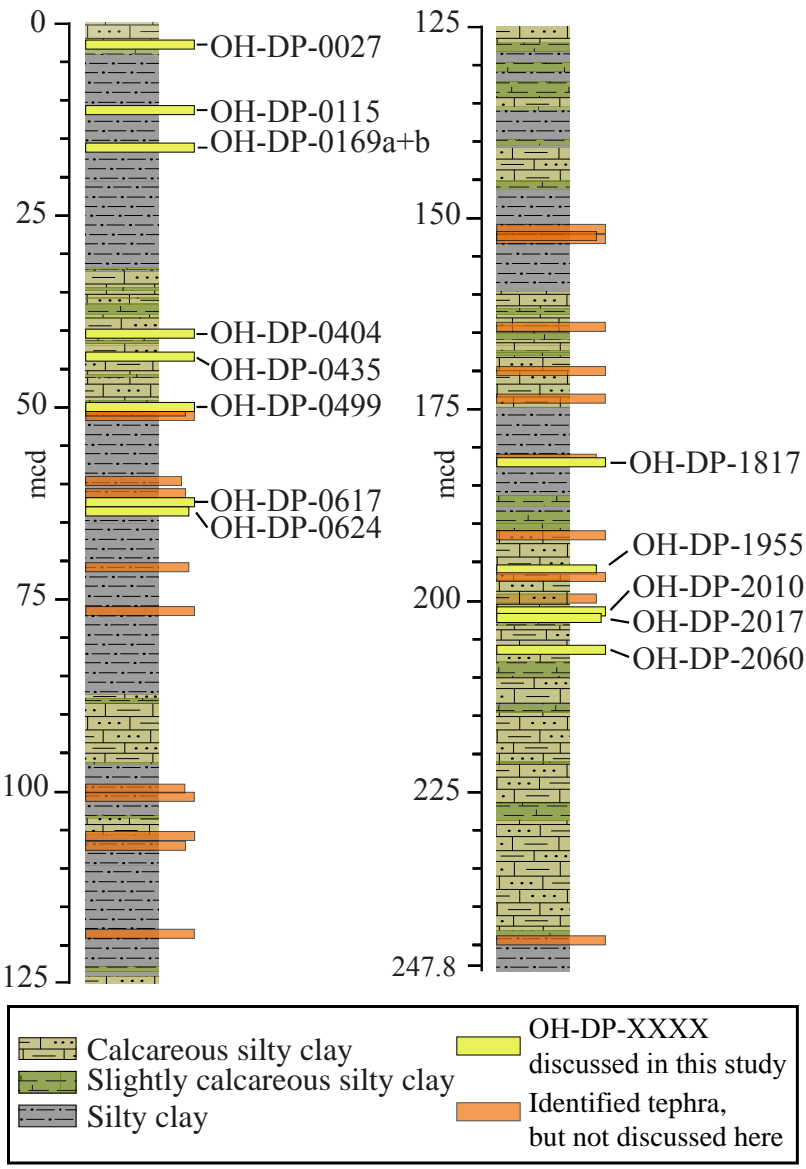

Figure 2. (Litho-) Stratigraphy of the uppermost $247.8 \mathrm{mcd}$ of the DEEP site sequence and the position of recognized tephra layers. Tephra layers coloured in yellow and labelled are discussed in this paper. The thickness of tephra layers is not to scale and more detailed information about tephra thickness and appearance is provided in the text.

record with those of the nearby core Co1262 (cf. Fig. 1; Wagner et al., 2012). In both cores, a potassium peak is observed in the same stratigraphic position, which in core Co1262 is caused by the presence of glass shards of cryptotephra Co1262-709. In the DEEP site sequence, the corresponding peak culminates at about $2.775 \mathrm{mcd}$. A microscopic inspection of the interval $2.770-2.780 \mathrm{mcd}$ revealed the occurrence of glass shards. Since cryptotephra is dispersed in the sediment and does not form a continuous distinct horizon, the exact depth of the tephra isochron was set to the maximum of the $K$ peak $(2.775 \mathrm{mcd})$, most likely representing the maximum shard concentration.

The discovered volcanic particles of OH-DP-0027 are highly vesicular micro-pumices with spherical or elongated vesicles and glass shards with thick septa or a platy shape. The composition of OH-DP-0027 is Na-phonolitic (Table 1, Fig. 2a) and thus similar to cryptotephra Co1262-709 (Wagner et al., 2012) and the associated cryptotephra horizons 
Table 1. Average major element glass composition (normalized to 100\%) of investigated tephra layers OH-DP-0027 to OH-DP-2060 of the DEEP site sequence, the correlations discussed, and the standards used for evaluation of the accuracy of the analyses. All data of the Ohrid tephra layers were obtained using an electron microprobe outfitted with WDS. The total is given as analytical total, and the tephra layers printed in italics are not correlated with the respective Ohrid tephra and are discussed only. The full data set of OH-DP-0027 to OH-DP-2060 is given in Table S1 in the Supplement, and a list of all references used can be found in Table A1 in the Appendix. $\overline{\mathrm{x}}$ : mean; SD: standard deviation; NA: not available; TBA: Tufo di Bagni Albule.

\begin{tabular}{|c|c|c|c|c|c|c|c|c|c|c|c|c|c|c|c|c|c|}
\hline & & $\mathrm{SiO}_{2}$ & $\mathrm{TiO}_{2}$ & $\mathrm{Al}_{2} \mathrm{O}_{3}$ & $\mathrm{FeO}_{\mathrm{TOT}}$ & $\mathrm{MnO}$ & $\mathrm{MgO}$ & $\mathrm{CaO}$ & $\mathrm{Na}_{2} \mathrm{O}$ & $\mathrm{K}_{2} \mathrm{O}$ & $\mathrm{P}_{2} \mathrm{O}_{5}$ & $\mathrm{~F}$ & $\mathrm{Cl}$ & $\mathrm{SO}_{3}$ & Total & Tot. alkali & Alk. ratio \\
\hline OH-DP-0027 & $\overline{\mathrm{x}}$ & 59.29 & 0.17 & 20.70 & 2.19 & 0.18 & 0.19 & 2.33 & 8.10 & 6.81 & 0.03 & 0.67 & 0.59 & 0.06 & 97.49 & 14.91 & 0.84 \\
\hline$n=15$ & SD & 1.12 & 0.06 & 0.88 & 0.94 & 0.07 & 0.37 & 1.31 & 0.38 & 0.63 & 0.02 & 0.13 & 0.06 & 0.03 & 1.75 & 0.91 & 0.06 \\
\hline OT0702-3 & $\overline{\mathrm{x}}$ & 59.10 & 0.17 & 21.58 & 1.95 & 0.17 & 0.16 & 1.76 & 7.56 & 7.02 & - & - & 0.52 & - & 100.00 & 14.58 & 0.93 \\
\hline$n=9$ & SD & 0.51 & 0.08 & 0.12 & 0.11 & 0.06 & 0.09 & 0.13 & 0.53 & 0.26 & - & - & 0.03 & - & - & 0.66 & 0.07 \\
\hline Co1262-709 & $\overline{\mathrm{x}}$ & 58.59 & 0.17 & 21.81 & 1.84 & 0.16 & 0.12 & 1.67 & 8.20 & 6.94 & 0.00 & - & 0.51 & - & 100 & 15.14 & 0.85 \\
\hline$n=12$ & SD & 0.39 & 0.08 & 0.49 & 0.12 & 0.06 & 0.08 & 0.22 & 0.50 & 0.29 & 0.00 & - & 0.03 & - & - & 0.36 & 0.08 \\
\hline РT0915-2 & $\overline{\mathrm{x}}$ & 58.34 & 0.18 & 21.59 & 1.92 & 0.15 & 0.24 & 1.97 & 7.93 & 6.85 & 0.09 & - & 0.73 & - & 100.00 & 14.79 & 0.87 \\
\hline$n=10$ & SD & 0.30 & 0.05 & 0.38 & 0.12 & 0.06 & 0.10 & 0.42 & 0.24 & 0.18 & 0.18 & - & 0.06 & - & - & 0.33 & 0.03 \\
\hline тмба & $\overline{\mathrm{x}}$ & 59.98 & 0.30 & 20.61 & 2.24 & 0.13 & 0.16 & 2.42 & 5.58 & 8.02 & 0.08 & 0.08 & 0.51 & - & 97.67 & 13.60 & 1.51 \\
\hline$n=13$ & SD & 0.93 & 0.09 & 0.51 & 0.46 & 0.04 & 0.07 & 0.39 & 1.03 & 0.82 & 0.12 & 0.00 & 0.15 & - & 0.91 & 0.57 & 0.38 \\
\hline TM6b & $\overline{\mathrm{x}}$ & 58.71 & 0.16 & 21.10 & 1.99 & 0.19 & 0.15 & 2.11 & 8.14 & 6.82 & 0.13 & 0.10 & 0.49 & - & 98.21 & 14.96 & 0.88 \\
\hline$n=12$ & SD & 1.53 & 0.09 & 0.83 & 1.00 & 0.07 & 0.26 & 1.19 & 1.11 & 0.88 & 0.20 & 0.09 & 0.18 & - & 1.27 & 0.97 & 0.31 \\
\hline MJ1-VJ1 & $\overline{\mathrm{x}}$ & 58.76 & 0.21 & 21.03 & 1.85 & 0.19 & 0.12 & 2.41 & 7.38 & 7.12 & - & 0.30 & 0.57 & 0.06 & 98.12 & 14.51 & 0.96 \\
\hline$n=27$ & SD & 1.42 & 0.11 & 0.81 & 0.81 & 0.08 & 0.21 & 1.24 & 0.73 & 0.85 & - & 0.18 & 0.21 & 0.04 & 0.88 & 1.51 & 0.07 \\
\hline KET8218 V1 & $\overline{\mathrm{x}}$ & 60.04 & 0.10 & 21.49 & 1.74 & 0.00 & 0.00 & 1.82 & 7.72 & 7.05 & - & - & & & 99.96 & 14.77 & 0.91 \\
\hline$n=\mathrm{NA}$ & SD & - & - & - & - & - & - & - & - & - & - & - & - & - & - & - & - \\
\hline IN68-9 $125 \mathrm{~cm}$ & $\overline{\mathrm{x}}$ & 59.50 & 0.09 & 21.26 & 1.71 & 0.11 & 0.14 & 1.81 & 7.72 & 7.14 & - & - & 0.52 & - & 93.40 & 14.86 & 0.92 \\
\hline$n=20$ & SD & 0.78 & 0.02 & 0.34 & 0.17 & 0.06 & 0.12 & 0.43 & 0.57 & 0.62 & - & - & 0.08 & - & 3.53 & 0.80 & 0.11 \\
\hline IN68-5 $259 \mathrm{~cm}$ & $\overline{\mathrm{x}}$ & 58.81 & 0.09 & 21.21 & 1.79 & 0.12 & 0.10 & 1.75 & 8.18 & 7.40 & - & - & 0.55 & - & 95.54 & 15.58 & 0.91 \\
\hline$n=15$ & SD & 0.36 & 0.03 & 0.15 & 0.10 & 0.06 & 0.07 & 0.09 & 0.37 & 0.13 & - & - & 0.04 & - & 1.82 & 0.33 & 0.05 \\
\hline RF95-11 $320 \mathrm{~cm}$ & $\overline{\mathrm{x}}$ & 59.10 & 0.11 & 20.60 & 1.89 & 0.13 & 0.08 & 1.88 & 7.58 & 8.13 & - & - & 0.50 & - & 95.94 & 15.71 & 1.08 \\
\hline$n=21$ & SD & 0.76 & 0.05 & 0.26 & 0.30 & 0.06 & 0.05 & 0.36 & 0.67 & 0.45 & - & - & 0.12 & - & 1.64 & 0.84 & 0.11 \\
\hline RF95-11 $320 \mathrm{~cm}$ & $\overline{\mathrm{x}}$ & 61.43 & 0.27 & 18.46 & 2.95 & 0.12 & 0.32 & 1.97 & 5.29 & 8.52 & - & - & 0.67 & - & 94.95 & 13.81 & 1.65 \\
\hline$n=3$ & SD & 0.56 & 0.06 & 0.09 & 0.24 & 0.07 & 0.28 & 0.19 & 0.83 & 0.94 & - & - & 0.21 & - & 1.18 & 0.40 & 0.24 \\
\hline MD90-918 $210 \mathrm{~cm}$ & $\overline{\mathrm{x}}$ & 58.71 & 0.14 & 21.82 & 1.78 & 0.17 & 0.17 & 1.74 & 8.06 & 6.80 & - & - & 0.60 & - & - & 14.86 & 0.85 \\
\hline$n=9$ & SD & 0.47 & 0.08 & 0.23 & 0.11 & 0.10 & 0.06 & 0.16 & 0.35 & 0.15 & - & - & 0.11 & - & - & 0.38 & 0.04 \\
\hline MD90-918 $223 \mathrm{~cm}$ & $\overline{\mathrm{x}}$ & 58.82 & 0.12 & 21.88 & 1.72 & 0.13 & 0.11 & 1.81 & 8.27 & 6.60 & - & - & 0.53 & - & 100.00 & 14.88 & 0.80 \\
\hline$n=12$ & SD & 0.61 & 0.10 & 0.28 & 0.15 & 0.12 & 0.06 & 0.53 & 0.37 & 0.81 & - & - & 0.09 & - & - & 1.00 & 0.10 \\
\hline AD94-17 190-191 cm & $\overline{\mathrm{x}}$ & 58.69 & 0.14 & 21.97 & 1.70 & 0.14 & 0.26 & 1.72 & 8.17 & 6.73 & - & - & 0.49 & - & 100.00 & 14.90 & 0.82 \\
\hline$n=14$ & $\mathrm{SD}$ & 0.74 & 0.11 & 0.21 & 0.16 & 0.09 & 0.09 & 0.34 & 0.43 & 0.47 & - & - & 0.09 & - & - & 0.79 & 0.05 \\
\hline AD94-17 $195-196 \mathrm{~cm}$ & $\overline{\mathrm{x}}$ & 58.51 & 0.13 & 22.13 & 1.73 & 0.15 & 0.43 & 1.68 & 8.13 & 6.65 & - & - & 0.51 & - & 100.00 & 14.78 & 0.83 \\
\hline$n=10$ & SD & 0.31 & 0.07 & 0.32 & 0.15 & 0.09 & 0.09 & 0.21 & 0.79 & 0.45 & - & - & 0.06 & - & - & 0.38 & 0.15 \\
\hline OH-DP-0115 & $\overline{\mathrm{x}}$ & 61.60 & 0.38 & 18.24 & 3.43 & 0.12 & 0.70 & 2.67 & 3.43 & 9.32 & 0.10 & 0.14 & 0.46 & 0.11 & 95.49 & 12.76 & 2.77 \\
\hline$n=16$ & SD & 0.89 & 0.03 & 0.17 & 0.32 & 0.05 & 0.16 & 0.32 & 0.44 & 0.44 & 0.04 & 0.09 & 0.10 & 0.06 & 1.98 & 0.15 & 0.45 \\
\hline ОТ0702-4 & $\overline{\mathrm{x}}$ & 61.27 & 0.39 & 18.70 & 3.11 & 0.11 & 0.67 & 2.35 & 3.73 & 9.22 & - & - & 0.46 & - & 100.00 & 12.96 & 2.52 \\
\hline$n=12$ & $\mathrm{SD}$ & 0.61 & 0.05 & 0.12 & 0.20 & 0.06 & 0.17 & 0.24 & 0.44 & 0.46 & - & - & 0.10 & - & - & 0.11 & 0.42 \\
\hline OT0520-2 & $\overline{\mathrm{x}}$ & 61.88 & 0.29 & 18.63 & 2.92 & 0.03 & 0.50 & 2.20 & 4.18 & 8.82 & - & - & 0.54 & - & 100.00 & 12.99 & 2.15 \\
\hline$n=12$ & SD & 0.50 & 0.08 & 0.18 & 0.22 & 0.06 & 0.15 & 0.18 & 0.45 & 0.49 & - & - & 0.13 & - & - & 0.09 & 0.35 \\
\hline JO187 & $\overline{\mathrm{x}}$ & 60.99 & 0.40 & 18.74 & 3.26 & 0.11 & 0.68 & 2.38 & 3.59 & 9.43 & - & - & 0.42 & - & 100.00 & 13.02 & 2.68 \\
\hline$n=10$ & SD & 0.71 & 0.10 & 0.10 & 0.26 & 0.07 & 0.15 & 0.25 & 0.48 & 0.41 & - & - & 0.09 & - & - & 0.28 & 0.46 \\
\hline ОТ0700-1 & $\overline{\mathrm{x}}$ & 61.60 & 0.38 & 18.74 & 3.03 & 0.10 & 0.67 & 2.30 & 3.74 & 8.94 & - & - & 0.50 & - & 100.00 & 12.68 & 2.44 \\
\hline$n=12$ & SD & 0.75 & 0.10 & 0.15 & 0.34 & 0.09 & 0.16 & 0.30 & 0.44 & 0.43 & - & - & 0.13 & - & - & 0.24 & 0.39 \\
\hline РТ0915-5 & $\overline{\mathrm{x}}$ & 60.85 & 0.40 & 18.92 & 3.40 & 0.11 & 0.79 & 2.37 & 3.60 & 8.94 & 0.01 & - & 0.62 & - & 100.00 & 12.54 & 2.57 \\
\hline$n=12$ & SD & 0.87 & 0.08 & 0.27 & 0.37 & 0.07 & 0.19 & 0.30 & 0.58 & 0.56 & 0.03 & - & 0.19 & - & - & 0.27 & 0.56 \\
\hline M25/4-12 Y-3 & $\overline{\mathrm{x}}$ & 61.32 & 0.37 & 18.27 & 3.23 & 0.12 & 0.62 & 2.42 & 3.64 & 9.41 & 0.11 & - & 0.50 & - & 96.22 & 13.04 & 2.69 \\
\hline$n=33$ & SD & 0.89 & 0.03 & 0.26 & 0.34 & 0.05 & 0.19 & 0.26 & 0.62 & 0.69 & 0.04 & - & 0.18 & - & 1.35 & 0.17 & 0.61 \\
\hline RC9 191 Y-3 & $\overline{\mathrm{x}}$ & 62.76 & 0.36 & 18.21 & 2.98 & 0.17 & 0.25 & 2.07 & 4.44 & 8.75 & - & - & - & - & - & - & - \\
\hline$n=\mathrm{NA}$ & SD & 0.36 & 0.03 & 0.16 & 0.21 & 0.12 & 0.12 & 0.15 & 0.19 & 0.26 & - & - & - & - & - & - & - \\
\hline TM15 & $\overline{\mathrm{x}}$ & 62.22 & 0.38 & 18.36 & 3.27 & 0.13 & 0.61 & 2.19 & 3.85 & 8.36 & 0.12 & - & 0.52 & - & 96.32 & 12.21 & 2.21 \\
\hline$n=20$ & SD & 0.76 & 0.03 & 0.20 & 0.29 & 0.04 & 0.14 & 0.22 & 0.43 & 0.54 & 0.06 & - & 0.10 & - & 0.46 & 0.21 & 0.36 \\
\hline TP-9.70 & $\overline{\mathrm{x}}$ & 61.79 & 0.37 & 18.28 & 3.17 & 0.10 & 0.59 & 2.40 & 3.78 & 9.19 & 0.12 & - & 0.54 & - & 96.46 & 12.97 & 2.48 \\
\hline$n=42$ & SD & 0.78 & 0.04 & 0.17 & 0.31 & 0.04 & 0.14 & 0.22 & 0.47 & 0.47 & 0.04 & - & 0.12 & - & 1.42 & 0.19 & 0.41 \\
\hline S19 & $\overline{\mathrm{x}}$ & 62.17 & 0.31 & 18.77 & 2.96 & 0.08 & 0.49 & 2.13 & 4.20 & 8.90 & - & - & - & - & 100.01 & 13.10 & 2.12 \\
\hline$n=\mathrm{NA}$ & SD & 1.15 & 0.07 & 0.14 & 0.47 & 0.04 & 0.26 & 0.29 & 0.38 & 0.50 & - & - & - & - & - & - & - \\
\hline $\mathrm{C} 106 \mathrm{~A} 2$ & $\overline{\mathrm{x}}$ & 62.63 & 0.34 & 18.24 & 3.15 & 0.15 & 0.47 & 2.12 & 4.15 & 8.75 & - & - & - & - & 100.00 & 12.90 & 2.11 \\
\hline$n=12$ & SD & 0.20 & 0.06 & 0.12 & 0.12 & 0.08 & 0.08 & 0.10 & 0.19 & 0.14 & - & - & - & - & - & - & - \\
\hline C45 B2 & $\overline{\mathrm{x}}$ & 62.41 & 0.33 & 18.30 & 3.17 & 0.15 & 0.50 & 2.13 & 4.30 & 8.71 & - & - & - & - & 100.00 & 13.01 & 2.03 \\
\hline$n=11$ & SD & 0.32 & 0.10 & 0.29 & 0.10 & 0.08 & 0.09 & 0.10 & 0.15 & 0.08 & - & - & - & - & - & - & - \\
\hline MD90-917 920-17 & $\overline{\mathrm{x}}$ & 61.41 & 0.37 & 18.72 & 3.17 & 0.08 & 0.70 & 2.44 & 3.52 & 9.14 & - & - & 0.44 & - & 100.00 & 12.66 & 2.60 \\
\hline$n=\mathrm{NA}$ & SD & 0.86 & 0.10 & 0.17 & 0.38 & 0.08 & 0.21 & 0.33 & 0.40 & 0.40 & - & - & 0.09 & - & - & - & - \\
\hline KET8004 C-7 & $\overline{\mathrm{x}}$ & 63.12 & 0.25 & 18.53 & 3.03 & 0.00 & 0.14 & 2.17 & 3.86 & 8.86 & - & - & - & - & 99.96 & 12.72 & 2.30 \\
\hline$n=\mathrm{NA}$ & SD & 0.90 & 0.08 & 0.21 & 0.39 & 0.00 & 0.13 & 0.33 & 0.52 & 0.42 & - & - & - & - & - & - & - \\
\hline KET8011 C-7 & $\overline{\mathrm{x}}$ & 61.90 & 0.33 & 19.14 & 2.95 & 0.00 & 0.49 & 2.75 & 3.62 & 8.79 & - & - & - & - & 99.97 & 12.41 & 2.43 \\
\hline$n=\mathrm{NA}$ & SD & - & - & - & - & - & - & - & - & - & - & - & - & - & - & - & - \\
\hline
\end{tabular}


Table 1. Continued.

\begin{tabular}{|c|c|c|c|c|c|c|c|c|c|c|c|c|c|c|c|c|c|}
\hline & & $\mathrm{SiO}_{2}$ & $\mathrm{TiO}_{2}$ & $\mathrm{Al}_{2} \mathrm{O}_{3}$ & $\mathrm{FeO}_{\mathrm{TOT}}$ & $\mathrm{MnO}$ & $\mathrm{MgO}$ & $\mathrm{CaO}$ & $\mathrm{Na}_{2} \mathrm{O}$ & $\mathrm{K}_{2} \mathrm{O}$ & $\mathrm{P}_{2} \mathrm{O}_{5}$ & $\mathrm{~F}$ & $\mathrm{Cl}$ & $\mathrm{SO}_{3}$ & Total & Tot. alkali & Alk. ratio \\
\hline OH-DP-0169b & $\overline{\mathrm{x}}$ & 61.76 & 0.43 & 18.86 & 3.01 & 0.25 & 0.36 & 1.86 & 5.96 & 7.47 & 0.04 & 0.33 & 0.83 & 0.05 & 97.04 & 13.43 & 1.26 \\
\hline$n=18$ & SD & 0.29 & 0.03 & 0.15 & 0.09 & 0.03 & 0.02 & 0.09 & 0.28 & 0.19 & 0.02 & 0.07 & 0.07 & 0.02 & 1.25 & 0.29 & 0.08 \\
\hline OH-DP-0169a & $\overline{\mathrm{x}}$ & 61.69 & 0.41 & 18.89 & 3.04 & 0.26 & 0.34 & 1.78 & 6.28 & 7.28 & 0.04 & 0.36 & 0.85 & 0.05 & 98.78 & 13.56 & 1.17 \\
\hline$n=25$ & SD & 0.44 & 0.03 & 0.14 & 0.09 & 0.04 & 0.02 & 0.04 & 0.56 & 0.15 & 0.02 & 0.07 & 0.04 & 0.02 & 1.56 & 0.57 & 0.17 \\
\hline OT0700-2 & $\overline{\mathrm{x}}$ & 60.84 & 0.39 & 19.21 & 2.98 & 0.20 & 0.42 & 1.77 & 6.06 & 7.45 & - & - & 0.68 & - & 100.00 & 13.51 & 1.29 \\
\hline$n=17$ & SD & 0.38 & 0.09 & 0.18 & 0.19 & 0.07 & 0.12 & 0.25 & 0.82 & 0.72 & - & - & 0.11 & - & - & 0.33 & 0.50 \\
\hline ОТ05020-3 & $\overline{\mathrm{x}}$ & 61.88 & 0.29 & 18.63 & 2.92 & 0.03 & 0.50 & 2.20 & 4.18 & 8.82 & - & - & 0.54 & - & 100.00 & 12.99 & 2.15 \\
\hline$n=12$ & SD & 0.50 & 0.08 & 0.18 & 0.22 & 0.06 & 0.15 & 0.18 & 0.45 & 0.49 & - & - & 0.13 & - & - & 0.09 & 0.37 \\
\hline ОТ0702-6 & $\overline{\mathrm{x}}$ & 60.83 & 0.40 & 19.13 & 2.93 & 0.18 & 0.48 & 1.77 & 6.31 & 7.34 & - & - & 0.65 & - & 100.00 & 13.65 & 1.17 \\
\hline$n=11$ & SD & 0.30 & 0.10 & 0.11 & 0.13 & 0.09 & 0.08 & 0.20 & 0.44 & 0.25 & - & - & 0.10 & - & - & 0.31 & 0.13 \\
\hline JO-244 & $\overline{\mathrm{x}}$ & 60.64 & 0.42 & 19.18 & 2.96 & 0.21 & 0.40 & 1.81 & 6.22 & 7.48 & - & - & 0.69 & - & 100.00 & 13.69 & 1.21 \\
\hline$n=18$ & SD & 0.57 & 0.08 & 0.24 & 0.16 & 0.05 & 0.12 & 0.21 & 0.36 & 0.31 & - & - & 0.10 & - & - & 0.20 & 0.13 \\
\hline OT0701-1/5 & $\overline{\mathrm{x}}$ & 60.92 & 0.41 & 19.15 & 3.02 & 0.20 & 0.44 & 1.80 & 5.87 & 7.57 & - & - & 0.64 & - & 100.00 & 13.44 & 1.37 \\
\hline$n=41$ & SD & 0.64 & 0.07 & 0.69 & 0.18 & 0.07 & 0.14 & 0.30 & 1.12 & 0.97 & - & - & 0.14 & - & - & 0.38 & 0.69 \\
\hline РT0704-3 & $\overline{\mathrm{x}}$ & 60.83 & 0.42 & 19.14 & 3.03 & 0.23 & 0.47 & 1.77 & 5.86 & 7.59 & - & - & 0.65 & - & 100.00 & 13.46 & 1.53 \\
\hline$n=105$ & SD & 0.58 & 0.09 & 0.23 & 0.26 & 0.09 & 0.16 & 0.32 & 1.01 & 0.82 & - & - & 0.13 & - & - & 0.42 & 0.69 \\
\hline RC9 191 Y-5 & $\overline{\mathrm{x}}$ & 61.56 & 0.44 & 18.90 & 3.59 & 0.17 & 0.59 & 1.97 & 5.55 & 7.26 & - & - & - & - & 96.48 & 12.80 & 1.31 \\
\hline V10 58 Y-5 & $x$ & 62.46 & 0.36 & 18.43 & 2.11 & 0.16 & 0.40 & 2.16 & 6.33 & 7.59 & - & - & - & - & 93.52 & 13.92 & 1.20 \\
\hline $\mathrm{C}-13$ & $\overline{\mathrm{x}}$ & 62.20 & 0.35 & 19.30 & 2.94 & 0.08 & 1.75 & 5.92 & 7.41 & - & - & - & - & - & 99.95 & 13.33 & 1.25 \\
\hline C-13 & $x$ & 61.50 & 0.31 & 19.15 & 3.13 & 0.33 & 2.50 & 3.54 & 9.50 & - & - & - & - & - & 99.96 & 13.04 & 2.68 \\
\hline PRAD1653 & $\overline{\mathrm{x}}$ & 61.00 & 0.43 & 19.71 & 2.93 & 0.24 & 0.28 & 1.68 & 6.72 & 7.01 & - & - & - & - & 95.89 & 13.73 & 1.04 \\
\hline$n=14$ & SD & 0.49 & 0.04 & 0.11 & 0.29 & 0.04 & 0.03 & 0.07 & 0.20 & 0.12 & - & - & - & - & 0.87 & 0.27 & 0.03 \\
\hline $\mathrm{I}-3$ & $\overline{\mathrm{x}}$ & 61.45 & 0.41 & 18.40 & 3.12 & 0.19 & 0.54 & 2.16 & 4.88 & 7.84 & 0.09 & 0.26 & 0.65 & - & 97.01 & 12.84 & 1.72 \\
\hline$n=14$ & SD & 1.14 & 0.05 & 0.43 & 0.43 & 0.06 & 0.24 & 0.56 & 0.94 & 0.97 & 0.06 & 0.11 & 0.21 & - & 2.48 & 0.47 & 0.63 \\
\hline Lc 214.925 & $\overline{\mathrm{x}}$ & 61.93 & 0.46 & 18.03 & 3.20 & 0.21 & 0.44 & 2.05 & 6.20 & 7.46 & - & - & - & - & 95.35 & 13.66 & 1.25 \\
\hline$n=32$ & SD & 0.74 & 0.10 & 0.25 & 0.47 & 0.08 & 0.36 & 0.70 & 0.94 & 0.48 & - & - & - & - & 1.33 & 0.93 & 0.30 \\
\hline TM18 & $\overline{\mathrm{x}}$ & 61.60 & 0.43 & 18.92 & 2.93 & 0.24 & 0.35 & 1.75 & 5.78 & 7.04 & 0.05 & 0.33 & 0.79 & - & 98.64 & 12.82 & 1.24 \\
\hline$n=61$ & SD & 0.46 & 0.03 & 0.36 & 0.11 & 0.03 & 0.07 & 0.16 & 0.75 & 0.53 & 0.02 & 0.04 & 0.08 & - & 1.71 & 0.83 & 0.25 \\
\hline ML2 & $\overline{\mathrm{x}}$ & 61.01 & 0.48 & 18.18 & 3.02 & 0.21 & 0.44 & 2.12 & 5.64 & 8.05 & - & 0.07 & 0.71 & - & 96.51 & 13.70 & 1.57 \\
\hline$n=105$ & SD & 0.42 & 0.04 & 0.19 & 0.21 & 0.06 & 0.15 & 0.32 & 1.20 & 1.02 & - & 0.04 & 0.20 & - & 1.46 & 0.32 & 0.69 \\
\hline S17 & $\overline{\mathrm{x}}$ & 61.67 & 0.33 & 19.18 & 2.90 & 0.22 & 0.27 & 1.60 & 6.42 & 7.38 & - & - & - & - & 100.00 & 13.80 & 1.15 \\
\hline$n=\mathrm{NA}$ & SD & 0.22 & 0.14 & 0.10 & 0.10 & 0.10 & 0.08 & 0.11 & 0.30 & 0.20 & - & - & - & - & - & 0.50 & - \\
\hline Tenaghi Philippon CI & $\overline{\mathrm{x}}$ & 63.29 & 0.40 & 19.42 & 3.32 & 0.20 & 0.45 & 2.09 & 5.20 & 8.48 & 0.10 & - & - & - & 96.42 & 13.69 & 1.99 \\
\hline$n=3$ & SD & 0.39 & 0.03 & 0.28 & 0.26 & 0.06 & 0.20 & 0.47 & 1.69 & 1.27 & 0.06 & - & - & - & 0.88 & 0.46 & 1.13 \\
\hline pre CI deposits & $\overline{\mathrm{x}}$ & 58.94 & 0.40 & 19.75 & 3.45 & 0.25 & 0.34 & 2.04 & 6.46 & 7.58 & - & - & 0.79 & - & 97.76 & 14.04 & 1.19 \\
\hline$n=68$ & SD & 0.38 & 0.02 & 0.16 & 0.14 & 0.03 & 0.04 & 0.10 & 0.51 & 0.30 & - & - & 0.04 & - & 1.32 & 0.26 & 0.14 \\
\hline OH-DP-0404 & $\overline{\mathrm{x}}$ & 57.49 & 0.54 & 19.05 & 5.10 & 0.17 & 1.30 & 4.33 & 4.06 & 7.72 & 0.24 & 0.18 & 0.50 & 0.09 & 97.08 & 11.78 & 1.93 \\
\hline$n=20$ & SD & 1.00 & 0.11 & 0.45 & 0.67 & 0.04 & 0.24 & 0.65 & 0.54 & 0.62 & 0.07 & 0.08 & 0.09 & 0.05 & 1.33 & 0.93 & 0.25 \\
\hline OT0702-8 & $\overline{\mathrm{x}}$ & 57.60 & 0.55 & 19.49 & 4.48 & 0.16 & 1.22 & 3.77 & 4.18 & 8.09 & 0.01 & - & 0.46 & - & 100.00 & 12.26 & 1.94 \\
\hline$n=12$ & SD & 0.46 & 0.08 & 0.15 & 0.33 & 0.07 & 0.15 & 0.32 & 0.18 & 0.43 & 0.02 & - & 0.05 & - & - & 0.43 & 0.15 \\
\hline TM24a & $\overline{\mathrm{x}}$ & 57.64 & 0.49 & 18.99 & 4.26 & 0.14 & 1.12 & 3.99 & 4.24 & 8.37 & 0.24 & - & 0.52 & - & 100.00 & 12.61 & 1.97 \\
\hline$n=35$ & SD & 1.14 & 0.06 & 0.19 & 0.46 & 0.02 & 0.22 & 0.56 & 0.14 & 0.31 & 0.06 & - & 0.04 & - & 0.01 & 0.31 & 0.11 \\
\hline POP2 & $\overline{\mathrm{x}}$ & 59.71 & 0.42 & 18.43 & 3.79 & 0.17 & 0.73 & 2.94 & 4.26 & 8.61 & 0.09 & 0.14 & 0.54 & 0.15 & 96.28 & 12.87 & 2.02 \\
\hline$n=26$ & SD & 1.10 & 0.07 & 0.11 & 0.62 & 0.05 & 0.25 & 0.57 & 0.17 & 0.34 & 0.05 & 0.07 & 0.06 & 0.06 & 1.34 & 0.48 & 0.07 \\
\hline RF93-77 $797 \mathrm{~cm}$ & $\overline{\mathrm{x}}$ & 59.41 & 0.41 & 19.17 & 3.69 & 0.10 & 0.77 & 2.84 & 4.36 & 8.75 & - & - & 0.50 & - & 97.24 & 13.11 & 2.01 \\
\hline$n=\mathrm{NA}$ & SD & 0.41 & 0.08 & 0.10 & 0.18 & 0.07 & 0.15 & 0.20 & 0.36 & 0.30 & - & - & 0.07 & - & 0.81 & 0.66 & - \\
\hline CM92-42 $710 \mathrm{~cm}$ & $\overline{\mathrm{x}}$ & 59.19 & 0.41 & 19.20 & 3.74 & 0.08 & 0.81 & 2.99 & 4.25 & 8.84 & - & - & 0.49 & - & 97.65 & 13.09 & 2.08 \\
\hline$n=\mathrm{NA}$ & SD & 0.39 & 0.07 & 0.17 & 0.22 & 0.05 & 0.11 & 0.22 & 0.30 & 0.23 & - & - & 0.07 & - & 1.46 & 0.53 & - \\
\hline OH-DP-0435 & $\overline{\mathrm{x}}$ & 61.06 & 0.45 & 18.53 & 3.09 & 0.30 & 0.36 & 1.78 & 6.12 & 6.98 & 0.05 & 0.37 & 0.86 & 0.05 & 96.82 & 13.10 & 1.16 \\
\hline$n=22$ & SD & 0.34 & 0.04 & 0.17 & 0.12 & 0.10 & 0.09 & 0.10 & 0.52 & 0.55 & 0.03 & 0.13 & 0.14 & 0.03 & 1.60 & 0.26 & 0.20 \\
\hline OT0702-9 & $\overline{\mathrm{x}}$ & 61.15 & 0.46 & 18.82 & 3.09 & 0.29 & 0.39 & 1.68 & 6.39 & 7.03 & - & - & 0.71 & - & 100.00 & 13.42 & 1.12 \\
\hline$n=15$ & SD & 0.30 & 0.06 & 0.12 & 0.14 & 0.09 & 0.10 & 0.08 & 0.62 & 0.50 & - & - & 0.14 & - & - & 0.20 & 0.20 \\
\hline JO575 & $\overline{\mathrm{x}}$ & 61.20 & 0.47 & 18.74 & 2.97 & 0.29 & 0.38 & 1.65 & 6.54 & 7.04 & - & - & 0.72 & - & 100.00 & 13.58 & 1.09 \\
\hline$n=20$ & SD & 0.43 & 0.08 & 0.17 & 0.15 & 0.08 & 0.08 & 0.11 & 0.61 & 0.45 & - & - & 0.12 & - & - & 0.32 & 0.17 \\
\hline $22 M-60 X-6$ & $\overline{\mathrm{x}}$ & 61.45 & 0.49 & 18.52 & 3.80 & 0.22 & 0.59 & 2.06 & 6.20 & 6.66 & - & - & - & - & 94.53 & 12.86 & 1.08 \\
\hline$n=\mathrm{NA}$ & SD & - & - & - & - & - & - & - & - & - & - & - & - & - & - & - & - \\
\hline TM27 & $\overline{\mathrm{x}}$ & 61.43 & 0.46 & 18.57 & 2.85 & 0.23 & 0.40 & 1.84 & 6.32 & 7.25 & 0.06 & - & 0.71 & - & 97.86 & 13.57 & 1.17 \\
\hline$n=22$ & SD & 0.40 & 0.02 & 0.09 & 0.13 & 0.06 & 0.08 & 0.11 & 0.66 & 0.50 & 0.04 & - & 0.14 & - & 1.40 & 0.24 & 0.19 \\
\hline POP4 & $\overline{\mathrm{x}}$ & 61.49 & 0.45 & 18.26 & 3.08 & 0.25 & 0.38 & 1.77 & 5.84 & 7.36 & 0.04 & 0.28 & 0.75 & 0.05 & 97.05 & 13.20 & 1.28 \\
\hline$n=31$ & SD & 0.42 & 0.03 & 0.11 & 0.11 & 0.07 & 0.06 & 0.08 & 0.52 & 0.43 & 0.02 & 0.10 & 0.14 & 0.03 & 1.31 & 0.28 & 0.17 \\
\hline S-10 & $\overline{\mathrm{x}}$ & 62.38 & 0.40 & 18.86 & 2.81 & 0.24 & 0.29 & 1.61 & 6.02 & 7.39 & - & - & - & - & - & 13.41 & 1.23 \\
\hline$n=\mathrm{NA}$ & SD & - & - & - & - & - & - & - & - & - & - & - & - & - & - & - & - \\
\hline I-9 & $\overline{\mathrm{x}}$ & 62.00 & 0.46 & 18.50 & 2.99 & 0.27 & 0.38 & 1.71 & 5.74 & 6.77 & 0.06 & 0.32 & 0.80 & - & 96.42 & 12.51 & 1.19 \\
\hline$n=15$ & SD & 0.39 & 0.05 & 0.24 & 0.09 & 0.05 & 0.04 & 0.07 & 0.31 & 0.30 & 0.04 & 0.13 & 0.10 & - & 0.83 & 0.22 & 0.11 \\
\hline KET8004-DED8708 C-31 & $\overline{\mathrm{x}}$ & 61.80 & 0.45 & 18.11 & 3.16 & 0.28 & 0.31 & 1.76 & 5.64 & 7.34 & 0.02 & 0.14 & 1.00 & - & - & 12.98 & 1.30 \\
\hline$n=\mathrm{NA}$ & SD & 0.50 & 0.09 & 0.19 & 0.17 & 0.11 & 0.16 & 0.21 & 0.54 & 0.60 & 0.03 & 0.08 & 0.25 & - & - & 1.14 & - \\
\hline KET8282 C-31 & $\overline{\mathrm{x}}$ & 61.74 & 0.49 & 19.19 & 3.10 & - & 0.43 & 1.72 & 6.03 & 7.31 & - & - & - & - & - & 13.34 & 1.21 \\
\hline$n=\mathrm{NA}$ & SD & 0.34 & 0.08 & 0.11 & 0.18 & - & 0.08 & 0.14 & 0.71 & 0.68 & - & - & - & - & - & 1.39 & - \\
\hline KET8222 C-31 & $\overline{\mathrm{X}}$ & 60.17 & 0.47 & 19.45 & 3.70 & - & 0.77 & 2.51 & 4.03 & 8.69 & - & - & - & - & - & 12.72 & 2.16 \\
\hline$n=\mathrm{NA}$ & SD & 0.12 & 0.04 & 0.04 & 0.20 & - & 0.06 & 0.25 & 0.55 & 0.62 & - & - & - & - & - & 1.17 & - \\
\hline PRAD2812 & $\overline{\mathrm{x}}$ & 62.00 & 0.46 & 18.84 & 2.93 & 0.24 & 0.39 & 1.75 & 6.10 & 7.29 & - & - & - & - & 96.21 & 13.39 & 1.22 \\
\hline$n=23$ & SD & 0.24 & 0.03 & 0.10 & 0.11 & 0.07 & 0.08 & 0.08 & 0.62 & 0.55 & - & - & - & - & 0.85 & 0.15 & 0.21 \\
\hline $\mathrm{C} 1202 \mathrm{t} 1$ & $\overline{\mathrm{x}}$ & 60.16 & 0.45 & 18.36 & 3.30 & 0.32 & 0.31 & 1.77 & 7.34 & 6.60 & 0.17 & 0.30 & 0.91 & - & 96.82 & 13.94 & 1.05 \\
\hline$n=20$ & SD & 0.28 & 0.12 & 0.25 & 0.22 & 0.11 & 0.08 & 0.11 & 0.21 & 0.21 & 0.12 & 0.18 & 0.06 & - & 1.81 & 0.26 & 0.03 \\
\hline
\end{tabular}


Table 1. Continued.

\begin{tabular}{|c|c|c|c|c|c|c|c|c|c|c|c|c|c|c|c|c|c|}
\hline & & $\mathrm{SiO}_{2}$ & $\mathrm{TiO}_{2}$ & $\mathrm{Al}_{2} \mathrm{O}_{3}$ & $\mathrm{FeO}_{\mathrm{TOT}}$ & $\mathrm{MnO}$ & $\mathrm{MgO}$ & $\mathrm{CaO}$ & $\mathrm{Na}_{2} \mathrm{O}$ & $\mathrm{K}_{2} \mathrm{O}$ & $\mathrm{P}_{2} \mathrm{O}_{5}$ & $\mathrm{~F}$ & $\mathrm{Cl}$ & $\mathrm{SO}_{3}$ & Total & Tot. alkali & Alk. ratio \\
\hline CIL2 & $\overline{\mathrm{x}}$ & 61.46 & 0.45 & 18.37 & 3.24 & 0.29 & 0.45 & 1.86 & 5.47 & 7.19 & 0.08 & 0.38 & 0.72 & 0.03 & 96.08 & 12.66 & 1.40 \\
\hline$n=74$ & $\mathrm{SD}$ & 0.67 & 0.05 & 0.34 & 0.25 & 0.09 & 0.19 & 0.28 & 0.97 & 0.97 & 0.06 & 0.20 & 0.23 & 0.04 & 1.51 & 0.42 & 0.47 \\
\hline SM1 & $\overline{\mathrm{x}}$ & 62.27 & 0.45 & 18.19 & 3.31 & 0.35 & 0.35 & 1.76 & 5.05 & 7.82 & - & - & 0.45 & - & 95.27 & 12.87 & 1.55 \\
\hline$n=15$ & $\mathrm{SD}$ & 0.33 & 0.04 & 0.25 & 0.16 & 0.01 & 0.14 & 0.06 & 0.18 & 0.35 & - & - & 0.07 & - & - & - & - \\
\hline SM2 & $\overline{\mathrm{X}}$ & 62.50 & 0.43 & 18.37 & 3.23 & 0.36 & 0.40 & 1.73 & 5.36 & 7.31 & - & - & 0.33 & - & 96.18 & 12.66 & 1.36 \\
\hline$n=11$ & $\mathrm{SD}$ & 0.90 & 0.02 & 0.18 & 0.24 & 0.14 & 0.20 & 0.22 & 0.23 & 0.50 & - & - & 0.11 & - & - & - & - \\
\hline SA & $\overline{\mathrm{X}}$ & 62.21 & 0.41 & 18.50 & 3.34 & 0.22 & 0.42 & 1.80 & 4.77 & 7.94 & - & - & 0.39 & - & 96.36 & 12.71 & 1.66 \\
\hline$n=16$ & $\mathrm{SD}$ & 0.24 & 0.07 & 0.17 & 0.09 & 0.10 & 0.07 & 0.09 & 0.21 & 0.16 & - & - & 0.10 & - & - & - & - \\
\hline OH-DP-0499 & $\overline{\mathrm{x}}$ & 65.32 & 0.69 & 15.32 & 6.02 & 0.28 & 0.33 & 1.32 & 5.68 & 4.92 & 0.11 & 0.08 & 0.15 & 0.06 & 97.13 & 10.61 & 0.87 \\
\hline$\overline{n=11 \text { (trachyte) }}$ & $\mathrm{SD}$ & 1.06 & 0.08 & 0.88 & 0.31 & 0.04 & 0.09 & 0.25 & 0.36 & 0.09 & 0.04 & 0.05 & 0.05 & 0.02 & 1.70 & 0.41 & 0.05 \\
\hline OH-DP-0499 & $\overline{\mathrm{x}}$ & 73.61 & 0.36 & 8.77 & 7.30 & 0.34 & 0.08 & 0.33 & 4.72 & 4.46 & 0.02 & 0.29 & 0.85 & 0.06 & 93.33 & 9.18 & 0.95 \\
\hline$\overline{n=32 \text { (pantellerite) }}$ & $\mathrm{SD}$ & 0.34 & 0.03 & 0.21 & 0.15 & 0.05 & 0.02 & 0.03 & 0.28 & 0.09 & 0.02 & 0.09 & 0.07 & 0.03 & 1.16 & 0.28 & 0.06 \\
\hline LC21 10.345 & $\overline{\mathrm{X}}$ & 73.91 & 0.39 & 8.65 & 7.06 & 0.30 & 0.03 & 0.29 & 5.01 & 4.36 & - & - & - & - & 100.00 & 9.37 & 1.06 \\
\hline$n=5$ & $\mathrm{SD}$ & 1.62 & 0.04 & 0.15 & 0.24 & 0.06 & 0.02 & 0.02 & 1.89 & 0.08 & - & - & - & - & - & 1.83 & 0.49 \\
\hline ODP3 & $\overline{\mathrm{x}}$ & 65.05 & 0.75 & 15.61 & 5.66 & 0.29 & 0.41 & 1.43 & 5.85 & 4.78 & 0.15 & 0.07 & 0.10 & - & 96.81 & 10.64 & 0.95 \\
\hline$n=14$ (trachyte) & $\mathrm{SD}$ & 0.33 & 0.04 & 0.20 & 0.16 & 0.04 & 0.03 & 0.07 & 0.31 & 0.15 & 0.02 & 0.04 & 0.02 & - & 1.39 & 0.30 & 0.06 \\
\hline ODP3 & $\overline{\mathrm{x}}$ & 73.58 & 0.39 & 9.40 & 6.63 & 0.32 & 0.10 & 0.31 & 4.83 & 4.43 & 0.02 & 0.24 & 0.68 & - & 94.78 & 9.25 & 1.40 \\
\hline$n=4$ (pantellerite) & SD & 0.98 & 0.02 & 1.21 & 0.83 & 0.02 & 0.05 & 0.01 & 0.61 & 0.11 & 0.01 & 0.08 & 0.17 & - & 2.21 & 0.64 & 0.10 \\
\hline TII5 & $\overline{\mathrm{x}}$ & 75.46 & 0.38 & 8.99 & 7.28 & 0.22 & 0.06 & 0.30 & 6.24 & 4.59 & - & - & - & - & 95.94 & 10.82 & 0.74 \\
\hline$n=15$ & $\mathrm{SD}$ & 0.57 & 0.04 & 0.14 & 0.28 & 0.09 & 0.02 & 0.03 & 0.30 & 0.26 & - & - & - & - & 0.69 & 0.31 & 0.85 \\
\hline ML5 & $\overline{\mathrm{x}}$ & 66.22 & 0.75 & 14.33 & 6.05 & 0.30 & 0.27 & 1.16 & 5.59 & 4.99 & - & - & - & - & 95.86 & 10.58 & 0.90 \\
\hline$n=6$ (trachyte) & $\mathrm{SD}$ & 0.93 & 0.06 & 0.98 & 0.43 & 0.02 & 0.09 & 0.24 & 0.25 & 0.12 & - & - & - & - & 1.33 & 0.15 & 0.06 \\
\hline ML5 & $\overline{\mathrm{X}}$ & 73.73 & 0.43 & 8.31 & 7.14 & 0.32 & 0.08 & 0.31 & 4.30 & 4.44 & & & & & 92.72 & 8.74 & 1.04 \\
\hline$n=50$ (pantellerite) & $\mathrm{SD}$ & 0.24 & 0.01 & 0.10 & 0.16 & 0.02 & 0.01 & 0.01 & 0.24 & 0.08 & & & & & 0.45 & 0.24 & 0.07 \\
\hline OT0702-10 & $\overline{\mathrm{x}}$ & 66.18 & 0.43 & 16.23 & 5.96 & 0.23 & 0.20 & 0.84 & 5.85 & 5.79 & - & 0.13 & - & - & 100.00 & 11.64 & 0.99 \\
\hline$n=3$ (trachyte) & $\mathrm{SD}$ & 0.73 & 0.13 & 2.54 & 0.08 & 0.16 & 0.17 & 0.10 & 0.64 & 1.14 & - & 0.09 & - & - & - & 1.44 & 0.18 \\
\hline ОТ0702-10 & $\overline{\mathrm{X}}$ & 72.52 & 0.41 & 9.34 & 6.70 & 0.31 & 0.12 & 0.34 & 5.06 & 4.50 & - & 0.71 & - & - & 100.00 & 9.55 & 0.91 \\
\hline$n=6($ pantellerite $)$ & $\mathrm{SD}$ & 0.72 & 0.08 & 1.12 & 0.36 & 0.05 & 0.06 & 0.06 & 0.64 & 0.28 & - & 0.07 & - & - & - & 0.56 & 0.17 \\
\hline JO941 & $\overline{\mathrm{X}}$ & 72.28 & 0.37 & 8.54 & 6.79 & 0.29 & 0.10 & 0.29 & 6.33 & 4.39 & - & 0.69 & - & - & 100.00 & 10.71 & 0.69 \\
\hline$n=13$ & $\mathrm{SD}$ & 0.26 & 0.11 & 0.09 & 0.10 & 0.09 & 0.21 & 0.05 & 0.18 & 0.09 & - & 0.05 & - & - & - & 0.14 & 0.48 \\
\hline KET8222 P-11 & $\overline{\mathrm{x}}$ & 64.67 & 0.83 & 15.50 & 5.88 & 0.28 & 1.45 & 6.41 & 4.98 & - & - & - & - & - & - & 11.39 & 0.78 \\
\hline$n=\mathrm{NA}$ (trachyte) & $\mathrm{SD}$ & 0.68 & 0.10 & 0.71 & 0.19 & 0.14 & 0.20 & 0.27 & 0.19 & - & - & - & - & - & - & 0.46 & - \\
\hline KET8222 P-11 & $\overline{\mathrm{x}}$ & 72.41 & 0.34 & 8.95 & 7.43 & 0.08 & 0.17 & 6.09 & 4.54 & - & - & - & - & - & - & 10.63 & 0.75 \\
\hline$n=\mathrm{NA}($ pantellerite $)$ & $\mathrm{SD}$ & 0.38 & 0.08 & 0.09 & 24.00 & 6.00 & 0.08 & 0.33 & 0.00 & - & - & - & - & - & - & 0.33 & - \\
\hline$O D P 2$ & $\overline{\mathrm{x}}$ & 63.92 & 1.00 & 16.22 & 5.35 & 0.25 & 0.99 & 2.50 & 5.55 & 3.89 & 0.35 & 0.09 & 0.24 & - & 97.22 & 9.44 & 0.82 \\
\hline$n=18$ & $\mathrm{SD}$ & 1.74 & 0.30 & 0.83 & 0.51 & 0.04 & 0.61 & 1.18 & 0.43 & 0.80 & 0.37 & 0.07 & 0.11 & - & 1.18 & 0.87 & 0.10 \\
\hline ODP4 & $\overline{\mathrm{x}}$ & 65.10 & 0.72 & 15.89 & 5.72 & 0.30 & 0.38 & 1.41 & 5.62 & 4.72 & 0.14 & 0.05 & 0.15 & - & 96.33 & 10.35 & 0.90 \\
\hline$n=33$ (trachyte) & $\mathrm{SD}$ & 0.61 & 0.04 & 0.47 & 0.14 & 0.04 & 0.08 & 0.12 & 0.38 & 0.12 & 0.04 & 0.05 & 0.23 & - & 1.55 & 0.39 & 0.06 \\
\hline$O D P 4$ & $\overline{\mathrm{x}}$ & 74.58 & 0.40 & 8.70 & 6.98 & 0.36 & 0.07 & 0.29 & 4.25 & 4.33 & 0.03 & 0.30 & 0.73 & - & 93.45 & 8.55 & 1.35 \\
\hline$n=33$ (pantellerite) & $\mathrm{SD}$ & 0.18 & 0.02 & 0.00 & 0.04 & 0.06 & 0.01 & 0.01 & 0.26 & 0.09 & 0.02 & 0.01 & 0.04 & - & 0.39 & 0.22 & 0.05 \\
\hline OH-DP-0617 & $\overline{\mathrm{x}}$ & 60.15 & 0.43 & 19.77 & 2.75 & 0.15 & 0.42 & 2.38 & 5.04 & 8.85 & 0.05 & 0.49 & 0.24 & 0.12 & 94.36 & 13.89 & 1.76 \\
\hline$\overline{n=13}$ & $\mathrm{SD}$ & 0.33 & 0.02 & 0.28 & 0.09 & 0.04 & 0.02 & 0.06 & 0.16 & 0.26 & 0.02 & 0.10 & 0.02 & 0.04 & 1.62 & 0.16 & 0.10 \\
\hline CF-V4 & $\overline{\mathrm{x}}$ & 58.90 & 0.48 & 20.46 & 3.20 & 0.18 & 0.62 & 2.71 & 5.00 & 8.37 & 0.08 & 0.47 & 0.25 & 0.11 & 95.85 & 13.37 & 1.68 \\
\hline$n=16$ & $\mathrm{SD}$ & 1.81 & 0.10 & 0.22 & 1.03 & 0.03 & 0.48 & 0.98 & 0.37 & 0.39 & 0.10 & 0.15 & 0.07 & 0.04 & 0.81 & 0.73 & 0.08 \\
\hline OT0701-6 & $\overline{\mathrm{x}}$ & 59.58 & 0.44 & 20.04 & 2.76 & 0.13 & 0.51 & 2.31 & 5.24 & 8.80 & - & - & 0.18 & - & 100.00 & 14.04 & 1.68 \\
\hline$n=15$ & $\mathrm{SD}$ & 0.22 & 0.07 & 0.12 & 0.14 & 0.09 & 0.08 & 0.09 & 0.15 & 0.16 & - & - & 0.04 & - & - & 0.10 & 0.08 \\
\hline Vico B & $\overline{\mathrm{x}}$ & 59.66 & 0.46 & 19.59 & 2.85 & 0.15 & 0.42 & 2.37 & 5.37 & 8.65 & 0.05 & 0.43 & 0.22 & 0.09 & 95.32 & 14.02 & 1.61 \\
\hline$n=13$ & $\mathrm{SD}$ & 0.26 & 0.07 & 0.10 & 0.05 & 0.04 & 0.03 & 0.05 & 0.09 & 0.16 & 0.04 & 0.11 & 0.02 & 0.03 & 1.31 & 0.14 & 0.05 \\
\hline OH-DP-0624 & $\overline{\mathrm{x}}$ & 51.40 & 1.02 & 18.17 & 8.00 & 0.19 & 3.09 & 8.36 & 3.20 & 5.88 & 0.68 & 0.19 & 0.34 & 0.37 & 97.77 & 9.08 & 1.84 \\
\hline$n=19$ & $\mathrm{SD}$ & 3.60 & 0.22 & 0.62 & 2.13 & 0.03 & 1.17 & 2.48 & 0.68 & 1.45 & 0.28 & 0.06 & 0.08 & 0.17 & 1.29 & 2.08 & 0.18 \\
\hline OT0701-7 & $\overline{\mathrm{x}}$ & 55.79 & 0.81 & 19.24 & 5.30 & 0.18 & 1.67 & 5.14 & 4.20 & 7.19 & 0.16 & - & 0.37 & - & 100.00 & 11.40 & 1.73 \\
\hline$n=22$ & $\mathrm{SD}$ & 3.92 & 0.28 & 0.63 & 2.49 & 0.08 & 1.25 & 2.67 & 0.71 & 1.58 & 0.16 & - & 0.07 & - & - & 2.27 & 0.13 \\
\hline PRAD3225 & $\overline{\mathrm{X}}$ & 57.82 & 0.68 & 19.12 & 4.15 & 0.19 & 1.05 & 4.05 & 4.88 & 8.08 & - & - & - & - & 97.21 & 12.95 & 1.65 \\
\hline$n=11$ & $\mathrm{SD}$ & 2.77 & 0.16 & 0.39 & 1.73 & 0.01 & 0.90 & 2.00 & 0.60 & 1.10 & - & - & - & - & 1.19 & 1.68 & 0.06 \\
\hline CF5 V5 & $\overline{\mathrm{X}}$ & 54.92 & 0.81 & 18.76 & 6.08 & 0.19 & 1.98 & 5.93 & 3.82 & 7.06 & 0.46 & 0.21 & 0.41 & 0.24 & 96.59 & 10.88 & 1.86 \\
\hline$n=14$ & $\mathrm{SD}$ & 3.56 & 0.22 & 0.58 & 2.12 & 0.05 & 1.06 & 2.09 & 0.64 & 1.12 & 0.30 & 0.08 & 0.08 & 0.08 & 1.39 & 1.71 & 0.13 \\
\hline C- 42 & $\overline{\mathrm{x}}$ & 49.74 & 1.49 & 18.06 & 8.63 & 0.27 & 3.26 & 8.25 & 3.37 & 5.97 & 0.79 & 0.17 & - & - & 99.56 & 9.34 & 1.77 \\
\hline$n=\mathrm{NA}$ & $\mathrm{SD}$ & 1.08 & 0.30 & 0.26 & 0.61 & 0.17 & 0.21 & 0.56 & 0.13 & 0.24 & 0.19 & 0.05 & - & - & - & 0.37 & - \\
\hline Pitigliano Tuff & $\overline{\mathrm{x}}$ & 58.89 & 0.60 & 19.65 & 3.42 & - & 0.15 & 0.78 & 3.69 & 4.16 & 8.59 & 0.08 & 0.21 & - & 98.41 & 12.75 & 2.09 \\
\hline$n=3$ & $\mathrm{SD}$ & 1.71 & 0.11 & 0.37 & 1.36 & - & 0.01 & 0.66 & 1.58 & 0.50 & 1.56 & 0.00 & 0.13 & - & 0.61 & 1.65 & 0.48 \\
\hline OH-DP-1817 & $\overline{\mathrm{x}}$ & 44.48 & 0.98 & 15.44 & 10.76 & 0.23 & 4.61 & 13.25 & 3.81 & 5.48 & 0.95 & 0.36 & 0.14 & 0.41 & 97.58 & 9.28 & 1.47 \\
\hline$n=10$ & $\mathrm{SD}$ & 1.60 & 0.12 & 0.97 & 1.02 & 0.06 & 0.44 & 1.40 & 0.59 & 0.47 & 0.13 & 0.11 & 0.02 & 0.18 & 0.85 & 0.77 & 0.24 \\
\hline AH-20-PRa & $\overline{\mathrm{x}}$ & 41.69 & 1.37 & 12.52 & 11.56 & 0.27 & 6.39 & 15.24 & 3.49 & 4.98 & 1.11 & 0.61 & - & 0.08 & 96.94 & 8.47 & 1.43 \\
\hline$n=6$ & $\mathrm{SD}$ & 0.16 & 0.04 & 0.05 & 0.17 & 0.03 & 0.30 & 0.13 & 0.08 & 0.36 & 0.12 & 0.11 & - & 0.02 & 0.44 & - & - \\
\hline Sulmona site 2 & $\overline{\mathrm{x}}$ & 41.92 & 1.19 & 14.23 & 12.50 & 0.32 & 5.20 & 14.20 & 4.00 & 5.43 & 1.02 & 0.49 & 0.16 & 0.16 & 96.95 & 9.43 & 1.37 \\
\hline$n=23$ & $\mathrm{SD}$ & 0.63 & 0.05 & 0.19 & 0.35 & 0.03 & 0.18 & 0.37 & 0.31 & 0.28 & 0.08 & 0.08 & 0.02 & 0.05 & 1.38 & 0.32 & 0.15 \\
\hline Sulmona site $2 \mathrm{a}$ & $\overline{\mathrm{X}}$ & 43.01 & 1.17 & 14.77 & 11.53 & 0.28 & 4.75 & 13.59 & 3.83 & 6.07 & 1.00 & 0.45 & 0.14 & 0.14 & 97.61 & 9.90 & 1.64 \\
\hline$n=30$ & $\mathrm{SD}$ & 1.40 & 0.15 & 0.94 & 1.16 & 0.06 & 1.01 & 1.57 & 0.41 & 1.46 & 0.13 & 0.12 & 0.02 & 0.07 & 1.12 & 1.21 & 0.60 \\
\hline Paganica site 5 & $\overline{\mathrm{x}}$ & 43.47 & 1.10 & 15.34 & 10.87 & 0.29 & 4.78 & 13.15 & 3.75 & 6.42 & 0.82 & 0.45 & 0.14 & 0.27 & 96.70 & 10.17 & 1.80 \\
\hline$n=18$ & $\mathrm{SD}$ & 1.86 & 0.16 & 1.76 & 1.20 & 0.07 & 1.19 & 2.17 & 0.98 & 1.32 & 0.17 & 0.16 & 0.05 & 0.12 & 1.22 & 1.70 & 0.54 \\
\hline
\end{tabular}


Table 1. Continued.

\begin{tabular}{|c|c|c|c|c|c|c|c|c|c|c|c|c|c|c|c|c|c|}
\hline & & $\mathrm{SiO}_{2}$ & $\mathrm{TiO}_{2}$ & $\mathrm{Al}_{2} \mathrm{O}_{3}$ & $\mathrm{FeO}_{\mathrm{TOT}}$ & $\mathrm{MnO}$ & $\mathrm{MgO}$ & $\mathrm{CaO}$ & $\mathrm{Na}_{2} \mathrm{O}$ & $\mathrm{K}_{2} \mathrm{O}$ & $\mathrm{P}_{2} \mathrm{O}_{5}$ & $\mathrm{~F}$ & $\mathrm{Cl}$ & $\mathrm{SO}_{3}$ & Total & Tot. alkali & Alk. ratio \\
\hline Raiano site 3 & $\overline{\mathrm{x}}$ & 43.68 & 1.06 & 14.49 & 10.62 & 0.27 & 5.24 & 13.69 & 3.53 & 6.53 & 0.88 & 0.44 & 0.13 & 0.21 & 97.79 & 10.06 & 1.85 \\
\hline$n=22$ & SD & 1.04 & 0.21 & 1.42 & 0.76 & 0.05 & 1.06 & 1.55 & 0.31 & 1.20 & 0.13 & 0.11 & 0.02 & 0.14 & 0.66 & 1.30 & 0.34 \\
\hline PR basal fallout & $\overline{\mathrm{x}}$ & 42.83 & 1.41 & 12.65 & 11.60 & 0.22 & 6.51 & 16.30 & 2.96 & 4.42 & 1.10 & 0.48 & - & 0.03 & 97.96 & 7.38 & 1.49 \\
\hline$n=10$ & SD & 0.17 & 0.11 & 0.11 & 0.09 & 0.04 & 0.10 & 0.29 & 0.06 & 0.11 & 0.06 & 0.14 & - & 0.03 & 0.86 & 0.14 & 0.04 \\
\hline CF7-V11 & $\overline{\mathrm{x}}$ & 43.49 & 1.03 & 14.81 & 10.83 & 0.26 & 4.58 & 13.15 & 4.23 & 6.67 & 0.94 & 0.38 & 0.14 & 0.30 & 99.04 & 99.04 & 10.90 \\
\hline$n=11$ & SD & 1.35 & 0.12 & 0.98 & 1.12 & 0.05 & 0.57 & 1.64 & 0.44 & 1.16 & 0.11 & 0.11 & 0.02 & 0.12 & 0.62 & 0.62 & 1.24 \\
\hline OH-DP-1955 & $\overline{\mathrm{x}}$ & 57.53 & 0.57 & 20.51 & 4.03 & 0.18 & 0.56 & 4.55 & 4.78 & 6.97 & 0.09 & 0.31 & 0.23 & 0.21 & 96.70 & 11.75 & 1.47 \\
\hline$n=18$ & SD & 1.49 & 0.10 & 0.47 & 0.34 & 0.04 & 0.07 & 0.52 & 0.72 & 1.55 & 0.02 & 0.11 & 0.05 & 0.05 & 1.83 & 1.90 & 0.33 \\
\hline SC5 & $\overline{\mathrm{x}}$ & 58.42 & 0.48 & 21.16 & 3.01 & 0.20 & 0.36 & 4.41 & 4.84 & 6.63 & 0.14 & 0.33 & 0.24 & 0.18 & 94.64 & 11.47 & 1.38 \\
\hline$n=17$ & SD & 1.37 & 0.09 & 0.98 & 0.74 & 0.08 & 0.16 & 0.52 & 0.52 & 0.69 & 0.10 & 0.18 & 0.10 & 0.14 & 1.55 & 0.91 & 0.20 \\
\hline Fall B & $\overline{\mathrm{x}}$ & 60.09 & 0.48 & 19.06 & 2.92 & 0.16 & 0.50 & 3.26 & 3.14 & 10.34 & 0.05 & - & - & - & 100.00 & 13.48 & 3.78 \\
\hline$n=6$ & SD & 0.25 & 0.01 & 0.09 & 0.08 & 0.02 & 0.04 & 0.17 & 0.15 & 0.12 & 0.01 & - & - & - & - & 0.25 & 0.16 \\
\hline OH-DP-2010 & $\overline{\mathrm{x}}$ & 54.03 & 0.76 & 18.14 & 6.33 & 0.15 & 1.77 & 6.23 & 3.08 & 8.66 & 0.35 & 0.32 & 0.08 & 0.49 & 97.59 & 11.73 & 2.87 \\
\hline$n=22$ & SD & 3.62 & 0.17 & 0.72 & 1.75 & 0.03 & 0.81 & 1.81 & 0.49 & 0.89 & 0.19 & 0.11 & 0.03 & 0.16 & 1.77 & 1.10 & 0.43 \\
\hline Fall A proximal & $\overline{\mathrm{x}}$ & 59.92 & 0.50 & 19.21 & 3.16 & 0.17 & 0.51 & 3.19 & 3.05 & 10.20 & 0.08 & - & - & - & 100.00 & 13.25 & 3.36 \\
\hline$n=11$ & SD & 0.29 & 0.02 & 0.14 & 0.12 & 0.11 & 0.05 & 0.25 & 0.17 & 0.17 & 0.03 & - & - & - & - & 0.26 & 0.20 \\
\hline $\mathrm{SC} 3$ & $\overline{\mathrm{x}}$ & 58.47 & 0.54 & 18.80 & 3.85 & 0.15 & 0.91 & 3.93 & 2.89 & 9.75 & 0.09 & 0.30 & 0.07 & 0.26 & 96.81 & 12.64 & 3.40 \\
\hline$n=20$ & SD & 1.31 & 0.04 & 0.18 & 0.72 & 0.03 & 0.33 & 0.78 & 0.25 & 0.61 & 0.04 & 0.12 & 0.02 & 0.13 & 1.33 & 0.73 & 0.29 \\
\hline A9 & $\overline{\mathrm{x}}$ & 58.87 & 0.55 & 19.13 & 3.60 & 0.12 & 0.59 & 3.57 & 3.65 & 9.81 & 0.11 & 0.28 & 0.14 & - & 97.10 & 13.46 & 2.73 \\
\hline$n=16$ & SD & 1.13 & 0.24 & 0.37 & 0.73 & 0.10 & 0.22 & 0.69 & 0.38 & 0.60 & 0.11 & 0.14 & 0.07 & - & 1.25 & 0.38 & 0.41 \\
\hline Sulmona 5-1c & $\overline{\mathrm{x}}$ & 60.13 & 0.44 & 19.05 & 3.01 & 0.14 & 0.55 & 3.16 & 3.09 & 10.33 & 0.06 & 0.00 & 0.11 & 0.38 & 95.15 & 13.42 & 3.36 \\
\hline$n=30$ & SD & 0.89 & 0.04 & 0.43 & 0.32 & 0.04 & 0.14 & 0.38 & 0.21 & 0.21 & 0.03 & 0.00 & 0.02 & 0.12 & 1.24 & 0.26 & 0.26 \\
\hline FIC-12.9 & $\overline{\mathrm{x}}$ & 55.52 & 0.63 & 19.41 & 4.75 & 0.19 & 1.28 & 5.34 & 4.38 & 8.27 & 0.23 & 0.43 & 0.11 & 0.31 & 96.51 & 12.65 & 1.95 \\
\hline$n=22$ & SD & 1.79 & 0.08 & 0.35 & 0.73 & 0.05 & 0.36 & 0.98 & 0.53 & 1.05 & 0.08 & 0.11 & 0.02 & 0.11 & 1.77 & 0.66 & 0.52 \\
\hline OH-DP-2017 & $\overline{\mathrm{x}}$ & 64.43 & 0.44 & 18.27 & 2.59 & 0.18 & 0.29 & 1.06 & 5.65 & 6.32 & 0.05 & 0.24 & 0.36 & 0.11 & 94.22 & 11.97 & 0.29 \\
\hline$n=21$ & SD & 0.20 & 0.08 & 0.12 & 0.08 & 0.03 & 0.02 & 0.05 & 0.26 & 0.14 & 0.03 & 0.07 & 0.02 & 0.03 & 0.60 & 1.12 & 0.06 \\
\hline A11 & $\overline{\mathrm{x}}$ & 63.68 & 0.46 & 18.28 & 2.73 & 0.18 & 0.42 & 1.44 & 5.17 & 7.13 & 0.15 & 0.11 & 0.24 & - & 96.52 & 12.30 & 1.41 \\
\hline$n=18$ & SD & 0.66 & 0.10 & 0.33 & 0.27 & 0.16 & 0.17 & 0.28 & 0.66 & 0.40 & 0.11 & 0.14 & 0.09 & - & 0.84 & 0.48 & 0.27 \\
\hline A12 & $\overline{\mathrm{x}}$ & 64.18 & 0.51 & 17.85 & 2.56 & 0.23 & 0.30 & 1.09 & 6.09 & 6.57 & 0.12 & 0.11 & 0.39 & - & 96.37 & 12.66 & 1.08 \\
\hline$n=17$ & SD & 0.53 & 0.12 & 0.23 & 0.23 & 0.16 & 0.09 & 0.20 & 0.33 & 0.20 & 0.12 & 0.13 & 0.08 & - & 0.92 & 0.31 & 0.08 \\
\hline $\mathrm{SC} 2$ & $\overline{\mathrm{x}}$ & 66.20 & 0.35 & 16.97 & 3.01 & 0.26 & 0.32 & 0.98 & 5.27 & 5.71 & 0.02 & 0.02 & 0.50 & 0.40 & 95.38 & 10.98 & 1.09 \\
\hline$n=16$ & SD & 0.58 & 0.03 & 0.17 & 0.21 & 0.03 & 0.13 & 0.26 & 0.27 & 0.12 & 0.02 & 0.01 & 0.04 & 0.10 & 1.21 & 0.32 & 0.05 \\
\hline OH-DP-2060 & $\overline{\mathrm{x}}$ & 44.94 & 0.78 & 18.67 & 8.44 & 0.29 & 1.61 & 10.91 & 5.39 & 7.74 & 0.28 & 0.91 & 0.19 & 0.95 & 96.34 & 13.13 & 1.46 \\
\hline$n=19$ & SD & 1.70 & 0.17 & 0.94 & 1.18 & 0.05 & 0.98 & 1.26 & 0.76 & 1.32 & 0.16 & 0.23 & 0.04 & 0.48 & 1.81 & 1.68 & 0.27 \\
\hline SUL 1-6 & $\overline{\mathrm{x}}$ & 44.73 & 0.82 & 19.48 & 8.17 & 0.36 & 1.45 & 10.90 & 5.59 & 8.31 & 0.18 & 0.85 & 0.18 & 0.75 & 96.73 & 13.90 & 1.53 \\
\hline$n=27$ & SD & 1.16 & 0.10 & 0.70 & 0.77 & 0.05 & 0.14 & 1.16 & 0.64 & 0.90 & 0.03 & 0.20 & 0.03 & 0.27 & 1.31 & 0.67 & 0.40 \\
\hline Oricola tuff & $\overline{\mathrm{x}}$ & 45.15 & 0.00 & 18.83 & 8.27 & 0.32 & 1.52 & 11.40 & 4.90 & 8.33 & 0.23 & 0.79 & 0.13 & 0.13 & 100.00 & 13.23 & 1.70 \\
\hline$n=8$ & SD & 0.66 & 0.00 & 0.31 & 0.45 & 0.03 & 0.11 & 0.62 & 0.25 & 0.67 & 0.04 & 0.09 & 0.02 & 0.05 & - & 0.76 & 0.15 \\
\hline TBA proximal & $\overline{\mathrm{x}}$ & 45.74 & 0.76 & 19.16 & 7.80 & 0.30 & 1.40 & 10.20 & 5.49 & 8.93 & 0.21 & 0.86 & - & 0.85 & 98.02 & 14.42 & 1.63 \\
\hline$n=7$ & SD & 0.79 & 0.11 & 0.14 & 0.19 & 0.03 & 0.06 & 0.46 & 0.27 & 0.29 & 0.03 & 0.14 & - & 0.10 & 0.70 & 0.27 & 0.12 \\
\hline \multicolumn{18}{|l|}{ Standard 1 - } \\
\hline USGS rhyolite & & & & & & & & & & & & & & & Total & & \\
\hline$n=2$ & $\overline{\mathrm{x}}$ & 75.84 & 0.23 & 11.16 & 2.23 & 0.16 & 0.05 & 0.09 & 4.57 & 4.51 & 0.03 & 0.25 & 0.18 & 0.03 & 99.32 & & \\
\hline Recommended & & 76.00 & 0.19 & 11.50 & 2.02 & 0.16 & 0.08 & 0.10 & 4.75 & 4.47 & - & - & - & 0.03 & 99.30 & & \\
\hline \multicolumn{18}{|l|}{ Standard 2 - } \\
\hline$n=2$ & $\overline{\mathrm{x}}$ & 50.79 & 0.87 & 8.36 & 0.09 & 6.53 & 16.27 & 15.95 & 1.29 & - & - & - & - & - & 100.14 & & \\
\hline Recommended & & 50.46 & 0.84 & 8.28 & 0.13 & 6.51 & 16.28 & 16.00 & 1.30 & - & - & - & - & - & 99.80 & & \\
\hline
\end{tabular}

OT0702-3 from Lake Ohrid (Vogel et al., 2010) and PT09152 from Lake Prespa (Damaschke et al., 2013). The geochemical composition (cf. Table 1, Fig. S1a in the Supplement) and the stratigraphic position of OH-DP-0027 suggest a correlation with the aforementioned cryptotephra horizons and their supposed origin, the Pomici di Mercato (PdM) eruption of the Somma-Vesuvius volcano. The PdM eruption is the only known eruption in the Late Pleistocene and early Holocene with such a composition in the central Mediterranean region (Mele et al., 2011; Santacroce et al., 2008).

The products of the PdM eruption are found as two distinct layers TM6a and TM6b in the Lago Grande di Montic- chio record (Wulf et al., 2004). The double layers were interpreted as being due to briefly interrupted eruptive phases of the eruption (Mele et al., 2011). OH-DP-0027 and the associated Ohrid cryptotephra horizons match better with TM6b (Table 1), which represents the main Plinian and the initial phase of the eruption (Wulf et al., 2004). A correlation with the PdM eruption is also established for tephra found in the Veliko Jezero on the island of Mljet (Croatia), where even more than two eruptive phases are indicated (Jahns and van den Bogaard, 1998). Tephra layers were correlated with the PdM eruption (cf. Fig. S1a) also in sediment cores from the Adriatic Sea, such as KET8218 (V1; Paterne et 
al., 1988), IN68-9, IN68-5, and RF95-11 (125, 259, $320 \mathrm{~cm}$, respectively; Calanchi and Dinelli, 2008), AD91-17 (190191, 195-196 cm; Marchini et al., 2014), or from MD90-918 (210-223 cm; Caron et al., 2012).

Santacroce et al. (2008) reviewed several existing ${ }^{14} \mathrm{C}$ ages on proximal deposits of the Mercato eruption and suggested a maximum age of $9010-8750 \mathrm{yr}$ cal BP. This age is somewhat younger than the varve age obtained from the Lago Grande di Monticchio record for TM6b $(9680 \pm 480$ years BP; Wulf et al., 2004). The most reliable age of $8630-8430 \mathrm{yr}$ cal BP comes probably from ${ }^{14} \mathrm{C}$ dating of charcoals collected at the basal part of the proximal fallout deposit (Zanchetta et al., 2011).

\subsection{OH-DP-0115-Y-3}

Tephra layer OH-DP-0115 $(11.492-11.507 \mathrm{mcd})$ is $1.5 \mathrm{~cm}$ thick, is brownish-greyish in colour, and has sharp upper and lower boundaries. OH-DP-0115 comprises both highly vesicular micro-pumices with elongated vesicles and bubble-wall and bubble-junction glass shards. The glass composition is mainly trachytic tending towards the phonolitic field in the TAS diagram (Fig. 3b, Table 1), which suggests a correlation with the prominent marine tephra layer Y-3 from the Campanian area (cf. Table 1, Fig. S1b; Keller et al., 1978; Albert et al., 2015). The slightly heterogeneous major element composition, with a low-silica $\left(<62 \mathrm{wt} . \% \mathrm{SiO}_{2}\right)$ and a high-silica (> 62 wt. $\% \mathrm{SiO}_{2}$ ) endmember is defined by Albert et al. (2015) as the diagnostic characteristic of the Y-3 tephra and perfectly matches the OH-DP-0115 tephra (cf. Fig. 3b, Table 1, Fig. S1b). The most probable source of the Y-3 tephra is the Campi Flegrei (CF) caldera (Di Vito et al., 2008; Zanchetta et al., 2008; Albert et al., 2015), but to date none of the suggested proximal counterparts of different eruptions could be unambiguously assigned as its origin (Albert et al., 2015). Furthermore, the previously most accepted correlation of the Y-3 tephra with the intracaldera VRa and the mid-distal SMP1e/CE1 deposits (Sulpizio et al., 2003; Di Vito et al., 2008) is rejected because new geochemical data gained on the Y-3 type locality (marine cores M25/4-12, RC9 191) separate the Y-3 from other CF eruptions of the Tufi Biancastri/NYT series (Tomlinson et al., 2012; Albert et al., 2015).

The Y-3 tephra is known as a widespread stratigraphic marker found in different archives in the central and eastern Mediterranean region, such as the Lago Grande di Monticchio (TM15; Wulf et al., 2004; Tomlinson et al., 2012), the southern Adriatic Sea core MD90-917 (920-17; Zanchetta et al., 2008), Lake Ohrid (OT0520-2; OT0700-1; OT07024; JO 187; summarized in Sulpizio et al., 2010), Lake Prespa (PT0915-5; Damaschke et al., 2013), and the Tenaghi Philippon peat record in Greece (TP 9.70; Albert et al., 2015). These correlations are validated by the low- and high-silica endmember (Albert et al., 2015). The correlations of the marine tephra layers C-7 (Tyrrhenian and Adriatic Sea; Paterne et al., 1988) and A2-B2 (C106-C45; Munno and Petrosino, 2004) and the terrestrial S-19 layer (Munno and Petrosino, 2007) with the Y-3 tephra were not revaluated by Albert et al. (2015) due to restricted data sets but seem to be likely.

Since no proximal equivalents could be unambiguously identified so far, the ages from these proximal correlations (e.g. 31-30 ka cal BP; Zanchetta et al., 2008) have to be rejected (Albert et al., 2015). However, a Bayesian age-depth model based on multiple radiocarbon ages above and below the TP 9.70 tephra of the Tenaghi Philippon record provides an age of 28.68-29.42 ka cal BP (Albert et al., 2015). This is probably the best age estimate, which is supported by radiocarbon ages from other distal archives, for example the ${ }^{14} \mathrm{C}$ age of 28.78-29.98 ka cal BP obtained at the top of the Ohrid tephra OT07042-4-Y-3 (Vogel et al., 2010). The Y-3 tephra represents an important marker in the Mediterranean area, linking marine and terrestrial archives close to the Marine Isotope Stage 3-2 transition and the North Atlantic Heinrich Stadial 3 (HS3).

\subsection{OH-DP-0169-Y-5 CI}

Tephra OH-DP-0169 (16.783-16.933 mcd) has a thickness of $15 \mathrm{~cm}$, which is the thickest tephra layer found within this part of the DEEP site sequence and can be visually separated into two units. The lower unit (OH-DP-0169a) has a sharp boundary at the bottom and is $2 \mathrm{~cm}$ thick and pale yellow in colour, whereas the upper unit (OH-DP-0169b) is $13 \mathrm{~cm}$ of pale brown, coarser material. The uppermost centimetres of the upper unit are mixed with lacustrine sediments and have an uneven top boundary, which is probably due to difficult penetration during coring. Both units comprise elongated vesicle-bearing micro-pumices and bubble-wall and bubblejunction shards with thick septa, and have the same trachytic to phonotrachytic glass composition (Fig. 3c, Table 1). The characteristic trachytic to phonotrachytic alkaline composition of OH-DP-0169 (cf. Figs. 3c and S1c) and its remarkable thickness allow an unambiguous correlation with the Campanian Ignimbrite (CI) eruption (Orsi et al., 1996; Civetta et al., 1997; Pappalardo et al., 2002) and the marine tephra layer Y5 (Keller et al., 1978; Thunell et al., 1979). The comparison of major element oxides such as $\mathrm{Al}_{2} \mathrm{O}_{3}, \mathrm{FeO}_{\mathrm{TOT}}$, and $\mathrm{CaO}$ strongly support the correlation with the Y-5-CI deposits and exclude other possible correlation with pre-CI deposits described in Tomlinson et al., 2012 (cf. Fig. S1c). Additionally, the CI-Y-5 was also recognized in previous studies at lakes Ohrid and Prespa (OT0520-3; OT0700-2; OT0701-1; OT0702-6; JO-244; PT0704-3 summarized in Sulpizio et al., 2010).

The proximal deposits of the CI are described to have a trimodal composition, which resulted from the different timing and dynamic of extraction and mingling of two layered, compositionally different magmas (Civetta et al., 1997; Pappalardo et al., 2002; Marianelli et al., 2006). According to Civetta et al. (1997), OH-DP-0169 belongs to the most 

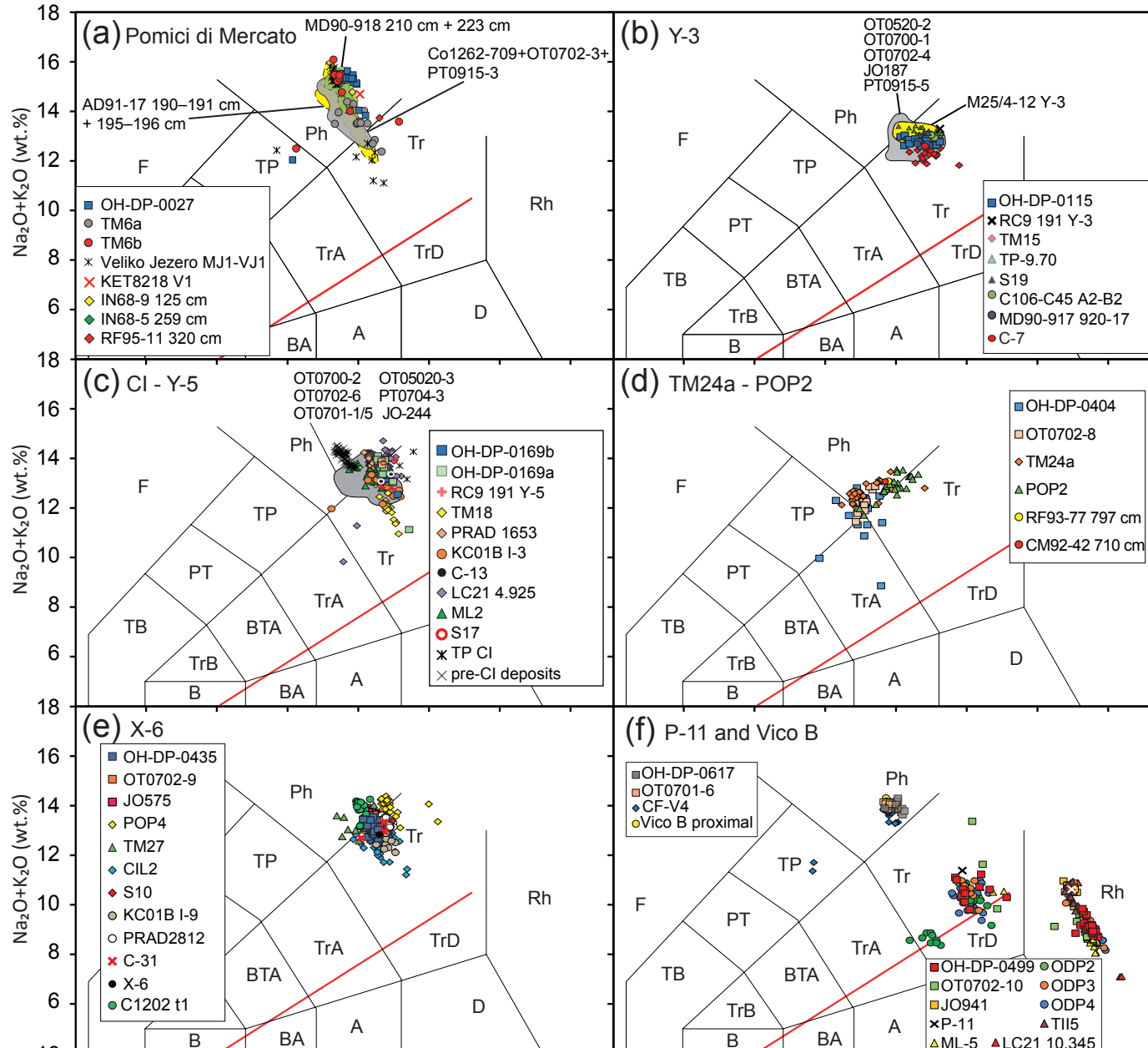

(f) P-11 and Vico $B$

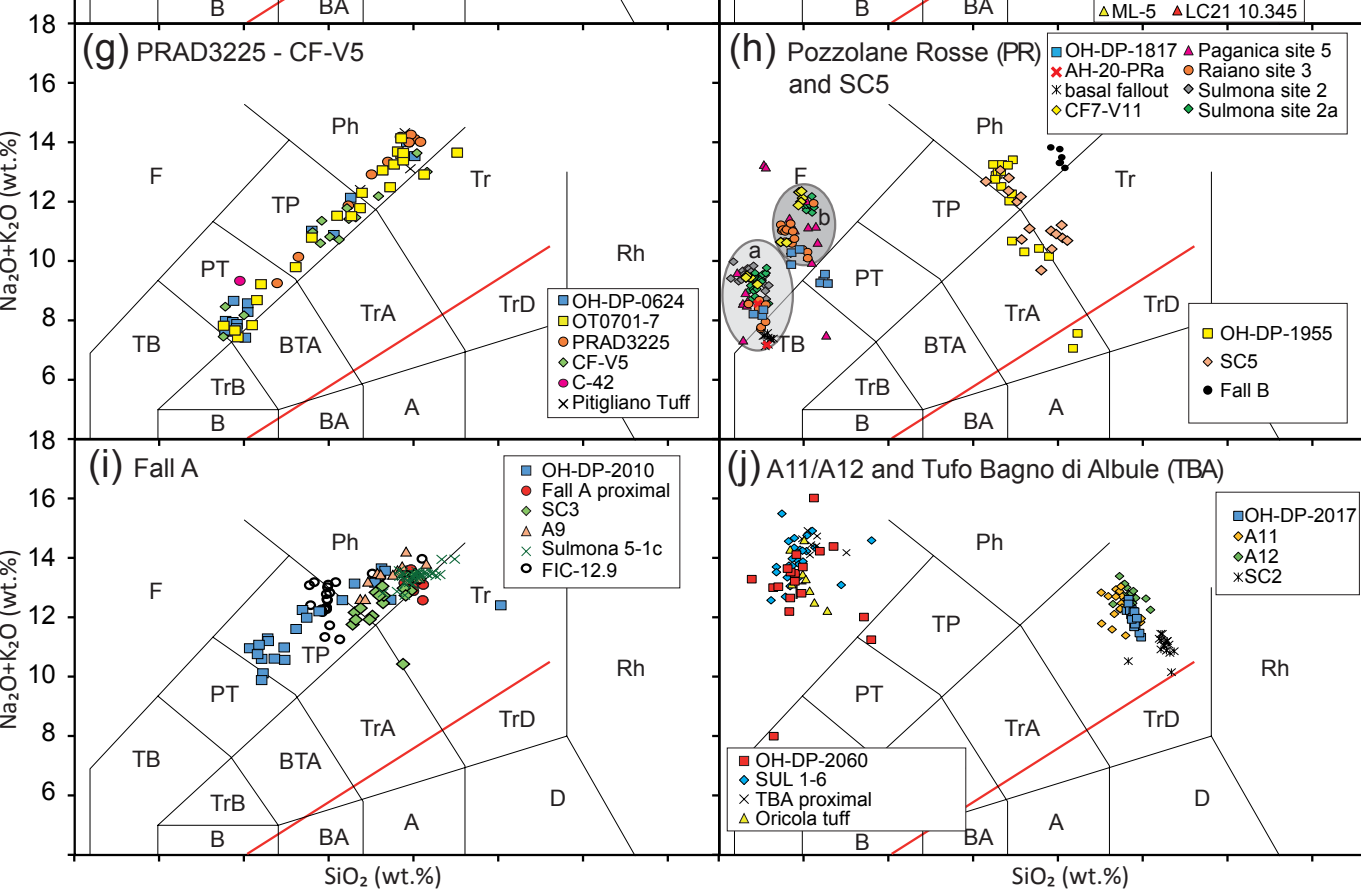

Figure 3. Total alkali-silica diagram after Le Bas et al. (1986) to classify and correlate the DEEP site tephra layers OH-DP-0027-OH-DP$2060(\mathbf{a}-\mathbf{j})$. The full data set of the DEEP site record is given in Table 1 and in the Supplement. Abbreviations and references used are found in Table A1 in the Apppendix. 
evolved composition $\left(\mathrm{K}_{2} \mathrm{O} / \mathrm{N}_{2} \mathrm{O}\right.$ 1.1-1.35) and only some shards can be assigned to the intermediate group $\left(\mathrm{K}_{2} \mathrm{O} / \mathrm{N}_{2} \mathrm{O}\right.$ 1.37-1.42; cf. Table $\mathrm{S} 1$ in the Supplement). The third, least evolved group $\left(\mathrm{K}_{2} \mathrm{O} / \mathrm{Na}_{2} \mathrm{O}>2\right)$ was not found in OH-DP0169 , most likely due to its relatively low abundance in distal settings and the limited number of analyses of OH-DP-0169. Indeed, the least evolved group is associated with the less energetic phase of the eruption of the late caldera collapse that generated more dense pyroclastic flows that did not reach a great distance from the vent (Civetta et al., 1997). Thus, they are likely less representative in very distal settings, also because the intermediate and most evolved group form more than $85 \%$ of the eruptive volume (Civetta et al., 1997).

As the two optical units of OH-DP-0169 (a and b) cannot be geochemically discriminated based on major elements, the initial Plinian phase (cf. Marianelli et al., 2006) may correspond with the lower, pale yellow and more fine-grained part (OH-DP-0169a), while the main ignimbrite phase probably corresponds with the upper, pale brown and coarser part (OH-DP-0169b).

The CI-Y-5 tephra layer, originating from the Campi Flegrei (e.g. Pappalardo et al., 2002), is the most widely dispersed tephra marker in the Mediterranean region. It was found, for example, in sediment cores from the Lago Grande di Monticchio (TM18; Wulf et al., 2004), the San Gregorio Magno Basin (S17; Munno and Petrosino, 2007), the Greek island of Lesvos (ML 2; Margari et al., 2007), and the Tenaghi Philippon peat record (TP CI; Lowe et al., 2012). Furthermore, it was identified in the Tyrrhenian Sea cores KET8022, KET8004, KET8003, KET8011 (C-13; Paterne et al., 1988; Ton-That et al., 2001), in the Adriatic Sea cores PRAD1-2 (PRAD1653; Bourne et al., 2010) and KET8218 (C-13; Paterne et al., 1988), in the Ionian Sea cores KC01B (I-3; Insinga et al., 2014), RC9 191, and V110 69 (Y-5; Keller et al., 1978), and in the Levantine and Aegean Sea cores (RC 9 183, -181, V10 58 (Y-5) - Keller et al., 1978; LC21 (4.925) - Lowe et al., 2012, Satow et al., 2015). To date, the most distal finding is in the Russian Plain, $2500 \mathrm{~km}$ away from its source (Pyle et al., 2006; Giaccio et al., 2008).

The ages for the CI cluster around $40 \mathrm{kaBP}$. The varve chronology of Lago Grande di Monticchio yielded an age of $36.77 \mathrm{ka} \mathrm{BP}$ (Wulf et al., 2006). ${ }^{40} \mathrm{Ar} /{ }^{39} \mathrm{Ar}$ dating on single sanidine crystals revealed ages of $37.1 \pm 0.4 \mathrm{ka}$ (Deino et al., 1994), $41.1 \pm 2.1 \mathrm{ka}$ (Ton-That et al. (2001), and $39.28 \pm 0.11 \mathrm{ka}$ (De Vivo et al., 2001), with the latter regarded as the best age, as it derives from proximal deposits.

\subsection{OH-DP-0404-TM24a POP2}

Tephra layer OH-DP-0404 (40.456-40.486 mcd) is a $3 \mathrm{~cm}$ thick dark reddish brown horizon with sharp stratigraphic contacts at the top and bottom. The layer comprises elongated vesicular micro-pumices and thick walled bubble-wall shards. Glass composition analyses reveal a mainly phonolitic composition with some shards scattering around the in- tersection of tephriphonolitic, trachyandesitic, and trachytic fields in the TAS diagram (Fig. 3d, Table 1). The geochemical signature is similar, even if somewhat more variable, to tephra OT-0702-8 (cf. Fig. 3d and S1d), found in Ohrid core Co1202 (Vogel et al., 2010), which is correlated with the Monticchio tephra TM24a (Wulf et al., 2004, 2012). The TM24a tephra was first correlated with the prominent marine X-5 layer (Keller et al., 1978), but this correlation was later revised and the older Monticchio tephra TM25 was correlated with the X-5 layer (Wulf et al., 2012). The proximal or the marine tephra counterparts of TM24a remain unknown so far. Based on major element compositions, OH-DP-0404 can be further correlated with the recently found POP2 tephra in the Popoli section from the Sulmona Basin (Regattieri et al., 2015), which is also correlated with the TM24a tephra (cf. Table 1, Fig. S1d). A correlation of OH-DP-0404 with marine tephra layers from core CM92-42 $(710 \mathrm{~cm})$ and core RF93-77 (797 cm), which were also correlated with TM24 (Calanchi and Dinelli, 2008), is likely; however, there is no differentiation between the bifurcation of tephra TM24 $(a+b)$ in these marine tephra layers. According to the Monticchio varve record, TM24a has an age of $101.8 \pm 5 \mathrm{kaBP}$ (Wulf et al., 2012), which matches the age of $102.0 \pm 2.4 \mathrm{ka}$ obtained from the age model of the Sulmona Basin section (Regattieri et al., 2015).

\subsection{OH-DP-0435-X-6}

Tephra OH-DP-0435 (43.498-43.513 mcd) is a $1.5 \mathrm{~cm}$ thick, greyish brown layer with sharp top and bottom contacts. The horizon comprises highly vesicular micro-pumices and bubble-wall shards with thick septa having a low alkali ratio (LAR) trachytic glass composition with only few shards plotting in the phonolitic field. This geochemical composition and the position below OH-DP-0404-TM24a-POP2 suggest a correlation of OH-DP-0435 with the marine tephra layer X-6 (core 22M-60; Keller et al., 1978). The correlation is supported by the geochemical similarity of OH-DP-0435 with the Ohrid tephra layers OT0702-9 (Vogel et al., 2010) and JO-575 (Caron et al., 2010), which were also correlated with the X-6 layer (cf. Figs. 3e and S1e). Equivalents of the X-6 tephra are also found in marine cores KET8004 and KET8022 of the Tyrrhenian Sea (C-31; Paterne et al., 2008), the Adriatic Sea cores PRAD1-2 (PRAD2812; Bourne et al., 2015), and the Ionian Sea cores KC01B (I-9; Insinga et al., 2014) and KET82-22 (C-31; Paterne et al., 2008). Furthermore, the X-6 is known from the terrestrial records of the Lago Grande di Monticchio (TM27; Wulf et al., 2012), the San Gregorio Magno Basin (S10; Munno and Petrosino, 2007), the Cilento coastline (SM1-SM2-SA - Marciano et al., 2008; CIL2 - Giaccio et al., 2012), and the Popoli section of the Sulmona Basin (POP4; Regattieri et al., 2015). The origin of the X-6 is assigned to an unknown eruption of the Campanian area (Keller et al., 1978; Paterne et al., 2008; Wulf et al., 2012). 
Based on ${ }^{40} \mathrm{Ar} /{ }^{39} \mathrm{Ar}$ dating, the age of tephra layer X-6 is $107 \pm 2 \mathrm{ka}$ (Kraml, 1997), which fits in with the varvebased age of ca. 108.3 $\pm 5.4 \mathrm{ka} \mathrm{BP}$ of the TM27 layer in Lago Grande di Monticchio (Wulf et al., 2012) and the interpolated age of $107 \mathrm{ka}$ of the C-31 tephra layer (Paterne et al., 2008). More recently, an ${ }^{40} \mathrm{Ar} /{ }^{39} \mathrm{Ar}$ age of $108.9 \pm 1.8 \mathrm{ka}$ was obtained on X-6 equivalent deposits of the Cilento offshore area (t1 tephra; Iorio et al., 2014), which matches the interpolated age of the Popoli section well $(109.0 \pm 1.5 \mathrm{ka}$; Regattieri et al., 2015).

\subsection{OH-DP-0499-P-11}

The OH-DP-0499 (49.937-49.947 mcd) tephra layer is $1 \mathrm{~cm}$ thick, olive brown, and characterized by a sharp bottom and a diffuse top boundary. It comprises elongated vesicular micropumices and larger platy bubble-wall shards. OH-DP-0499 has a distinct bimodal glass chemical composition, with a clearly separated trachytic and a rhyolitic group (Fig. 3f, Table 1). The shards can be classified as comendites and pantellerites (cf. $\mathrm{Al}_{2} \mathrm{O}_{3}-\mathrm{FeO}_{\text {Tот }}$ diagram; Fig.4), which unambiguously assign them to an origin in Pantelleria, the only source of these type of magmas in the central Mediterranean region (Peccerillo, 2005). The 2-fold composition is explained by a chemically zoned magma chamber, in which peralkaline rocks originating from mantle-derived parental magma and trachytic magma are differentiated in a low-pressure magma chamber by crystal-liquid fractionation (Civetta et al., 1984).

The stratigraphic position of OH-DP-0499, below the X6 tephra (OH-DP-0435), infers a definite correlation with the P-11 tephra (cf. Fig. 3f) found in the Ionian Sea core KET8222 (Paterne et al., 2008), since other widespread eruptions of Pantelleria are much younger (ca. $77 \mathrm{ka}$; P-10; Paterne et al., 2008; $45.7 \mathrm{ka}$, Green Tuff, Scaillet et al., 2013). Furthermore, the geochemical fingerprint of OH-DP-0499 is identical to those of the two cryptotephra layers OT0702-10 (Vogel et al., 2010) and JO-941 (Caron et al., 2010), which were found previously in Lake Ohrid sediments and correlated with P-11 (cf. Fig. S1f). A more proximal core from the Sicilian Channel (ODP 963A) comprises three pantelleritic layers (ODP2-4) in a similar stratigraphic position (Tamburrino et al., 2012). ODP2 shows a somewhat different geochemical composition (benmoritic part) compared to $\mathrm{OH}-$ DP-0499-P-11 (cf. Table 1, Fig. S1f). ODP3 and ODP4 indicate a very similar composition, but ODP3 is formed as a distinct horizon, whereas ODP4 is a cryptotephra (Tamburrino et al., 2012). Due to chronological concerns, Tamburrino et al. (2012) correlated ODP3 with P-11, which is supported by their climastratographic position at the transition from MIS 6 to 5 (cf. Zanchetta et al., 2015). As only one pantelleritic layer is found in distal archives, ODP2 was precluded due to chemical considerations, and ODP4 is a cryptotephra, the most likely correlation is of ODP 3 with the P-11 layer, being therefore the most widespread tephra.

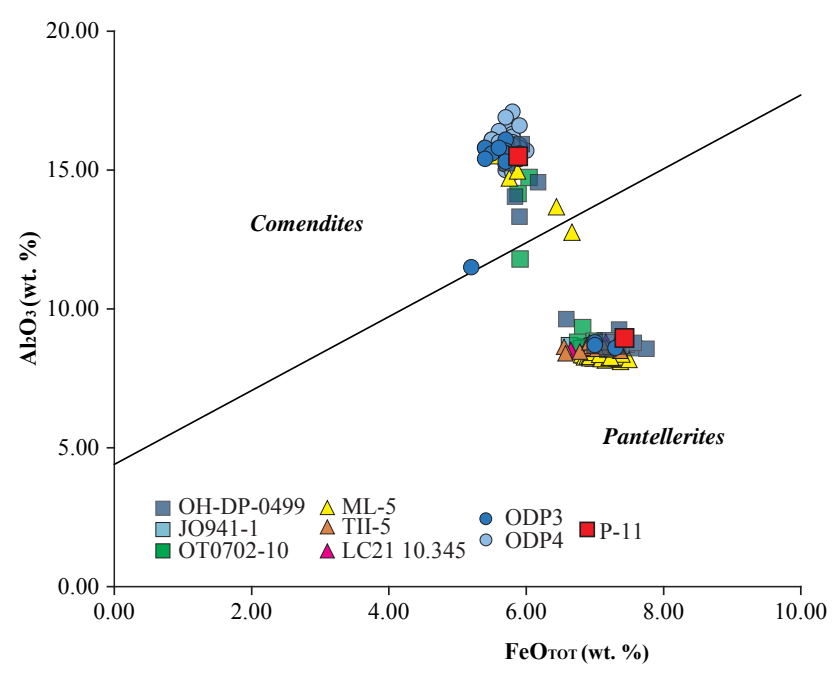

Figure 4. $\mathrm{Al}_{2} \mathrm{O}_{3}-\mathrm{FeO}_{\mathrm{TOT}}$ diagram for classification of comendites and pantellerites according to MacDonald (1974). Tephra OHDP-0499 and most of the other P-11 equivalents show the typical bimodal chemical composition of P-11, except for the very distal equivalents LC21 10.345 and THP-TII5, which only have a pantelleritic part (see text for data references).

OH-DP-0499 can be further correlated with the ML5 tephra found on Lesvos (Margari et al., 2007), as already suggested by Vogel et al. (2010) for the OT0702-10 tephra. The ML5 tephra was previously correlated with the younger Green Tuff-Y-6, but the geochemical data support a correlation with the older P-11 tephra. In more recent studies, Karkanas et al. (2015) correlated a pantelleritic cryptotephra (THP-TII5) found in the Theopetra cave in central Greece with P-11 and its equivalents. Satow et al. (2015) found a cryptotephra (LC21 10.345) in the Aegean Sea core LC21, which he ascribed to one of the ODP2-4 tephra layers. The position of LC21 10.345 in the G. ruber $\delta^{18} \mathrm{O}$ record of LC21 implies a correlation with ODP3 and P-11 based on the position in the respective isotope records. Nevertheless, it has to be stated that only the rhyolitic endmember of P-11 was found in the records from LC21 and Theopetra. The typical comenditic trachytic part is not found in these archives, which is probably due to a different dispersal of the 2-fold zonation of the magma chamber (rhyolitictrachytic part) tapped at different phases of the eruption. This is also indicated by an internal zonation of the OPD3 layer. Whereas the bottom of ODP3 has a pantelleritic composition, its top shows comenditic trachytic compositions (Tamburrino et al., 2012). This suggests that the rhyolitic pantelleritic part was erupted first and dispersed over a larger area, while the comenditic trachytic part is distributed only in a smaller, northern sector. Changes in the plume direction and dispersal during an eruption are likely due to changes in the aerodynamic characteristics of the erupted material or in the 
high- and low-atmosphere dynamics (Sulpizio et al., 2008, 2013).

Proximal counterparts of $\mathrm{P}-11$ are the ignimbrite deposits of the P unit on Pantelleria (Mahood and Hildreth, 1986; Paterne et al., 2008; Tamburrino et al., 2012). These proximal $\mathrm{P}$ unit deposits provide inhomogeneous ${ }^{40} \mathrm{Ar} /{ }^{39} \mathrm{Ar}$ ages between $123 \pm 1.6 \mathrm{ka}$ and $135 \pm 1.2 \mathrm{ka}$, with large internal age variations suggesting xenocrystic contamination (Rotolo et al., 2013). The mean age of the $P$ unit $(129 \pm 5.9 \mathrm{ka})$ is in broad accordance with the non-radiometric ages from the distal deposits. The age-depth model of core KET82-22, based on orbital tuning of the oxygen isotope and sapropel stratigraphy, provided an age of $130.6 \pm 5 \mathrm{ka}$ for the P-11 tephra (Paterne et al., 2008). This age matches the age of $128.1 \mathrm{ka}$ of ODP3 well, which is inferred from the correlation of benthic and planktonic foraminifera $\delta^{18} \mathrm{O}$ curves of core ODP-963A with the SPECMAP stack curve (Incarbona et al., 2008; Tamburrino et al., 2012). The correlation of LC21 10.345 with P-11 provides an age of $133.5 \pm 2 \mathrm{ka}$, based on the correlation of the surface-water foraminiferal $\delta^{18} \mathrm{O}$ record of LC21 with the high-resolution U-Th-dated Soreq Cave $\delta^{18} \mathrm{O}$ record (Grant et al., 2012, Satow et al., 2015).

\subsection{OH-DP-0617-Vico Ignimbrite B}

The tephra layer OH-DP-0617 (61.701-61.726 mcd) is a yellowish brown, $2.5 \mathrm{~cm}$ thick deposit with sharp bottom and top contacts. The very fine-grained glass shards have various forms, being highly vesicular with circular and oval bubbles. Micro-pumices with elongated vesicles are rare. The characteristic homogenous phonolitic composition of OH-DP0617 (Fig. 3f, Table 1) is very similar to the composition of tephra OT0701-6 (cf. Fig. 5a), which was found in the Ohrid core Co1201 (Sulpizio et al., 2010). OT0701-6 was previously correlated with the marine tephra C-20 and the proximal deposits of the SA3-b eruption from the Campanian area (Sulpizio et al., 2010). However, the stratigraphic position of OH-DP-0617 below the X-6-C-31-OH-DP-0435 and P11-OH-DP-0499 tephra suggests that and OH-DP-0617 and OT0706-1 instead correlate with the CF-V4 tephra from the Campo Felice Basin (Giraudi et al., 2011). The CF-V4 tephra is correlated with the "Ignimbrite B" of the Vico volcano (Vico B, Ronciglione Formation; Bear et al., 2009), which also has a large geochemical similarity with the Ohrid tephra layers OH-DP-0617-OT0701-6 (cf. Table 1, Fig. 5a). So far, the equivalents of this Vico eruption were not found in other archives. Laurenzi and Villa (1987) ${ }^{40} \mathrm{Ar} /{ }^{39} \mathrm{Ar}$ dated the Ignimbrite $\mathrm{B}$ in a proximal setting to $157 \pm 3 \mathrm{ka}$.

\subsection{OH-DP-0624-CF-V5-PRAD3225}

Tephra OH-DP-0624 $(62.367-62.413 \mathrm{mcd})$ is $4.6 \mathrm{~cm}$ thick, is of olive brown colour, and has a sharp bottom and more diffuse top transition. It comprises micro-pumices with elongated vesicles, cuspate glass shards, and non-vesicular, blocky, porphyritic particles, bearing prismatic microlites and phenocrysts. In the uppermost diffuse part, volcanic fragments are mixed with authigenic siderite crystals. The glass composition spans the phonotephritic and the phonolitic field of the TAS diagram (Fig. 3g, Table 1). This characteristic is also observed for tephra OT0701-7 in core Co1201 from Lake Ohrid (Sulpizio et al., 2010). OT0701-7 was first subdivided into three different chemical groups (a-b-c), of which OT0701-7b was tentatively correlated with the tephra layer OT0702-8-TM24a-X-5 (Sulpizio et al., 2010). However, this correlation is not supported by the stratigraphic position of OT0701-7 below OT0701-6, as the latter correlates well with OH-DP-0617. Furthermore, OT0702-8-TM24a is correlated with OH-DP-0404, which is embedded by sediments of interglacial MIS 5, whereas glacial sediments encompass OT0701-7 (Sulpizio et al., 2010). The glass composition of OT0701-7, showing a linear geochemical trend rather than two different geochemical populations, also makes a correlation with OH-DP-0624 more likely (Fig. 5b).

The peculiar geochemical trend of OH-DP-0624 (cf. Figs. 3g and 5b) matches the marine tephra PRAD3225 from the Adriatic Sea (Bourne et al., 2015), which is tentatively correlated with the tephra 322 from core RF95-7 and with the TM38 tephra from the Lago Grande di Monticchio record (Wulf et al., 2012). Tephra 322 was assigned to the Vico D eruption (Calanchi and Dinelli, 2008), which is dated to ca. $138 \mathrm{ka}$ (Laurenzi and Villa, 1987). TM38 has an age of $125.6 \pm 6.3 \mathrm{ka}$ according to the varve chronology of the Lago Grande di Monticchio record (Wulf et al., 2012). However, the ages of TM38 and 322-Vico D are significantly too young for the stratigraphic position of OH-DP-0624 below the OH-DP-0617-Vico B and dated to ca. $157 \pm 3 \mathrm{ka}$. Another marine counterpart of OH-DP-0624 could be tephra C42 with its phonotephritic composition (core DED8708; Paterne et al., 2008), but the published average values do not allow a robust correlation.

The most reliable equivalent of OH-DP-6024-OT0701-7PRAD3225 is probably found in the Campo Felice Basin in the Apennine chain. The geochemical composition and the stratigraphic position of the CF-V5 tephra, which was found in this basin directly below the CF-V4 tephra (Giraudi and Giaccio, 2015) correlates well with the composition and stratigraphic position of the OH-DP-6024 directly below the OH-DP-0617 tephra. Giraudi and Giaccio (2015) tentatively suggest that the CF-V5 tephra is an equivalent of the Pitigliano Tuff from the Latera caldera in the Vulsini volcanic complex (Turbeville, 1992a). Although the geochemical data set is limited, a similar characteristic trend is observed in CFV5 and OH-DP-0624-OT071-7-PRAD3225 (cf. Fig. 5b). ${ }^{40} \mathrm{Ar} /{ }^{39} \mathrm{Ar}$ dating on proximal deposits of the Pitigliano Tuff provided ages of $158 \pm 11 \mathrm{ka}$ and $155 \pm 11 \mathrm{ka}$ (Turbeville, 1992a, b) and the overlying Vico Ignimbrite B tephra limits the minimum age to $157 \pm 3 \mathrm{ka}$. 
(a) OH-DP-0617 - Vico B
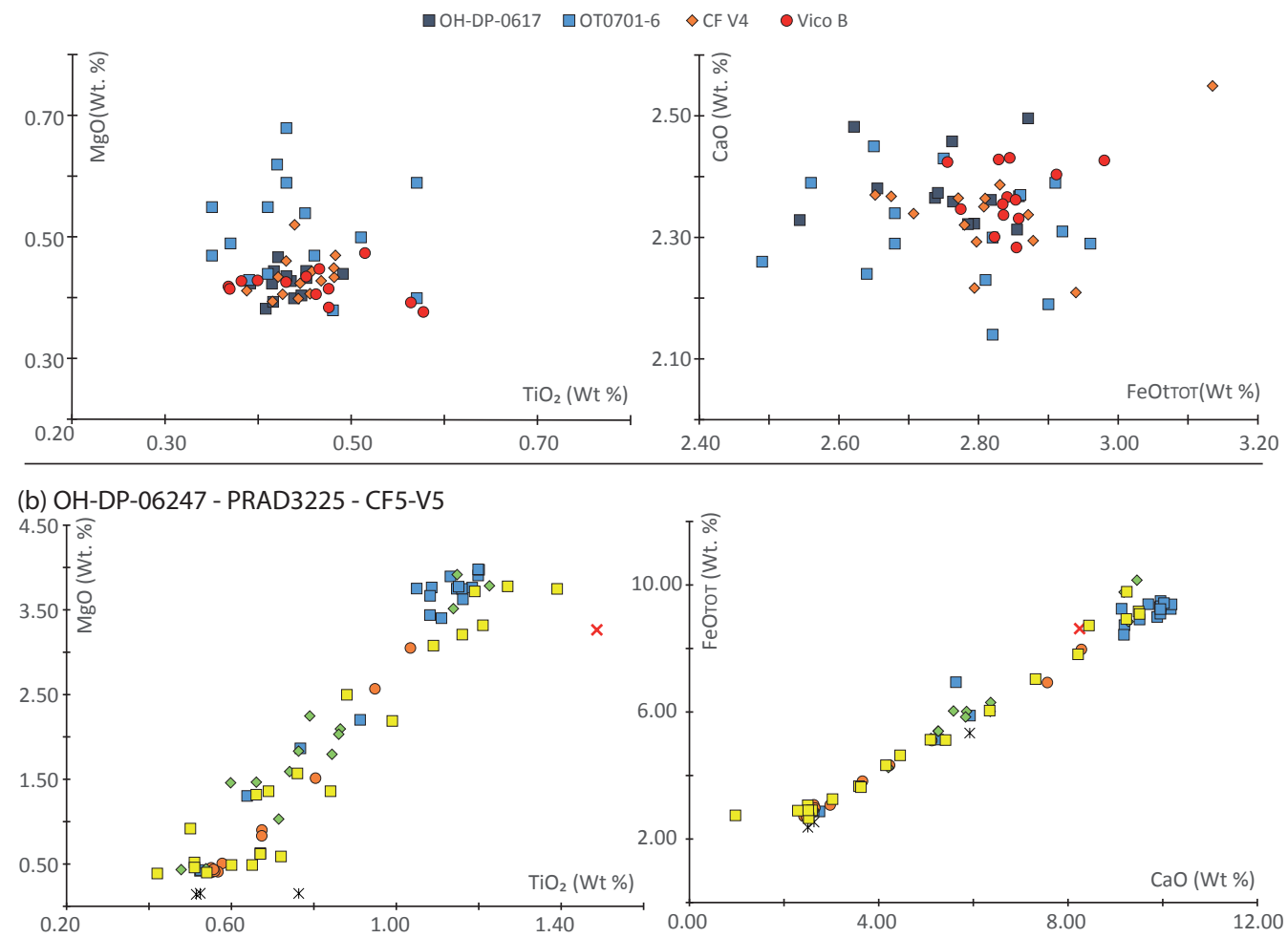

$\square$ OH-DP-0624 $*$ Pittigliano Tuff OPRAD3225 $\diamond$ CF5 V5 $\times$ C-42 $\square$ 口T0701-7
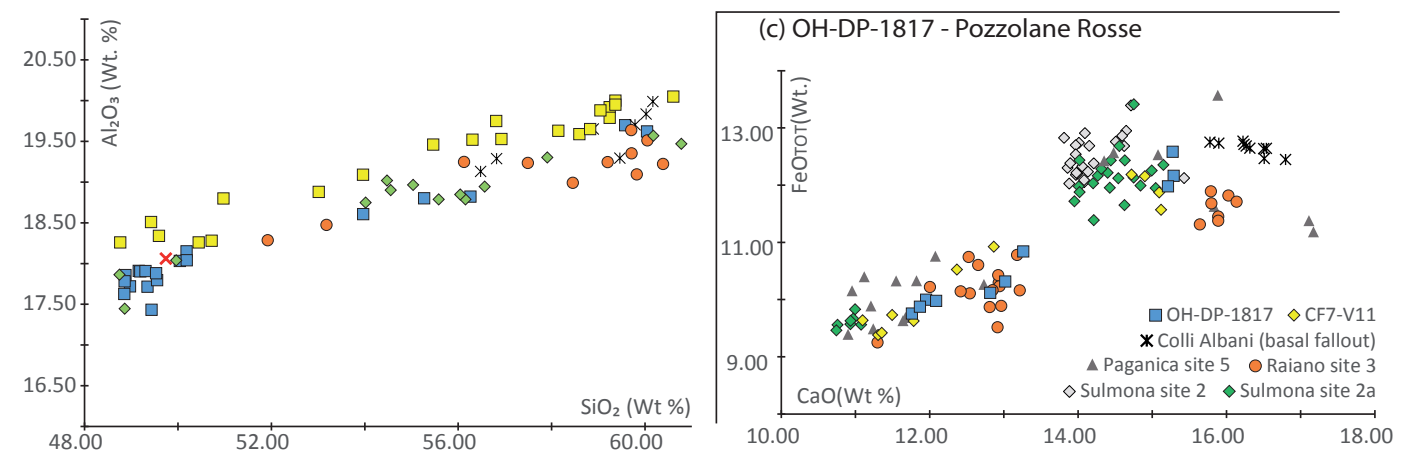

Figure 5. Harker diagrams of tephra layers OH-DP-0617 to OH-DP-1817 and their discussed equivalents. For references of correlated tephra layers, see text; additional Harker diagrams for OH-DP-1817 can be found in Fig. S1g.

\subsection{OH-DP-1817-Pozzolane Rosse}

Tephra layer OH-DP-1817 (181.744-181.769 mcd) is a dark brownish layer with a thickness of $2.5 \mathrm{~cm}$ and sharp bottom and top contacts. The layer contains mostly non-vesicular, blocky porphyritic shards with a high content of microlites and phenocrysts, mostly leucite. Vesicular cuspate shards are rare and are also porphyritic. OH-DP-1817 has a poorly evolved K-foiditic composition (Fig. 3h, Table 1), which is only known from the Italian volcanoes of the Colli Albani volcanic district and more sporadically in the Monte Vulture volcano (Peccerillo, 2005). However, as the activity of the Monte Vulture volcano clustered between $\sim 740$ and $610 \mathrm{ka}$ and around $140 \mathrm{ka}$ (Villa and Buettner, 2009), the most relevant source of foiditic tephra is the Colli Albani caldera, the Middle Pleistocene explosive activity of which was characterized by very high-energetic events (e.g. Marra et al., 2009). The most widespread Colli Albani tephra is from the Pozzolane Rosse eruption (Giaccio et al., 2013a) of the Tuscalano-Artemisio phase ( 560-360 ka; Marra et al., 2011).

A direct chemical correlation of OH-DP-1817 with proximal Pozzolane Rosse pyroclasts is difficult because of the lack of a comprehensive geochemical data set. Marra et al. (2009) describe the proximal type localities (within the Colli Albani volcanic district) of the Pozzolane Rosse py- 
roclastic products, but only the mean value of one geochemical analysis is published (AH-20-PRa). Another, more comprehensive data set for the composition of the proximal Pozzolane Rosse pyroclastics was provided by Freda et al. (2010), who, however, only reported the composition of the basal sub-Plinian fallout. Therefore, the correlation OH-DP-1817-Pozzolane Rosse (cf. Figs. 3h, 5c and S1g) is mainly based on the major element data of the ${ }^{40} \mathrm{Ar} /{ }^{39} \mathrm{Ar}$ dated distal equivalents of the Pozzolane Rosse units, which occur diffusely in the fluvial-lacustrine successions of the Apennine intermountain basins of Paganica-San DemetrioCastelnuovo and Sulmona, east of the Colli Albani (Galli et al., 2010; Giaccio et al., 2013a). Giaccio et al. (2013a) separate the Pozzolane Rosse distal equivalents into two sublayers, which differ chemically and morphologically. OH-DP1817 matches both compositions of the less evolved composition (group a) of the lower layer and the more evolved composition (group b) of the upper sublayer (cf. Fig. 3h). Besides the geochemical correlation, microtextural features, such as the scoria character (dense, few vesicles) of the shards and the huge number of juvenile crystals, suggest that the components of the lower sublayer prevail in OH-DP-1817. Giaccio et al. (2013a) correlate the lower sublayer (group a) to the proximal Pozzolane Rosse basal fallout and the upper sublayer to the phoenix cloud of the main pyroclastic flowforming phase. According to Freda et al. (2011), the Pozzolane Rosse eruption sequence encompasses from bottom to top the Vallerano lava flow, the main Pozzolane Rosse pyroclastic units (basal fallout and main pyroclastic flow unit), and the scoria lapilli fallout deposits. Recently, the products of the Pozzolane Rosse eruption were also found in the Campo Felice Basin (layer CF-V11), where it marks the local glacier advancement of the MIS 12 glacial (Giraudi and Giaccio, 2015). Pronounced glacial conditions are also recorded in the lacustrine sediments across the Pozzolane Rosse of the Sulmona Basin (Regattieri et al., 2016).

The age of the Pozzolane Rosse eruption is well constrained by several ${ }^{40} \mathrm{Ar} /{ }^{39} \mathrm{Ar}$ ages. The pyroclastic products are dated to $457 \pm 4 \mathrm{ka}$ at a proximal site (Karner et al., 2001), which is confirmed by the distal equivalent found in the Sulmona Basin (457 $\pm 2 \mathrm{ka}$, Giaccio et al., 2013a). These ages are further supported by ${ }^{40} \mathrm{Ar} /{ }^{39} \mathrm{Ar}$ ages of $457 \pm 5 \mathrm{ka}$ from a lava flow below and of $442 \pm 2 \mathrm{ka}$ from a relatively thin succession of fallout deposits on top (Marra et al., 2009).

\subsection{OH-DP-1955-SC5}

The tephra layer OH-DP-1955 (195.536-195.566 mcd) is $3 \mathrm{~cm}$ thick and has a sharp bottom and a diffuse top boundary. The greyish layer comprises blocky, non-vesicular glass shards and porphyritic micro-pumices containing leucite crystals. The composition is mainly phonolitic to trachyandesitic with some shards plotting in the tephriphonolitic field (Fig. 3h, Table 1). According to the stratigraphic position, OH-DP-1955 was deposited prior to ca. $460 \mathrm{ka}$, since it is found below OH-DP-1817-Pozzolane Rosse ( $457 \pm 2 \mathrm{ka})$. Proximal deposits of the active volcanoes in Italy older than 450 ka have only been explored to a limited extent. From the more distal archives, the tephra layer SC5 from the Mercure Basin (Giaccio et al., 2014) shows a trachytic-andesitic to phonolitic trend similar to that of OH-DP-1955 (cf. Fig. 3h). Oxide plots (cf. Figs. 6a and S1h) of major elements show that this composition shows a linear magmatic trend and the occurrence of two different populations of tephra can be ruled out. Investigated sources for OH-DP-1955 and its potential equivalent SC5 are very limited. Sr-isotope ratios of tephra layer SC5 reveal an origin in the Roccamonfina volcano (Giaccio et al., 2014). ${ }^{40} \mathrm{Ar} /{ }^{39} \mathrm{Ar}$ dating provided an age of $493.1 \pm 10.9 \mathrm{ka}$ for the SC5 tephra (Giaccio et al., 2014), which thus represents a poorly known eruption from the Roccamonfina volcano attributable to the Rio Rava stage dated between $\sim 600$ and $439 \mathrm{ka}$ (Rouchon et al., 2008). Despite a similar age, a potential correlation of OH-DP-1955 with Fall B ( $490 \pm 4$ ka) from the Tufi Terrosi con Pomici Bianche eruptive cycle (TTPB; $499 \pm 3-490 \pm 4 \mathrm{ka}$ ) of the Sabatini volcanic field (Sottili et al., 2004; Marra et al., 2014) is unlikely. The composition of the Fall B deposits is somehow different, having e.g. lower $\mathrm{FeO}_{\mathrm{TOT}}(\sim 1.1 \mathrm{wt} . \%)$ and $\mathrm{CaO}$ (1.5 wt.\%.) but higher $\mathrm{K}_{2} \mathrm{O}$ (3.4 wt.\%) contents (cf. Fig. 6a, Table 1).

\subsection{OH-DP-2010-Fall A}

OH-DP-2010 (201.034-201.049 mcd) is a reddish-brown, $1.5 \mathrm{~cm}$ thick layer with a sharp lower and an undulating upper boundary. It comprises morphologically different volcanic fragments, which range from non-vesicular, microlitebearing blocky glass shards to medium vesicular cuspate glass shards and elongated vesicular micro-pumices. The tephriphonolitic to phonolitic composition of tephra OH-DP2010 (Fig. 3i, Table 1) and its characteristic high potassium content suggest an origin in the Roman province. The low $\mathrm{Cl}$ content of OH-DP-2010 (cf. Table 1) is typical for Middle Pleistocene Sabatini products (Giaccio et al., 2014; Palladino et al., 2014) and supports an origin in the TTPB eruptive cycle of the Sabatini volcanic district (Sottili et al., 2004; Marra et al., 2014). The major element composition and the stratigraphic position suggest that OH-DP-2010 correlates with the proximal and distal products of the oldest eruptive unit Fall A (cf. Figs. 3i and 6b). The reddish colour of OH-DP2010 can be attributed to the reddish, terracotta-like, thermally metamorphosed clay lithics, which are a diagnostic characteristic of the proximal Fall A deposits (Sottili et al., 2004). However, the proximal deposits of Fall A apparently show a smaller compositional range (cf. Marra et al., 2014) than OH-DP-2010 and other distal archives, such as the Mercure Basin (SC3) and the Sulmona Basin (SUL 5-1c), whose correlation is corroborated by Sr-isotope analyses (Giaccio et al., 2014). Similar major element compositions (cf. Table 1, Fig. 6b) also suppose a correlation of OH-DP-2010-Fall A 


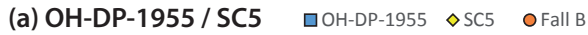

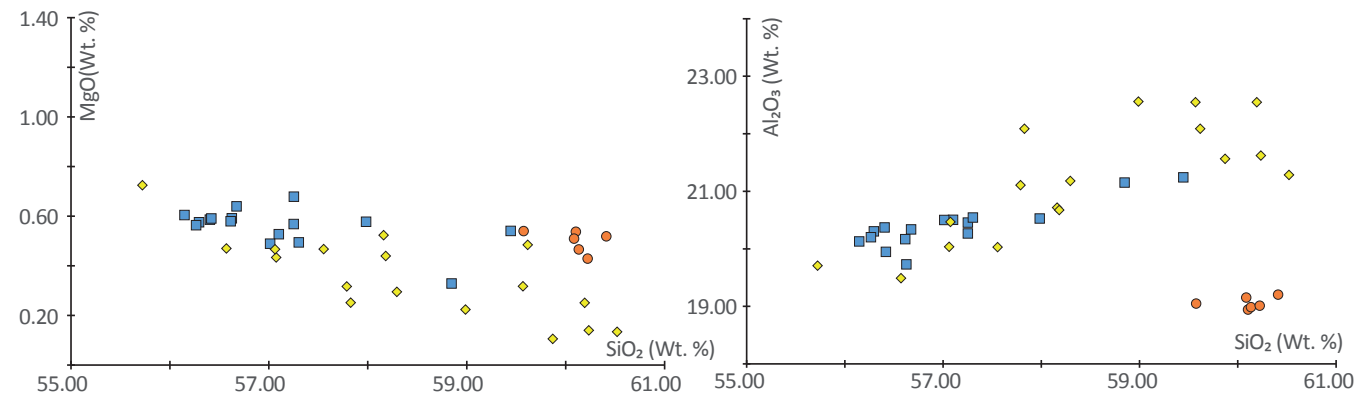

(b) OH-DP-2010/FALL A $\quad$ 口OH-DP-2010 XFALLA1 OSC3 $\triangle$ A9 OSulmona 5-1c $\quad$ FFIC-12.9
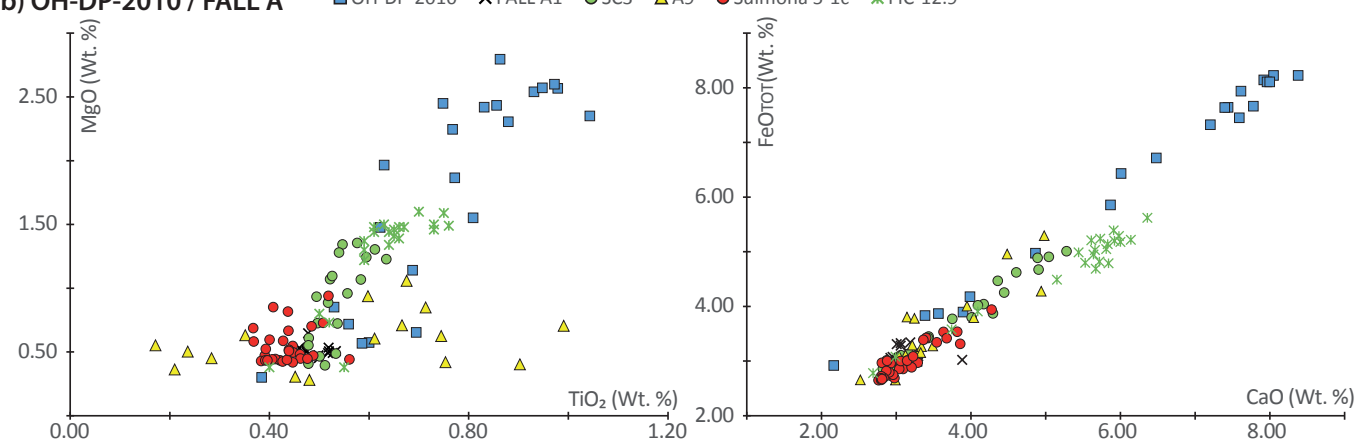

(c) OH-DP-2017 / A11/A12 $\square \mathrm{OH}-\mathrm{DP}-2017 \diamond \mathrm{A} 11 \diamond \mathrm{A} 12 * \mathrm{SC}-2$
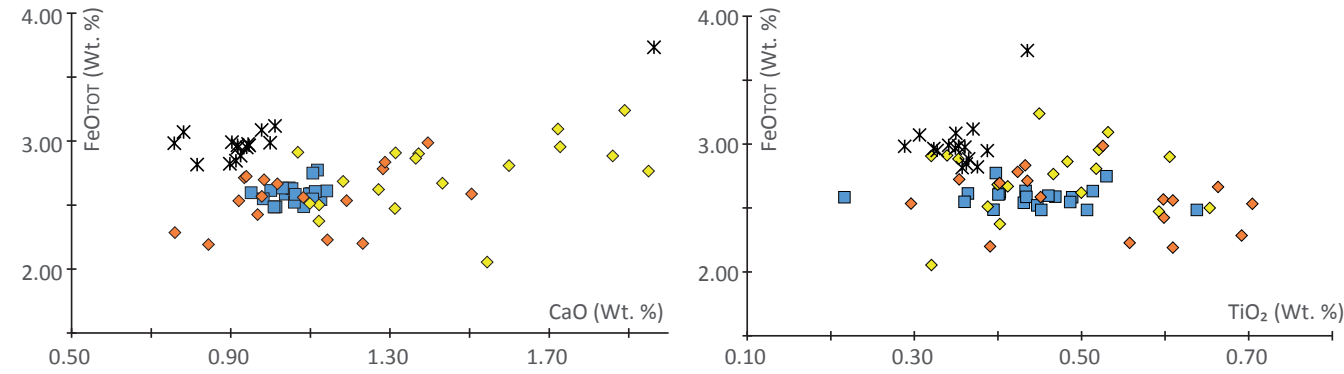

(d) OH-DP-2060 / Tufo di Bagno Albule
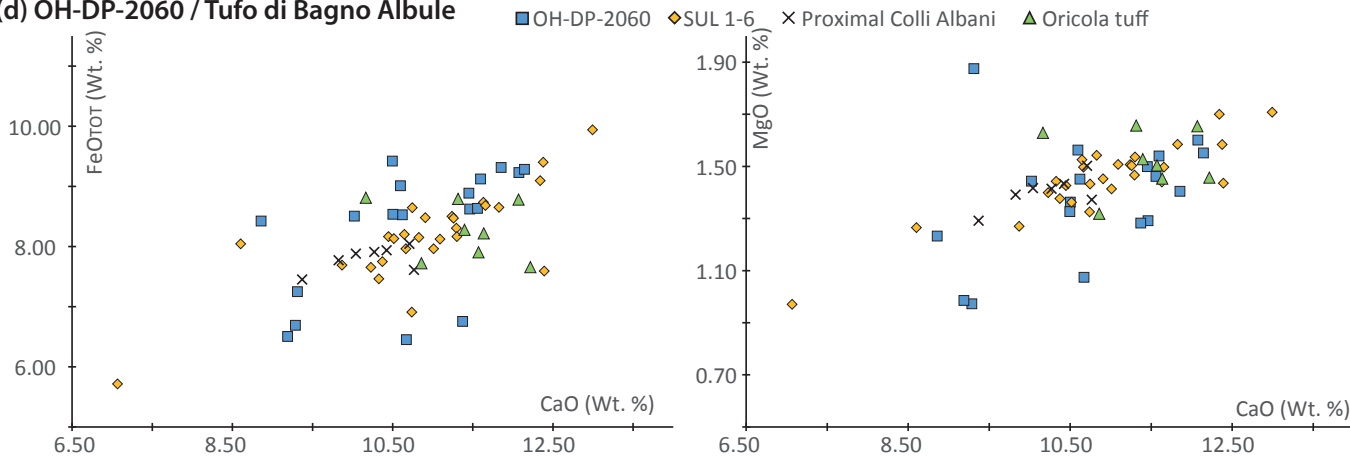

Figure 6. Harker diagrams of tephra layers OH-DP-1955 to OH-DP-2060 and their discussed equivalents. For references of correlated tephra layers, see text; additional Harker diagrams for OH-DP-1955 can be found in Fig. S1h.

with the Acerno tephra A9 (Petrosino et al., 2014b) and FIC12.9 from Ficoncella site (Aureli et al., 2015).

Sottili et al. (2004) describe a bifurcation of Fall A at the type section Isola Farnese in the Sabatini volcanic district with a lower sublayer A1 and an upper sublayer A2, both comprising alternating layers of white pumice and grey sco- ria clasts. Whereas the white pumices of these eruptive units are more evolved and trachyphonolitic, the grey scoria clasts have a phonotephritic to tephriphonolitic composition (Marra et al., 2014). The reason for the wider compositional spectrum of distal Fall A deposits (e.g. OH-DP-2010, SC3) could be the lack of data for the less evolved, upper grey pumices 
of the proximal zoned Fall A. A correlation of the older Grottarossa Pyroclastic Sequence (GRPS; Karner et al., 2001) of the Sabatini volcano (Marra et al., 2014) with the chemically similar and less evolved part of OH-DP-2010 can be excluded, since the GRPS and Fall A are temporally clearly distinct events, as shown by field (Sottili et al., 2004) and ${ }^{40} \mathrm{Ar} /{ }^{39} \mathrm{Ar}$ chronological evidence (Marra et al., 2014).

According to ${ }^{40} \mathrm{Ar} /{ }^{39} \mathrm{Ar}$ dating, the lower Fall A1 has an age of $499 \pm 3 \mathrm{ka}$ and the upper Fall A2 has an age of $496 \pm 3 \mathrm{ka}$ (Marra et al., 2014). However, unpublished agedepth interpolations from distal equivalents reveal slightly older ages, with $510 \pm 3 \mathrm{ka}$ for SC 3 at the Mercure Basin, and with $502 \pm 4 \mathrm{ka}$ for A9 at the Acerno Basin.

\subsection{OH-DP-2017-A11/A12}

Tephra layer OH-DP-2017 (201.747-201.782 mcd) is whitish to yellowish, is $3.5 \mathrm{~cm}$ thick, and has sharp bottom and top boundaries. The horizon contains highly vesicular cuspate glass shards with a varying morphology and septa thickness. The homogenous trachytic composition of $\mathrm{OH}$ DP-2017 (Fig. 3j, Table 1) excludes an origin in Roman and Roccamonfina provinces, which mainly produced phonolites and tephrophonolites during the Middle Pleistocene (cf. Giaccio et al., 2014). However, two very similar trachytic layers (A11/A12) are found in the Acerno Basin sequence (Petrosino et al., 2014b). They have a similar stratigraphic position and a similar glass chemical composition (cf. Figs. 3j and 6c, Table 1) compared with OH-DP-2017 (Petrosino et al., 2014b). An unambiguous correlation of OH-DP-2017 with one of the both layers is not possible due to their indistinguishable major element composition (Table 1). Tephra SC2 from the Sulmona Basin was tentatively also correlated based on chemical affinities and its stratigraphic position with A11/A12 (Giaccio et al., 2014). However, differences in the composition (higher $\mathrm{FeO}_{\mathrm{TOT}}$, $\mathrm{SiO}_{2}$ ) do not support an unambiguous correlation of SC2 with OH-DP-2017 or A11/12 (cf. Fig. 6c) and suggest that they may derive from the same source but from different eruptions.

According to the geochemical characteristics, an origin of A11/12 from the Campanian area was proposed (Petrosino et al., 2014b). Proximal products of the Campanian area date back to ca. 290 ka only (Seiano Ignimbrite; Rolandi et al., 2003). Distal deposits, such as found those in the Montalbano Jonico succession (around $720 \mathrm{ka}$; V5, V7; Petrosino et al., 2014c) and the Sulmona Basin (ca. $723 \mathrm{ka}$; SC135.30/SUL2-1; Giaccio et al., 2013b), also suggest earlier activity of the Campanian area, which may have produced OH-DP-2017-A11/A12. Although Sr-isotope ratios indicate that tephra layer SC2 originates from Ponza Island, the lack of Middle Pleistocene pyroclastic rocks on Ponza Island contradicts this correlation and an origin of SC2 from the Campanian area appears more likely (Giaccio et al., 2014).
Sanidine crystals of the Acerno tephra A11 yielded an ${ }^{40} \mathrm{Ar} /{ }^{39} \mathrm{Ar}$ age of $514 \pm 5.6 \mathrm{ka}$ (Petrosino et al., 2014b). As A12 is located only $12 \mathrm{~cm}$ below A11, which amounts to an age difference of a few hundred years according to the sedimentation rate between the two tephra (Petrosino et al., 2014b), the difference in age is negligible and the age of $514 \pm 5.6 \mathrm{ka}$ can be transferred from the A11 to OH-DP2017 tephra.

\subsection{OH-DP-2060-Tufo di Bagni Albule}

Tephra OH-DP-2060 (206.060-206.080 mcd) is a $2 \mathrm{~cm}$ thick, brownish-greyish tephra layer with a sharp bottom and a more diffuse upper boundary. The layer comprises mainly non-vesicular, porphyritic (leucite and sanidine), blocky glass shards. Furthermore, numerous diatoms and weathered calcite were found during microscope analysis. The characteristic K-foiditic composition (Fig. 3j, Table 1) suggests an origin in the Colli Albani volcanic district. According to the stratigraphic position of OH-DP-2060 with a presumed age older than $510 \mathrm{ka}$, it can be assigned to the Tufo del Palatino-Tufo di Bagni Albule eruptive cycle of the Colli Albani volcanic district (Karner et al., 2001; Giaccio et al., 2013a). Glass composition data are only published for the upper pyroclastic flow Tufo di Bagni Albule (TBA or Tufo di Acque Albule), which is separated by a palaeosol from the lower Tufo del Palatino (TP) ignimbrite (Marra et al., 2009). A geochemical comparison of the TBA with the proximal data of the Via Tiburtina section AH-23 (within the Colli Albani volcanic district; Marra et al., 2009) and the distal equivalent found in the Sulmona Basin (SUL1-6 site 2; Giaccio et al., 2013a) reveals a good correlation with OH-DP2060 (cf. Table 1, Fig. 6d). A relatively thick, foiditic tephra with Tufo del Palatino-Tufo di Bagni Albule products was also found in a core from the Fucino Basin in the Apennine chain (Giaccio et al., 2013a). In the intra-Apennine Carsoli Basin, a chemically very similar deposit, the so-called the Oricola tuff was found (Stoppa et al., 2005) and later correlated with TBA from the Colli Albani volcanic district based on chronological, isotopic, and major element composition affinities (Giaccio et al., 2013a). The carbonate content of the Oricola tuff (Peccerillo, 2005; Stoppa et al., 2005), which is also found in OH-DP-2060, is typical for Colli Albani products because the magma chambers are situated in Mesozoic limestones (Giordano et al., 2006). TP and TBA in proximal deposits indicate geochronologically indistinguishable ages of $530 \pm 2$ and $527 \pm 2 \mathrm{ka}$ (Marra et al., 2009), which are in a good agreement with the age obtained for the Oricola tuff (ca. $531 \mathrm{ka}$, Bosi et al., 1991). As long as no compositional glass data are available for the TP, the TBA is the most likely equivalent of OH-DP-2060 and an age of $527 \pm 2 \mathrm{ka}$ can be assumed for this tephra. 


\subsection{Reassessing and homogenizing the age of the DEEP site tephra layers}

The tephrochronological information obtained from the dated equivalents of the DEEP site tephra layers were used to develop a robust chronology for the DEEP site proxy series, for both the sediment core and the borehole successions (cf. Baumgarten et al., 2015; Francke et al., 2016). For this purpose, 11 of the 13 tephra layers presented were selected based on the strength of correlation and their geochronological reliability. Except for the Y-3, TM24a-POP2, and P-11 tephra, all ages are radiometric ages, whereas the ages of these three tephra layers were taken from the respective agedepth models. The high number of ${ }^{14} \mathrm{C}$ ages above and below the Y-3 equivalent of the Tenaghi Philippon record (Albert et al., 2015) allows a reliable age interpolation, similar to the POP2 tephra from the Sulmona Basin, where tephra layers above and below are dated precisely based on ${ }^{40} \mathrm{Ar} /{ }^{39} \mathrm{Ar}(\mathrm{Re}-$ gattieri et al., 2015). The only available ${ }^{40} \mathrm{Ar} /{ }^{39} \mathrm{Ar}$ ages of the $\mathrm{P}-11$ tephra scatter between $123 \pm 1.6 \mathrm{ka}$ and $135 \pm 1.2 \mathrm{ka}$. Currently, the most reliable age of P-11 is likely that obtained from the age-depth model of core LC21, which is tuned to the extensively U-Th-dated Soreq Cave speleothem record (Satow et al., 2015; Zanchetta et al., 2015).

In order to achieve a homogenous set of ages, all ${ }^{40} \mathrm{Ar} /{ }^{39} \mathrm{Ar}$ ages have been recalculated relative to the same flux standard and the total ${ }^{40} \mathrm{~K}$ decay constant of Steiger and Jäger (1977). Since the flux standards Fish Canyon sanidine (FCs) and Alder Creek sanidine (ACs-2) are intercalibrated, all ages were recalculated to an age of $1.194 \mathrm{Ma}$ for ACs, which corresponds to FCs at 28.02 Ma (Nomade et al., 2005). This choice was made because most of the published ages were calculated using these two flux standards. However, several calibrations of the ${ }^{40} \mathrm{Ar} /{ }^{39} \mathrm{Ar}$ chronometer are currently in use, which yield ages that can vary by $\sim 1 \%$ in the time range of the Pleistocene (e.g. Kuiper et al., 2008; Renne et al., 2010; Phillips and Matchan, 2013). As long as it is not the purpose of this work to decipher which calibration is the more accurate and as long as the implied difference in calibrated age is within the current reported fully propagated uncertainties at $2 \sigma$ level, we decided to keep ages as they were published without necessarily endorsing the flux standard values used. The results are indicated in Table 2.

The age obtained from the suggested correlation OH-DP0624-CF-V5 with the Pitigliano Tuff was recalculated, but not included in the age model, as the tephrostratigraphical correlation is not very robust (cf. discussion OH-DP-0624) and the $2 \sigma$ error bar is relatively large (Table 2). Furthermore, the age of the directly overlying tephra OH-DP-0617Vico B represents a more reliable tie point in this part of the sequence. The original (recalculated) age of Fall A, 499 (496) $\pm 3 \mathrm{ka}$, was not selected as a first-order tie point for the age-depth model shown in Francke et al. (2016) because of the age-depth interpolations of the Acerno Basin and the Mercure Basin: they suggest an older age for distal Fall A, making the published age for the proximal Fall A questionable.

In order to obtain a first overview of the chronology of the DEEP site sequence, an age-depth plot (Fig. 7) with all tephrochronological information was created. The homogenous distribution of ages vs. depth suggests a relatively constant sedimentation rate of the DEEP site succession for the upper $247.8 \mathrm{~m}$. As shown in Fig. 7, the unreliability of the ${ }^{40} \mathrm{Ar} /{ }^{39} \mathrm{Ar}$ age of Fall A at $496 \pm 3 \mathrm{ka}$ becomes more obvious. The tephra OH-DP-1955-SC5 dated to $493 \pm 11 \mathrm{ka}$ is located $5.48 \mathrm{~m}$ above OH-DP-2010-Fall A, whilst the OHDP-2017-A11 dated to $514 \pm 6 \mathrm{ka}$ is located only $0.73 \mathrm{~m}$ below this layer. Accepting an age of $496 \pm 3 \mathrm{ka}$ for Fall A would imply a distinct change in the sedimentation rates, with interpolated sedimentation rates of $1.8 \mathrm{~m} \mathrm{ka}^{-1}$ above and $0.04 \mathrm{~m} \mathrm{ka}^{-1}$ below, which differ substantially from a mean sedimentation rate of $0.39 \mathrm{~m} \mathrm{ka}^{-1}$ for the entire sequence. However, there is no lithological evidence justifying such a conspicuous change in sedimentation rate. The radiometric dating of Fall $\mathrm{A}$ was done on deposits from Cava Rinaldi, which were correlated with Fall A using only trace element ratios ( $\mathrm{Zr} / \mathrm{Y}$ vs. $\mathrm{Nb} / \mathrm{Y}$ plot) and chronological constraints because the deposits have been altered too much to use major element compositions (Marra et al., 2014). Although this method is helpful to distinguish between the different Italian provinces and between the different volcanic districts (Marra et al., 2011, 2014), it may be not suitable for distinguishing between single eruptions with similar composition. Moreover, the intense alteration of the deposits makes an unequivocal correlation difficult and the age obtained for Fall A is probably too young. Following the correlation of OH-DP-2010 with distal deposits of the SC3 in Mercure and A9 in the Acerno Basin, an age of between 502 and $510 \mathrm{ka}$ seems to be more likely and would be consistent with more constant sedimentation rates in the DEEP site record.

\subsection{Resulting tephrostratigraphy of the Lake Ohrid record}

The Middle Pleistocene Italian tephrostratigraphic framework was extended for the first time beyond Italy to the Balkan region. The 13 identified tephra layers (OH-DP0027-2060) of the DEEP sequence link the Lake Ohrid record with numerous terrestrial and marine records of the Mediterranean region (Fig. 8) at least back to MIS 13. Since the last $160 \mathrm{kyr}$ are recorded in many archives, tephra layers found in Lake Ohrid of this period were also found in numerous other records and give a dense framework of tie points for this period. However, for the period between MIS 7 and MIS 11, no correlations could be established for the Lake Ohrid sequence so far, but at least 13 tephra layers between OH-DP-0624 (earlier than $160 \mathrm{ka}$ ) and OH-DP-1817 (later than $450 \mathrm{ka}$ ) indicate activity and widespread eruptions of Italian volcanoes. On the other hand, Lake Ohrid provides a very long and continuous record of stratigraphi- 
Table 2. Depths and ages of tephra layers in the DEEP site succession. ${ }^{40} \mathrm{Ar} /{ }^{39} \mathrm{Ar}$ ages with $2 \sigma$ uncertainties (last column) recalculated to ACs at 1.194 Ma, which corresponds to FCs at 28.02 Ma (Nomade et al., 2005) and the total decay constant of Steiger and Jäger (1977). Tephra layers in bold are not considered for the age-depth modelling (Francke et al., 2016). n/a: not applicable.

\begin{tabular}{lccccc}
\hline $\begin{array}{l}\text { Tephra } \\
\text { DEEP site }\end{array}$ & $\begin{array}{l}\text { Depth } \\
(\mathrm{mcd})\end{array}$ & $\begin{array}{c}\text { Eruption/ } \\
\text { tephra }\end{array}$ & Age (ka) & References & $\begin{array}{c}\text { Recalculated } \\
40 \text { Ar/ }{ }^{39} \text { Ar ages }\end{array}$ \\
\hline OH-DP-0027 & 2.773 & Mercato & $8.43-8.63$ & Zanchetta et al. (2011) & n/a \\
OH-DP-0115 & 11.507 & Y-3 & $28.68-29.42$ & Albert et al. (2015) & n/a \\
OH-DP-0169 & 16.933 & Campanian Ignimbrite or Y-5 & $39.28 \pm 0.1$ & De Vivo et al. (2001) & $39.6 \pm 0.1$ \\
OH-DP-0404 & 40.486 & POP2 & $102 \pm 2.4$ & Regattieri et al. (2015) & n/a \\
OH-DP-0435 & 43.513 & X-6 & $109 \pm 2$ & Iori et al. (2014) & $109 \pm 2$ \\
OH-DP-0499 & 49.947 & P-11 & $133.5 \pm 2$ & Satow et al. (2015) & n/a \\
OH-DP-0617 & 61.726 & Vico Ignimbrite B & $157 \pm 3$ & Laurenzi and Villa (1987) & $162 \pm 6$ \\
OH-DP-0624 & $\mathbf{6 2 . 4 1 3}$ & Pitigliano Tuff & $\mathbf{1 5 8} \pm \mathbf{2 2}$ & Turbeville (1992a) & $\mathbf{1 6 3} \pm \mathbf{2 2}$ \\
OH-DP-1817 & 181.769 & Pozzolane Rosse & $457 \pm 4$ & Karner et al. (2001) & $457 \pm 4$ \\
& & $457 \pm 2$ & Giaccio et al. (2013) & $457 \pm 2$ \\
OH-DP-1955 & 195.566 & SC5 & $493.1 \pm 10.9$ & Giaccio et al. (2014) & $493.1 \pm 10.9$ \\
OH-DP-2010 & $\mathbf{2 0 1 . 0 4 9}$ & Sabatini Fall A & $\mathbf{4 9 9} \pm \mathbf{3}$ & Marra et al. (2014) & $\mathbf{4 9 6} \pm \mathbf{3}$ \\
OH-DP-2017 & 201.782 & Acerno A11-12 & $514 \pm 6$ & Petrosino et al. (2014) & $511 \pm 6$ \\
OH-DP-2060 & 206.080 & Tufo di Bagni Albule & $527 \pm 2$ & Marra et al. (2009) & $527 \pm 2$ \\
\hline
\end{tabular}

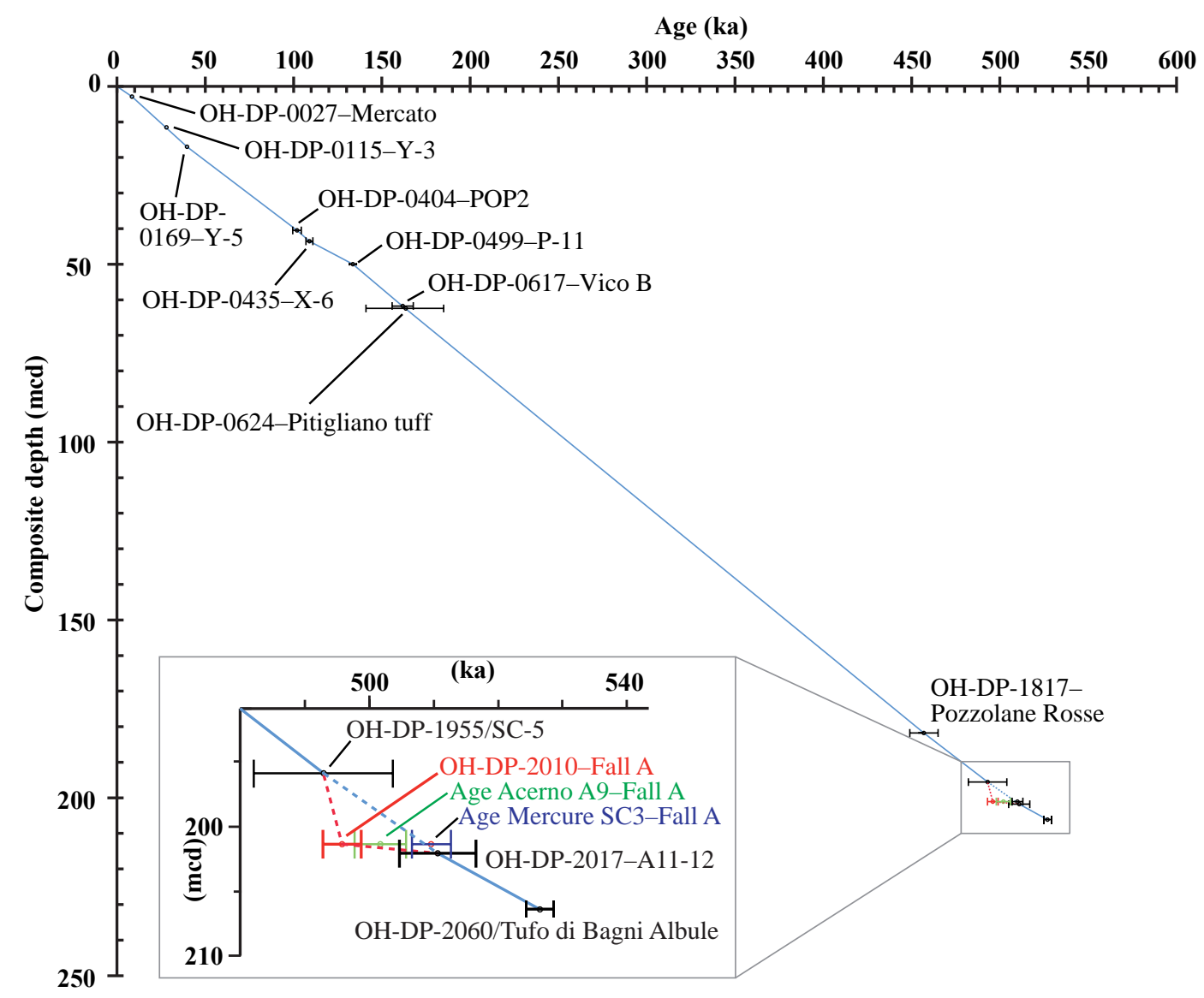

Figure 7. Age-depth plot for the selected DEEP site tephra layers. The ages of the tephra layers are based on recalculation of existing ${ }^{40} \mathrm{Ar} /{ }^{39} \mathrm{Ar}$ ages (Table 2) or are according to published data for non- ${ }^{40} \mathrm{Ar} /{ }^{39} \mathrm{Ar}$ ages. Chronological tie points were interpolated on a linear basis (blue line). The dashed lines in the insert indicate a noteworthy change in the sedimentation rate for a different age of the Fall A tephra. 


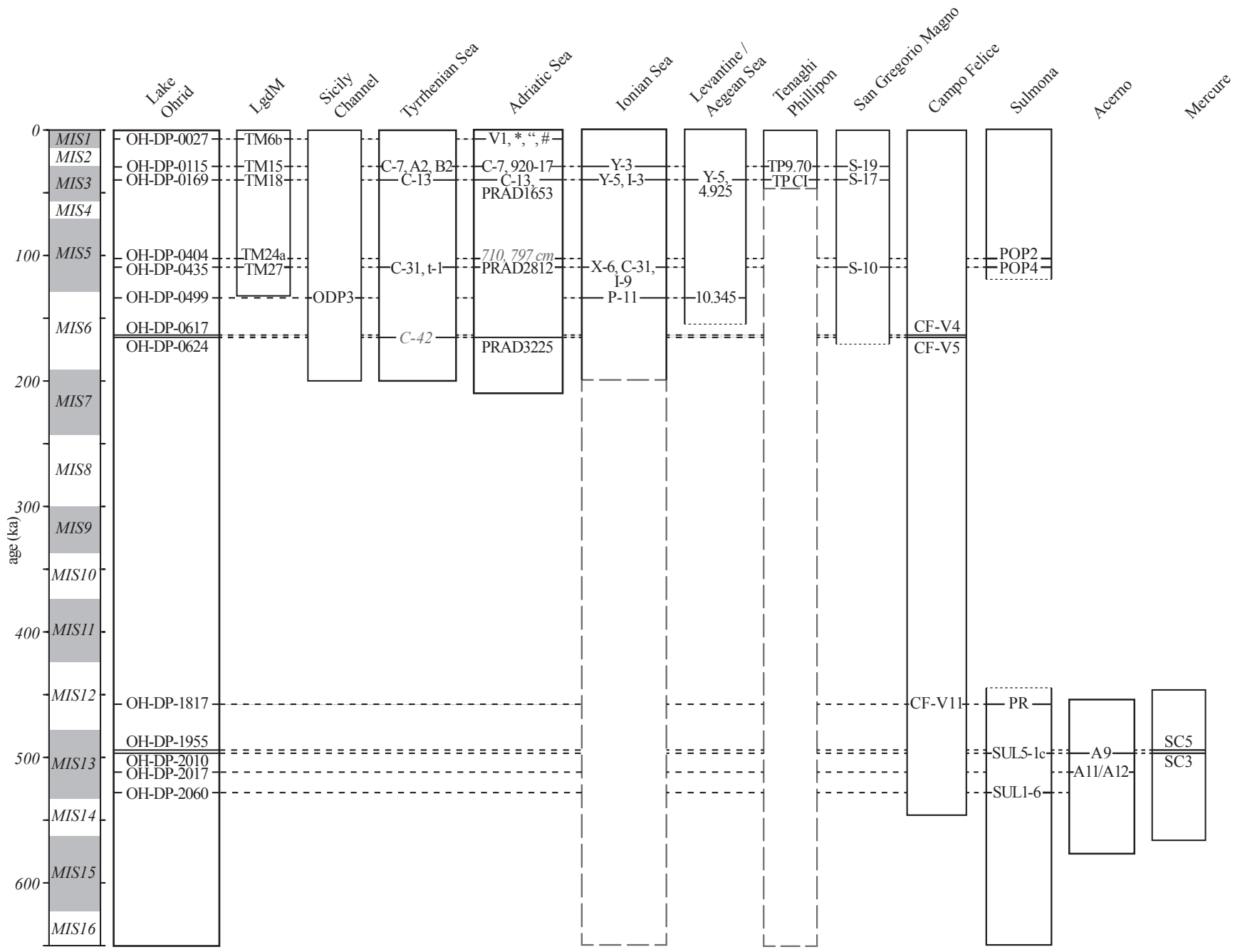

Figure 8. Tephrostratigraphic framework of Lake Ohrid and selected archives of the last 637 kyr corresponding to MIS 16 . The dashed parts of the archive columns indicate the part of the existing record where no tephrostratigraphic information is available at present. The following archives and tephra layers are presented. Lago Grande di Monticchio (LgdM; Wulf et al., 2004, 2012). Sicilian Channel: ODP963 (Tamburino et al., 2012). Tyrrhenian Sea: KET8004-DED8708, KET8003, KET8011, KET8022 (C-7, C-13, C-31, C-42; Paterne et al., 1988, 2008); C1202 (t1; Iorio et al., 2014); C45-106 (A2-B2; Munno and Petrosino, 2004). Adriatic Sea: KET8218 (V-1; Paterne et al., 1988); IN68-9, IN68-5, RF95-11, CM92-42, RF93-77 (*125-259-320 cm, 710-797 cm; Calanchi and Dinelli, 2008); AD91-17 (“190-191 to 195-196 cm; Marchini et al., 2014); MD90-917 (920-17; Zanchetta et al., 2008); MD90-918 (\#210-223 cm; Caron et al., 2012); PRAD1-2 (PRAD1653, 2812, 3225; Bourne et al., 2010, 2015). Ionian Sea: MD25/4-12 (Y-3; Albert et al., 2015); RC9-191, 22M-60, V10 69 (Y-3, Y-5, X-6; Keller et al., 1978); KET8222 (C-31, P-11; Paterne et al., 2008); KC01B (I-3, I-9; Insinga et al., 2014). Levantine Sea: RC9183, RC9-181 (Y-5; Keller et al., 1978). Aegean Sea: V10-58 (Y-5; Keller et al., 1978); LC01 (4.925, 10.345; Satow et al., 2015). Tenaghi Philippon (TP9.70, Albert et al., 2015; TP CI, Lowe et al., 2012). San Gregorio Magno Basin (Munno and Petrosino, 2007). Campo Felice Basin (Giraudi et al., 2011, 2015). Sulmona Basin (Giaccio et al., 2013b, Regattieri et al., 2015). Acerno Basin (Petrosino et al., 2013b). Mercure Basin (Giaccio et al., 2014).

cally ordered Middle Pleistocene tephra, which represents a reference section for other discontinuous and short successions in which the stratigraphic order of tephra is unknown or not well constrained. In spite of this potential, tephrostratigraphic and volcanologic implications based on the lower part of the record below P-11 are premature because the succession is not fully analysed and more detailed investigations are needed to complete and improve the tephrostratigraphic framework. Even at this preliminary stage of the study, the recognition of tephra layers from volcanic provinces which were thought to be poorly active or even inactive at the time of tephra deposition, such as the ca. $490 \mathrm{ka}$ old OH-DP1955-SC5 tephra from the Roccamonfina volcano or the ca. 510 ka old OH-DP-2017-A11/12 tephra from the Campanian Volcanic Zone, provides insights for exploring and improving our knowledge about the volcanic activity of the differ- 
ent provinces. Furthermore, their widespread distribution as far as the Balkans suggests that some of them were comparatively large-magnitude eruptions and represent widespread marker horizons.

The connection to different records within the Mediterranean region also reveals the possibility to review different distribution patterns of specific eruptions, which probably will change substantially in the light of the Lake Ohrid data (e.g. OH-DP-0499-P-11-THP-TII-5). Moreover, this established tephrostratigraphic framework can be used as a powerful tool for the synchronization of different archives in order to address relevant palaeoclimate and palaeoenvironmental issues at very fine temporal and stratigraphic resolution (cf. Zanchetta et al., 2015).

\section{Conclusions}

The results of the first tephrostratigraphic study of the DEEP site succession from Lake Ohrid allowed us to recognize 34 macroscopic tephra, of which 12 and 1 cryptotephra were correlated with their proximal or distal counterparts; all originate from Italian volcanism. Tephra layers found in the previous studies have been reidentified and were utilized as helpful markers for setting up the uppermost part of the new tephrostratigraphy of the DEEP site record. In the light of the longer tephra succession presented in this paper, the previously established correlations have been either confirmed or, in some cases, revised (e.g. OH-DP-0617-OT0701-6 and OH-DP-0624-OT07017). Furthermore, tephrostratigraphy was successfully applied for the succession older than the already inspected
135 kyr of Lake Ohrid's history. On the whole, the following correlations are proposed here: OH-DP-0027-Mercato tephra (8.43-8.63 ka cal BP), OH-DP-0115-Y-3 (28.68$29.42 \mathrm{ka}$ cal BP), OH-DP-0169-Y-5 (39.6 $\pm 0.1 \mathrm{ka})$, OHDP-0404-TM24a-POP2 (102 \pm 2.4 ka), OH-DP-0435-X-6 $(109 \pm 2 \mathrm{ka})$, OH-DP-0499-P-11 (133.5 $\pm 2 \mathrm{ka})$, OH-DP0617-Vico Ignimbrite B (162 $\pm 6 \mathrm{ka})$, OH-DP-0624-CFV5-Pitigliano Tuff (163 $\pm 22 \mathrm{ka})$, OH-DP-1817-Pozzolane Rosse $(457 \pm 2 \mathrm{ka})$, OH-DP-1955-SC5 (493.1 $\pm 10.9 \mathrm{ka})$, OH-DP-2010-Fall A (496 $\pm 3 \mathrm{ka})$, OH-DP-2017-A11/12 $(511 \pm 6 \mathrm{ka})$, and OH-DP-2060-Tufo di Bagni Albule $(527 \pm 2 \mathrm{ka})$.

The ages of 11 of the correlated tephra layers were used to contribute first-order tie points to develop a robust agedepth model of the uppermost $247.8 \mathrm{mcd}$ of the DEEP succession, which is fundamental to the development of the different multi-proxy palaeoclimatic-environmental and evolutionary studies which are in progress. Furthermore, this agedepth model has the potential to refine our chronological knowledge of some relevant marker tephra found in the Lake Ohrid succession (e.g. Fall A) and to provide a first chronological framework for a number of currently poorly known tephra, which, however, can be potentially found elsewhere and thus indirectly dated by simple geochemical fingerprinting. In this context, the data presented in this paper provide a further important step forward for extending a robust and reliable Middle Pleistocene tephrostratigraphy in the central Mediterranean area back in time and well beyond the current chronological limit. 


\section{Appendix A}

Table A1. List of the data set references used for the established correlations of the Ohrid tephra layers OH-DP-0027-OH-DP-2060.

\begin{tabular}{|c|c|c|c|}
\hline Tephra & Reference & Tephra & Reference \\
\hline OH-DP-0027 & & OH-DP-0115 & \\
\hline$\overline{\text { OT0702-3 }}$ & Vogel et al. (2010) & OT0702-4 & Sulpizio et al. (2010) \\
\hline Co1262-709 & Wagner et al. (2012) & OT0520-2 & Sulpizio et al. (2010) \\
\hline PT0915-2 & Damaschke et al. (2013) & JO187 & Sulpizio et al. (2010) \\
\hline TM-6a and TM-6b & Wulf et al. (2004) & ОT0700-1 & Sulpizio et al. (2010) \\
\hline Veliko Jezero MJ1-VJ1 & Jahns and van den Bogaard (1998) & РT0915-5 & Damaschke et al. (2013) \\
\hline KET8218 V1 & Paterne et al. (1988) & M25/4-12 Y-3 & Albert et al. (2015) \\
\hline IN68-9 $125 \mathrm{~cm}$ & Calanchi and Dinelli (2008) & RC9 191 Y-3 & Keller et al. (1978) \\
\hline IN68-5 $259 \mathrm{~cm}$ & Calanchi and Dinelli (2008) & TM-15 & Wulf et al. (2004) \\
\hline RF95-11 $320 \mathrm{~cm}$ & Calanchi and Dinelli (2008) & TP-9.70 & Albert et al. (2015) \\
\hline MD90-918 210+223 cm & Caron et al. (2012) & S19 & Munno and Petrosino (2007) \\
\hline \multirow[t]{2}{*}{ AD94-17 190-191 + 195-196cm } & Marchini et al. (2014) & $\mathrm{C} 106-\mathrm{C} 45 \mathrm{~A} 2-\mathrm{B} 2$ & Munno and Petrosino (2004) \\
\hline & & MD90-917 920-17 & Zanchetta et al. (2008) \\
\hline OH-DP-0169a+b & & $\mathrm{C}-7$ & Paterne et al. (1988) \\
\hline OT0700-2 & Sulpizio et al. (2010) & & \\
\hline OT05020-3 & Sulpizio et al. (2010) & $\underline{\mathrm{OH}-\mathrm{DP}-0404}$ & \\
\hline OT0702-6 & Sulpizio et al. (2010) & OT0702-8 & Vogel et al. (2010) \\
\hline JO-244 & Sulpizio et al. (2010) & TM24a & Wulf et al. $(2004,2012)$ \\
\hline OT0701-1/5 & Sulpizio et al. (2010) & POP2 & Regattieri et al. (2015) \\
\hline PT0704-3 & Sulpizio et al. (2010) & RF93-77 $797 \mathrm{~cm}$ & Calanchi and Dinelli (2008) \\
\hline RC9 191 Y-5 & Keller et al. (1978) & CM92-42 $710 \mathrm{~cm}$ & Calanchi and Dinelli (2008) \\
\hline $\mathrm{C}-13$ & Paterne et al. (1988) & & \\
\hline PRAD 1653 & Bourne et al. (2010) & OH-DP-0435 & \\
\hline KC01B I-3 & Insinga et al. (2014) & OT0702-9 & Vogel et al. (2010) \\
\hline LC21 4.925 & Lowe et al. (2012); Satow et al. (2015) & JO575 & Caron et al. (2012) \\
\hline TM18 & Wulf et al. (2004) & $\mathrm{X}-6$ & Keller et al. (1978) \\
\hline ML2 & Margari et al. (2007) & TM27 & Wulf et al. (2012) \\
\hline S17 & Munno and Petrosino (2007) & POP4 & Regattieri et al. (2015) \\
\hline Tenaghi Phislippon (TP) CI & Lowe et al. (2012) & S10 & Munno and Petrosino (2007) \\
\hline \multirow[t]{2}{*}{ pre-CI deposits } & Tomlinson et al. (2012) & KC01B I-9 & Insinga et al. (2014) \\
\hline & & $\mathrm{C}-31$ & Paterne et al. (2008) \\
\hline OH-DP-0499 & & PRAD2812 & Bourne et al. (2015) \\
\hline OT0702-10 & Vogel et al. (2010) & C1202 t1 & Iorio et al. (2014) \\
\hline JO941 & Caron et al. (2012) & CIL2 & Giaccio et al. (2012) \\
\hline LC21 10.345 & Satow et al. (2015) & SM1-SM2-SA & Marciano et al. (2008) \\
\hline ODP2, $-3,-4$ & Tamburrino et al. (2012) & & \\
\hline TII5 & Karkanas et al. (2015) & $\underline{\mathrm{OH}-\mathrm{DP}-0617}$ & \\
\hline ML5 & Margari et al. (2007) & OT0701-6 & Sulpizio et al. (2010) \\
\hline \multirow[t]{2}{*}{$\mathrm{P}-11$} & Paterne et al. (2008) & CF-V4 & Giraudi et al. (2011) \\
\hline & & Vico B proximal & Giraudi et al. (2011) \\
\hline \multicolumn{4}{|l|}{ OH-DP-0624 } \\
\hline OT0701-7 & Sulpizio et al. (2010) & OH-DP-1817 & \\
\hline PRAD3225 & Bourne et al. (2015) & AH-20-PRa & Marra et al. (2009) \\
\hline CF5-V5 & Giraudi and Giaccio (2015) & Sulmona Site 2 & Giaccio et al. (2013a) \\
\hline $\mathrm{C}-42$ & Paterne et al. (2008) & Sulmona Site 2a & Giaccio et al. (2013a) \\
\hline \multirow[t]{2}{*}{ Pittigliano Tuff } & Giraudi and Giaccio (2015) & Paganica site & Giaccio et al. (2013a) \\
\hline & & Raiano site 3 & Giaccio et al. (2013a) \\
\hline OH-DP-1955 & & Basal fallout & Freda et al. (2010) \\
\hline SC5 & Giaccio et al. (2014) & CF7-V11 & Giraudi and Giaccio (2015) \\
\hline \multirow[t]{2}{*}{ Fall B } & Marra et al. (2014) & & \\
\hline & & OH-DP-2010 & \\
\hline OH-DP-2017 & & Fall A proximal & Marra et al. (2014) \\
\hline A11 and A12 & Petrosino et al. (2014b) & $\mathrm{SC} 3$ & Giaccio et al. (2014) \\
\hline \multirow[t]{2}{*}{$\mathrm{SC} 2$} & Giaccio et al. (2014) & A9 & Petrosino et al. (2014b) \\
\hline & & Sulmona 5-1c & Giaccio et al. (2014) \\
\hline OH-DP-2060 & & FIC-12.9 & Aureli et al. (2015) \\
\hline SUL 1-6 & Giaccio et al. (2013a) & & \\
\hline Oricola tuff & Stoppa et al. (2005) & & \\
\hline TBA proximal & Marra et al. (2009) & & \\
\hline
\end{tabular}




\section{The Supplement related to this article is available online at doi:10.5194/bg-13-2151-2016-supplement.}

Acknowledgements. The SCOPSCO Lake Ohrid drilling campaign was funded by ICDP, the German Ministry of Higher Education and Research, the German Research Foundation, the University of Cologne, the British Geological Survey, the INGV and CNR (both Italy), and the governments of the republics of Macedonia (FYROM) and Albania. Logistic support was provided by the Hydrobiological Institute in Ohrid. Drilling was carried out by Drilling, Observation and Sampling of the Earth's Continental Crust (DOSECC) using the Deep Lake Drilling System (DLDS). Special thanks are due to Beau Marshall and the drilling team. Ali Skinner and Martin Melles provided immense help and advice during logistic preparation and the drilling operation.

Edited by: F. Wagner-Cremer

\section{References}

Aksu, A. E., Jenner, G., Hiscott, R. N., and Isler, E. B.: Occurrence, stratigraphy and geochemistry of Late Quaternary tephra layers in the Aegean Sea and the Marmara Sea, Mar. Geol., 252, 174192, 2008

Albert, P. G., Hardiman, M., Keller, J., Tomlinson, E. L., Smith, V. C., Bourne, A. J., Wulf, S., Zanchetta, G., Sulpizio, R., Muller, U. C., Pross, J., Ottolini, L., Matthews, I. P., Blockley, S. P. E., and Menzies, M. A.: Revisiting the Y-3 tephrostratigraphic marker: a new diagnostic glass geochemistry, age estimate, and details on its climatostratigraphical context, Quaternary Sci. Rev., 118, 105-121, 2015.

Baumgarten, H., Wonik, T., Tanner, D. C., Francke, A., Wagner, B., Zanchetta, G., Sulpizio, R., Giaccio, B., and Nomade, S.: Age-depth model of the past $630 \mathrm{kyr}$ for Lake Ohrid (FYROM/Albania) based on cyclostratigraphic analysis of downhole gamma ray data, Biogeosciences, 12, 7453-7465, doi:10.5194/bg-12-7453-2015, 2015.

Bear, A. N., Cas, R. A. F., and Giordano, G.: Variations in eruptive style and depositional processes associated with explosive, phonolitic composition, caldera-forming eruptions: The $151 \mathrm{ka}$ Sutri eruption, Vico Caldera, central Italy, J. Volcanol. Geoth. Res., 184, 225-255, 2009.

Bosi, C., Locardi, E., and Villa, I.: Il distretto magmatico abruzzese, Riassunti workshop "Evoluzione dei bacini Neogenici e loro rapporti con il magmatismo Plio-Quaternario nell'area ToscoLaziale”, Pisa, Italy, 12-13 June 1991, 68-69, 1991.

Bourne, A. J., Lowe, J. J., Trincardi, F., Asioli, A., Blockley, S. P. E., Wulf, S., Matthews, I. P., Piva, A., and Vigliotti, L.: Distal tephra record for the last ca 105,000 years from core PRAD 1-2 in the central Adriatic Sea implications for marine tephrostratigraphy, Quaternary Sci. Rev., 29, 3079-3094, 2010.

Bourne, A. J., Albert, P. G., Matthews, I. P., Trincardi, F., Wulf, S., Asioli, A., Blockley, S. P. E., Keller, J., and Lowe, J. J.: Tephrochronology of core PRAD 1-2 from the Adriatic Sea: insights into Italian explosive volcanism for the period $200-80 \mathrm{ka}$, Quaternary Sci. Rev., 116, 28-43, 2015.
Calanchi, N. and Dinelli, E.: Tephrostratigraphy of the last $170 \mathrm{ka}$ in sedimentary successions from the Adriatic Sea, J. Volcanol. Geoth. Res., 177, 81-95, 2008.

Calanchi, N., Cattaneo, A., Dinelli, E., Gasparotto, G., and Lucchini, F.: Tephra layers in Late Quaternary sediments of the central Adriatic Sea, Mar. Geol., 149, 191-209, 1998.

Caron, B., Sulpizio, R., Zanchetta, G., Siani, G., and Santacroce, R.: The Late Holocene to Pleistocene tephrostratigraphic record of Lake Ohrid (Albania), C. R. Geosci., 342, 453-466, 2010.

Caron, B., Siani, G., Sulpizio, R., Zanchetta, G., Paterne, M., Santacroce, R., Tema, E., and Zanella, E.: Late Pleistocene to Holocene tephrostratigraphic record from the Northern Ionian Sea, Mar. Geol., 311, 41-51, 2012.

Civetta, L., Cornette, Y., Crisci, G., Gillot, P. Y., Orsi, G., and Requejo, C. S.: Geology, Geochronology and Chemical Evolution of the Island of Pantelleria, Geol. Mag., 121, 541-562, 1984.

Civetta, L., Orsi, G., Pappalardo, L., Fisher, R. V., Heiken, G., and Ort, M.: Geochemical zoning, mingling, eruptive dynamics and depositional processes - The Campanian Ignimbrite, Campi Flegrei caldera, Italy, J. Volcanol. Geoth. Res., 75, 183-219, 1997.

Damaschke, M., Sulpizio, R., Zanchetta, G., Wagner, B., Böhm, A., Nowaczyk, N., Rethemeyer, J., and Hilgers, A.: Tephrostratigraphic studies on a sediment core from Lake Prespa in the Balkans, Clim. Past, 9, 267-287, doi:10.5194/cp-9-267-2013, 2013.

Deino, A. L., Southon, J., Terrasi, F., Campajola, L., and Orsi, G.: ${ }^{14} \mathrm{C}$ and ${ }^{40} \mathrm{Ar} /{ }^{39} \mathrm{Ar}$ Dating of the Campanian Ignimbrite, Phlegran Fields, Italy, 8th International Conference on Geochronology, Cosmochronology and Isotope Geology, Berkeley, CA, USA, Abstracts US Geol. Surv. Circ., 1107, p. 77, 1994.

De Vivo, B., Rolandi, G., Gans, P. B., Calvert, A., Bohrson, W. A., Spera, F. J., and Belkin, H. E.: New constraints on the pyroclastic eruptive history of the Campanian volcanic Plain (Italy), Miner. Petrol., 73, 47-65, 2001.

Di Vito, M. A., Sulpizio, R., Zanchetta, G., and D'Orazio, M.: The late Pleistocene pyroclastic deposits of the Campanian Plain: New insights into the explosive activity of Neapolitan volcanoes, J. Volcanol. Geoth. Res., 177, 19-48, 2008.

Druitt, T. H., Brenchley, P. J., Gokten, Y. E., and Francaviglia, V.: Late Quaternary Rhyolitic Eruptions from the Acigol Complex, Central Turkey, J. Geol. Soc. London, 152, 655-667, 1995.

Druitt, T. H., Edwards, L., Mellors, R., Pyle, D., Sparks, R., Lanphere, M., Davies, M., and Barreirio, B.: Santorini volcano, Geo. Soc. Mem., 19, 1-161, 1999.

Francke, A., Wagner, B., Just, J., Leicher, N., Gromig, R., Baumgarten, H., Vogel, H., Lacey, J. H., Sadori, L., Wonik, T., Leng, M. J., Zanchetta, G., Sulpizio, R., and Giaccio, B.: Sedimentological processes and environmental variability at Lake Ohrid (Macedonia, Albania) between $637 \mathrm{ka}$ and the present, Biogeosciences, 13, 1179-1196, doi:10.5194/bg-13-1179-2016, 2016.

Freda, C., Gaeta, M., Giaccio, B., Marra, F., Palladino, D. M., Scarlato, P., and Sottili, G.: $\mathrm{CO}_{2}$-driven large mafic explosive eruptions: the Pozzolane Rosse case study from the Colli Albani Volcanic District (Italy), B. Volcanol., 73, 241-256, 2011.

Giaccio, B., Isaia, R., Fedele, F. G., Di Canzio, E., Hoffecker, J., Ronchitelli, A., Sinitsyn, A. A., Anikovich, M., Lisitsyn, S. N., and Popov, V. V.: The Campanian Ignimbrite and Codola tephra layers: Two temporal/stratigraphic markers for the Early Upper 
Palaeolithic in southern Italy and eastern Europe, J. Volcanol. Geoth. Res., 177, 208-226, 2008.

Galli, P., Giaccio, B., and Messina, P.: The 2009 Central Italy earthquake seen through 0.5 Myr-long tectonic history of the L'Aquila faults system, Quaternary Sci. Rev., 29, 3768-3789, 2010.

Giaccio, B., Nomade, S., Wulf, S., Isaia, R., Sottili, G., Cavuoto, G., Galli, P., Messina, P., Sposato, A., Sulpizio, R., and Zanchetta, G.: The late MIS 5 Mediterranean tephra markers: a reappraisal from peninsular Italy terrestrial records, Quaternary Sci. Rev., 56, 31-45, 2012.

Giaccio, B., Arienzo, I., Sottili, G., Castorina, F., Gaeta, M., Nomade, S., Galli, P., and Messina, P.: Isotopic (Sr-Nd) and major element fingerprinting of distal tephras: an application to the Middle-Late Pleistocene markers from the Colli Albani volcano, central Italy, Quaternary Sci. Rev., 67, 190-206, 2013 a.

Giaccio, B., Castorina, F., Nomade, S., Scardia, G., Voltaggio, M., and Sagnotti, L.: Revised Chronology of the Sulmona Lacustrine Succession, Central Italy, J. Quaternary Sci., 28, 545-551, 2013b.

Giaccio, B., Galli, P., Peronace, E., Arienzo I., Nomade, S., Cavinato, G. P., Mancini, M., Messina, P., and Sottili, G.: A 560440 ka tephra record from the Mercure Basin, Southern Italy: volcanological and tephrostratigraphic implications, J. Quaternary Sci., 29, 232-248, 2014

Giaccio, B., Regattieri, E., Zanchetta, G., Nomade, S., Renne, P. R., Sprain, C. J., Drysdale, R. N., Tzedakis, P. C., Messina, P., Scardia, G., Sposato, A., and Bassinot, F.: Duration and dynamics of the best orbital analogue to the present interglacial, Geology, 43, 603-606, 2015

Giordano, G., De Benedetti, A. A., Diana, A., Diano, G., Gaudioso, F., Marasco, F., Miceli, M., Mollo, S., Cas, R. A. F., and Funiciello, R.: The Colli Albani mafic caldera (Roma, Italy): Stratigraphy, structure and petrology, J. Volcanol. Geoth. Res., 155, 4980, 2006.

Giraudi, C. and Giaccio, B.: Middle Pleistocene glaciations in the Apennines, Italy: new chronological data and preservation of the glacial record, Geo. Soc S. P., 433, doi:10.1144/SP433.1, 2015.

Giraudi, C., Bodrato, G., Lucchi, M. R., Cipriani, N., Villa, I. M., Giaccio, B., and Zuppi, G. M.: Middle and late Pleistocene glaciations in the Campo Felice Basin (central Apennines, Italy), Quaternary Res., 75, 219-230, 2011.

Grant, K. M., Rohling, E. J., Bar-Matthews, M., Ayalon, A., Medina-Elizalde, M., Ramsey, C. B., Satow, C., and Roberts, A. P.: Rapid coupling between ice volume and polar temperature over the past 150,000 years, Nature, 491, 744-747, 2012.

Hamann, Y., Wulf, S., Ersoy, O., Ehrmann, W., Aydar, E., and Schmiedl, G.: First evidence of a distal early Holocene ash layer in Eastern Mediterranean deep-sea sediments derived from the Anatolian volcanic province, Quaternary Res., 73, 497-506, 2010.

Hoffmann, N., Reicherter, K., Fernandez-Steeger, T., and Grutzner, C.: Evolution of ancient Lake Ohrid: a tectonic perspective, Biogeosciences, 7, 3377-3386, 2010.

Incarbona, A., Bonomo, S., Di Stefano, E., Zgozi, S., Essarbout, N., Talha, M., Tranchida, G., Bonanno, A., Patti, B., Placenti, F., Buscaino, G., Cuttitta, A., Basilone, G., Bahri, T., Massa, F., Censi, P., and Mazzola, S.: Calcareous nannofossil surface sediment assemblages from the Sicily Channel (central Mediter- ranean Sea): Palaeoceanographic implications, Mar. Micropaleontol., 67, 297-309, 2008.

Insinga, D. D., Tamburrino, S., Lirer, F., Vezzoli, L., Barra, M., De Lange, G. J., Tiepolo, M., Vallefuoco, M., Mazzola, S., and Sprovieri, M.: Tephrochronology of the astronomically-tuned KC01B deep-sea core, Ionian Sea: insights into the explosive activity of the Central Mediterranean area during the last $200 \mathrm{ka}$, Quaternary Sci. Rev., 85, 63-84, 2014.

Iorio, M., Liddicoat, J., Budillon, F., Incoronato, A., Coe, R. S., Insinga, D. D., Cassata, W. S., Lubritto, C., Angelino, A., and Tamburrino, S.: Combined palaeomagnetic secular variation and petrophysical records to time-constrain geological and hazardous events: An example from the eastern Tyrrhenian Sea over the last 120 ka, Global Planet. Change, 113, 91-109, 2014.

Jahns, S. and van den Bogaard, C.: New palynological and tephrostratigraphical investigations of two salt lagoons on the island of Mljet, south Dalmatia, Croatia, Veg. Hist. Archaeobot., 7, 219234, 1998.

Karkanas, P., White, D., Lane, C. S., Stringer, C., Davies, W., Cullen, V. L., Smith, V. C., Ntinou, M., Tsartsidou, G., and Kyparissi-Apostolika, N.: Tephra correlations and climatic events between the MIS6/5 transition and the beginning of MIS3 in Theopetra Cave, central Greece, Quaternary Sci. Rev., 118, 170-181, 2015.

Karner, D. B., Juvigne, E., Brancaccio, L., Cinque, A., Ermolli, E. R., Santangelo, N., Bernasconi, S., and Lirer, L.: A potential early middle Pleistocene tephrostratotype for the Mediterranean basin: the Vallo Di Diano, Campania, Italy, Global Planet. Change, 21, 1-15, 1999.

Karner, D. B., Marra, F., and Renne, P. R.: The history of the Monti Sabatini and Alban Hills volcanoes: groundwork for assessing volcanic-tectonic hazards for Rome, J. Volcanol. Geoth. Res., 107, 185-219, 2001.

Keller, J., Ryan, W. B. F., Ninkovich, D., and Altherr, R.: Explosive volcanic activity in the Mediterranean over the past 200,000 $\mathrm{yr}$ as recorded in deep-sea sediments, Geol. Soc. Am. Bull., 89, 591604, 1978.

Keller, J., Gertisser, R., Reusser, E., and Dietrich, V.: Pumice deposits of the Santorini Lower Pumice 2 eruption on Anafi island, Greece: Indications for a Plinian event of exceptional magnitude, J. Volcanol. Geoth. Res., 278, 120-128, 2014.

Kraml, M.: Laser $-{ }^{40} \mathrm{Ar} /{ }^{39} \mathrm{Ar}-$ Datierungen an distalen marinen Tephren des jung-quartären mediterranen Vulkanismus (Ionisches Meer, METEOR-Fahrt 25/4), PhD, Albert-LudwigsUniversität Freiburg i.Br. Germany, 216 pp., 1997.

Kuiper, K. F., Deino, A., Hilgen, F. J., Krijgsman, W., Renne, P. R., and Wijbrans, J. R.: Synchronizing rock clocks of Earth history, Science, 320, 500-504, 2008.

Laurenzi, M. A. and Villa, I.: ${ }^{40} \mathrm{Ar} /{ }^{39} \mathrm{Ar}$ chronostratigraphy of Vico ignimbrites, Period. Mineral., 56, 285-293, 1987.

Le Bas, M. J. L., Maitre, R. W. L., Streckeisen, A., and Zanettin, B.: A Chemical Classification of Volcanic Rocks Based on the Total Alkali-Silica Diagram, J. Petrol., 27, 745-750, 1986.

Lindhorst, K., Krastel, S., Reicherter, K., Stipp, M., Wagner, B., and Schwenk, T.: Sedimentary and tectonic evolution of Lake Ohrid (Macedonia/Albania), Basin Res., 27, 84-101, 2015.

Lourens, L. J.: Revised tuning of Ocean Drilling Program Site 964 and KC01B (Mediterranean) and implications for the $\delta^{18} \mathrm{O}$, tephra, calcareous nannofossil, and geomagnetic reversal 
chronologies of the past 1.1 Myr, Paleoceanography, 19, PA3010, doi:10.1029/2003PA000997, 2004.

Lowe, D. J.: Tephrochronology and its application: a review, Quat. Geochronol. 6, 107-153, 2011.

Lowe, J., Barton, N., Blockley, S., Ramsey, C. B., Cullen, V. L., Davies, W., Gamble, C., Grant, K., Hardiman, M., Housley, R., Lane, C. S., Lee, S., Lewis, M., MacLeod, A., Menzies, M., Müller, W., Pollard, M., Price, C., Roberts, A. P., Rohling, E. J., Satow, C., Smith, V. C., Stringer, C. B., Tomlinson, E. L., White, D., Albert, P., Arienzo, I., Barker, G., Borić, D., Carandente, A., Civetta, L., Ferrier, C., Guadelli, J.-L., Karkanas, P., Koumouzelis, M., Müller, U. C., Orsi, G., Pross, J., Rosi, M., Shalamanov-Korobar, L., Sirakov, N., and Tzedakis, P. C.: Volcanic ash layers illuminate the resilience of Neanderthals and early modern humans to natural hazards, P.Natl. Acad. Sci. USA, 109, 13532-13537, 2012.

Lustrino, M., Duggen, S., and Rosenberg, C. L.: The CentralWestern Mediterranean: Anomalous igneous activity in an anomalous collisional tectonic setting, Earth-Sci. Rev., 104, 140, 2011.

MacDonald, R.: Nomenclature and petrochemistry of the peralkaline oversaturated extrusive rocks, B. Volcanol., 38, 498-516, 1974.

Mahood, G. A. and Hildreth, W.: Geology of the peralkaline volcano at Pantelleria, Strait of Sicily, B. Volcanol., 48, 143-172, 1986.

Marchini, G., Zanchetta, G., Santacroce, R., Vigliotti, L., Capotondi, L., and Sulpizio, R.: Tephrostratigraphy of marine core AD91-17 (Adriatic Sea) Revised, Alpine and Mediterranean Quaternary, 27, 77-84, 2014.

Marciano, R., Munno, R., Petrosino, P., Santangelo, N., Santo, A., and Villa, I.: Late quaternary tephra layers along the Cilento coastline (southern Italy), J. Volcanol. Geoth. Res., 177, 227243, 2008

Margari, V., Pyle, D. M., Bryant, C., and Gibbard, P. L.: Mediterranean tephra stratigraphy revisited: Results from a long terrestrial sequence on Lesvos Island, Greece, J. Volcanol. Geoth. Res., 163, 34-54, 2007.

Marianelli, P. and Sbrana, A.: Risultati di misure di standard di minerali e di vetri naturali in microanalisi a dispersione di energia, Atti della Societa Toscana di Scienze Naturali Memorie Serie A, 105, 57-63, 1998.

Marianelli, P., Sbrana, A., and Proto, M.: Magma chamber of the Campi Flegrei supervolcano at the time of eruption of the Campanian Ignimbrite, Geology, 34, 937-940, 2006.

Marra, F., Karner, D. B., Freda, C., Gaeta, M., and Renne, P.: Large mafic eruptions at Alban Hills Volcanic District (Central Italy): Chronostratigraphy, petrography and eruptive behavior, J. Volcanol. Geoth. Res., 179, 217-232, 2009.

Marra, F., Deocampo, D., Jackson, M. D., and Ventura, G.: The Alban Hills and Monti Sabatini volcanic products used in ancient Roman masonry (Italy): An integrated stratigraphic, archaeological, environmental and geochemical approach, Earth-Sci. Rev., 108, 115-136, 2011.

Marra, F., Sottili, G., Gaeta, M., Giaccio, B., Jicha, B., Masotta, M., Palladino, D. M., and Deocampo, D. M.: Major explosive activity in the Monti Sabatini Volcanic District (central Italy) over the 800-390 ka interval: geochronological-geochemical overview and tephrostratigraphic implications, Quaternary Sci. Rev., 94, 74-101, 2014.

Matter, M., Anselmetti, F. S., Jordanoska, B., Wagner, B., Wessels, M., and Wüest, A.: Carbonate sedimentation and effects of eutrophication observed at the Kališta subaquatic springs in Lake Ohrid (Macedonia), Biogeosciences, 7, 3755-3767, doi:10.5194/bg-7-3755-2010, 2010.

Matzinger, A., Jordanoski, M., Veljanoska-Sarafiloska, E., Sturm, M., Muller, B., and Wuest, A.: Is Lake Prespa jeopardizing the ecosystem of ancient Lake Ohrid?, Hydrobiologia, 553, 89-109, 2006a.

Matzinger, A., Spirkovski, Z., Patceva, S., and Wuest, A.: Sensitivity of ancient Lake Ohrid to local anthropogenic impacts and global warming, J. Great Lakes Res., 32, 158-179, 2006 b.

Mele, D., Sulpizio, R., Dellino, P., and La Volpe, L.: Stratigraphy and eruptive dynamics of a pulsating Plinian eruption of SommaVesuvius: the Pomici di Mercato (8900 years B.P.), B. Volcanol., 73, 257-278, 2011.

Munno, R. and Petrosino, P.: New constraints on the occurence of Y-3 Upper Pleistocene tephra marker layer in the Tyrrhenian Sea, Il Quaternario, 17, 11-20, 2004.

Munno, R. and Petrosino, P.: The late Quaternary tephrostratigraphical record of the San Gregorio Magno Basin (Southern Italy), J. Quaternary Sci., 22, 247-266, 2007.

Narcisi, B. and Vezzoli, L.: Quaternary stratigraphy of distal tephra layers in the Mediterranean - an overview, Global Planet. Change, 21, 31-50, 1999.

Nomade, S., Renne, P., Vogel, N., Deino, A., Sharp, W., Becker, T., Jaouni, A., and Mundil, R.: Alder Creek sanidine (ACs-2): a Quaternary ${ }^{40} \mathrm{Ar} /{ }^{39} \mathrm{Ar}$ dating standard tied to the Cobb Mountain geomagnetic event, Chem. Geol., 218, 315-338, 2005.

Orsi, G., DeVita, S., and diVito, M.: The restless, resurgent Campi Flegrei nested caldera (Italy): Constraints on its evolution and configuration, J. Volcanol. Geoth. Res., 74, 179-214, 1996.

Palladino, D. M., Simei, S., Sottili, G., and Trigila, R.: Integrated approach for the reconstruction of stratigraphy and geology of Quaternary volcanic terrains: An application to the Vulsini Volcanoes (Central Italy), Geol. S. Am. S., 464, 63-84, 2010.

Palladino, D. M., Gaeta, M., Giaccio, B., and Sottili, G.: On the anatomy of magma chamber and caldera collapse: the example of trachy-phonolitic explosive eruptions of the Roman Province (central Italy), J. Volcanol. Geotherm. Res., 281, 12-26, 2014.

Pappalardo, L., Civetta, L., de Vita, S., Di Vito, M., Orsi, G., Carandente, A., and Fisher, R. V.: Timing of magma extraction during the Campanian Ignimbrite eruption (Campi Flegrei Caldera), J. Volcanol. Geoth. Res., 114, 479-497, 2002.

Paterne, M., Guichard, F., Labeyrie, J., Gillot, P. Y., and Duplessy, J. C.: Tyrrhenian Sea Tephrochronology of the Oxygen Isotope Record for the Past 60,000 Years, Mar. Geol., 72, 259-285, 1986.

Paterne, M., Guichard, F., and Labeyrie, J.: Explosive activity of the South Italian volcanoes during the past 80,000 years as determined by marine tephrochronology, J. Volcanol. Geoth. Res., 34, 153-172, 1988.

Paterne, M., Guichard, F., Duplessy, J. C., Siani, G., Sulpizio, R., and Labeyrie, J.: A 90,000-200,000 yrs marine tephra record of Italian volcanic activity in the Central Mediterranean Sea, J. Volcanol. Geoth. Res., 177, 187-196, 2008. 
Peccerillo, A.: Plio-Quaternary Volcanism in Italy: Petrology, Geochemistry, Geodynamics, Springer-Verlag Berlin Heidelberg, Berlin, Germany, 2005.

Petrosino, P., Ermolli, E. R., Donato, P., Jicha, B., Robustelli, G., and Sardella, R.: Using Tephrochronology and palynology to date the MIS 13 lacustrine sediments of the Mercure Basin (Southern Apennines-Italy), Italian Journal of Geosciences, 133, 169-186, 2014a.

Petrosino, P., Jicha, B. R., Mazzeo, F. C., and Russo Ermolli, E.: A high resolution tephrochronological record of MIS 14-12 in the Southern Apennines (Acerno Basin, Italy), J. Volcanol. Geoth. Res., 274, 34-50, 2014b.

Petrosino, P., Jicha, B. R., Mazzeo, F. C., Ciaranfi, N., Girone, A., Maiorano, P., and Marino, M.: The Montalbano Jonico marine succession: An archive for distal tephra layers at the EarlyMiddle Pleistocene boundary in southern Italy, Quatern. Int., 383, 89-103, 2015.

Phillips, D. and Matchan, E.: Ultra-high precision ${ }^{40} \mathrm{Ar} /{ }^{39} \mathrm{Ar}$ ages for Fish Canyon Tuff and Alder Creek Rhyolite sanidine: New dating standards required?, Geochim. Cosmochim. Ac., 121, 229-239, 2013.

Pross, J., Tzedakis, P., Schmiedl, G., Christanis, K., Hooghiemstra, H., Müller, U. C., Kotthoff, U., Kalaitzidis, S., and Milner, A.: Tenaghi Philippon (Greece) Revisited: Drilling a Continuous Lower-Latitude Terrestrial Climate Archive of the Last 250,000 Years, Sci. Dril., 5, 44-46, doi:10.2204/iodp.sd.5.06.2007, 2007.

Pyle, D. M., Ricketts, G. D., Margari, V., van Andela, T. H., Sinitsyn, A. A., Praslov, N. D., and Lisitsyn, S.: Wide dispersal and deposition of distal tephra during the Pleistocene "Campanian Ignimbrite/Y5” eruption, Italy, Quaternary Sci. Rev., 25, 27132728, 2006.

Regattieri, E., Giaccio, B., Zanchetta, G., Drysdale, R. N., Galli, P., Nomade, S., Peronace, E., and Wulf, S.: Hydrological variability over the Apennines during the Early Last Glacial precession minimum, as revealed by a stable isotope record from Sulmona basin, Central Italy, J. Quaternary Sci., 30, 19-31, 2015.

Regattieri, E., Giaccio, B., Galli, P., Nomade, S., Peronace, E., Messina P., Sposato, A., Boschi, C., and Gemelli, M.: A multiproxy record of MIS 11-12 deglaciation and glacial MIS 12 instability from the Sulmona Basin (central Italy), Quaternary Sci. Rev. 132, 129-145, 2016.

Reicherter, K., Hoffmann, N., Lindhorst, K., Krastel, S., FernándezSteeger, T., Grützner, C., and Wiatr, T.: Active basins and neotectonics: morphotectonics of the Lake Ohrid Basin (FYROM and Albania), Z. Dtsch. Ges. Geowiss., 162, 217-234, 2011.

Renne, P. R., Mundil, R., Balco, G., Min, K., and Ludwig, K. R.: Joint determination of $40 \mathrm{~K}$ decay constants and $40 \mathrm{Ar} * / 40 \mathrm{~K}$ for the Fish Canyon sanidine standard, and improved accuracy for ${ }^{40} \mathrm{Ar} /{ }^{39} \mathrm{Ar}$ geochronology, Geochim. Cosmochim. Ac., 74, 5349-5367, 2010.

Robertson, A. and Shallo, M.: Mesozoic-Tertiary tectonic evolution of Albania in its regional Eastern Mediterranean context, Tectonophysics, 316, 197-254, 2000.

Rolandi, G., Bellucci, F., Heizler, M. T., Belkin, H. E., and De Vivo, B.: Tectonic controls on the genesis of ignimbrites from the Campanian Volcanic Zone, southern Italy, Miner. Petrol., 79, 3-31, 2003.

Rotolo, S. G., Scaillet, S., La Felice, S., and Vita-Sceillet, G.: A revision of the structure and stratigraphgy of pre-Green Tuff ign- imbrites at Pantelleria (Strait of Silicy), J. Volcanol. Geoth. Res., 250, 61-74, 2013.

Rouchon, V., Gillot, P. Y., Quidelleur, X., Chiesa, S., and Floris, B.: Temporal evolution of the Roccamonfina volcanic complex (Pleistocene), Central Italy, J. Volcanol. Geoth. Res., 177, 500 514, 2008.

Roulleau, E., Pinti, D. L., Rouchon, V., Quidelleur, X., and Gillot, P.-Y.: Tephro-chronostratigraphy of the lacustrine interglacial record of Piànico, Italian Southern Alps: Identifying the volcanic sources using radiogenic isotopes and trace elements, Quatern. Int., 204, 31-43, 2009.

Russo Ermolli, E., Aucelli, P. P. C., Di Rollo, A., Mattei, M., Petrosino, P., Porreca, M., and Rosskopf, C. M.: An integrated stratigraphical approach to the Middle Pleistocene succession of the Sessano basin (Molise, Italy), Quatern. Int., 225, 114-127, 2010.

Sagnotti, L., Scardia, G., Giaccio, B., Liddicoat, J. C., Nomade, S., Renne, P. R., and Sprain, C. J.: Extremely rapid directional change during Matuyama-Brunhes geomagnetic polarity reversal, Geophys. J. Int., 199, 1110-1124, 2014.

Santacroce, R., Cioni, R., Marianelli, P., Sbrana, A., Sulpizio, R., Zanchetta, G., Donahue, D. J., and Joron, J. L.: Age and whole rock-glass compositions of proximal pyroclastics from the major explosive eruptions of Somma-Vesuvius: A review as a tool for distal tephrostratigraphy, J. Volcanol. Geoth. Res., 177, 1-18, 2008.

Sarna-Wojcicki, A.: Tephrochronology, in: Quaternary Geochronology, edited by: Noller, J. S., Sowers, J. M., and Lettis, W. R., AGU Reference Shelf, American Geophysical Union, Washington, DC, USA, 2013.

Satow, C., Tomlinson, E. L., Grant, K. M., Albert, P. G., Smith, V. C., Manning, C. J., Ottolini, L., Wulf, S., Rohling, E. J., Lowe, J. J., Blockley, S. P. E., and Menzies, M. A.: A new contribution to the Late Quaternary tephrostratigraphy of the Mediterranean: Aegean Sea core LC21, Quaternary Sci. Rev., 117, 96-112, 2015.

Scaillet, S., Vita-Sceillet, G., and Rotolo, S. G.: Millennial-scale phase relationshhips between ice-core and Mediterranean marine records: insights from high-precision ${ }^{40} \mathrm{Ar} /{ }^{39} \mathrm{Ar}$ dating of the Green Tuff of Pantelleria, Silicy Strait, Quaternary Sci. Rev., 78, 141-154, 2013.

Siani, G., Sulpizio, R., Paterne, M., and Sbrana, A.: Tephrostratigraphy study for the last 18,000 C-14 years in a deep-sea sediment sequence for the South Adriatic, Quaternary Sci Rev, 23, 2485 2500, 2004.

Sottili, G., Palladino, D. M., and Zanon, V.: Plinian activity during the early eruptive history of the Sabatini volcanic district, central Italy, J. Volcanol. Geoth. Res., 135, 361-379, 2004.

Steiger, R. H. and Jäger, E.: Subcommission on geochronology: Convention on the use of decay constants in geo- and cosmochronology, Earth Planet. Sc. Lett., 36, 359-362, 1977.

Stoppa, F., Rosatelli, G., Wall, F., and Jeffries, T.: Geochemistry of carbonatite-silicate pairs in nature: A case history from Central Italy, Lithos, 85, 26-47, 2005.

St. Seymour, K., Christanis, K., Bouzinos, A., Papazisimou, S., Papatheodorou, G., Moran, E., and Denes, G.: Tephrostratigraphy and tephrochronology in the Philippi peat basin, Macedonia, Northern Hellas (Greece), Quatern. Int., 121, 53-65, 2004.

Sulpizio, R., Zanchetta, G., Paterne, M., and Siani, G.: A review of tephrostratigraphy in central and southern Italy during the 
last 65 ka, Il Quaternario Italian, J. Quaternary Sci., 16, 91-108, 2003.

Sulpizio, R., Bonasia, R., Dellino, P., Di Vito, M. A., La Volpe, L., Mele, D., Zanchetta, G., and Sadori, L.: Discriminating the long distance dispersal of fine ash from sustained columns or near ground ash clouds: The example of the Pomici di Avellino eruption (Somma-Vesuvius, Italy), J. Volcanol. Geoth. Res., 177, 263-276, 2008.

Sulpizio, R., Zanchetta, G., D’Orazio, M., Vogel, H., and Wagner, B.: Tephrostratigraphy and tephrochronology of lakes Ohrid and Prespa, Balkans, Biogeosciences, 7, 3273-3288, doi:10.5194/bg7-3273-2010, 2010.

Sulpizio, R., Alcicek, M. C., Zanchetta, G., and Solari, L.: Recognition of the Minoan tephra in the Acigöl Basin, Western Turkey: implications for inter-archive correlations and fine ash dispersal, J. Quaternary Sci., 28, 329-335, 2013.

Sumita, M. and Schmincke, H.-U.: Impact of volcanism on the evolution of Lake Van II: Temporal evolution of explosive volcanism of Nemrut Volcano (eastern Anatolia) during the past ca. 0.4 Ma, J. Volcanol. Geoth. Res., 253, 15-34, 2013.

Tamburrino, S., Insinga, D. D., Sprovieri, M., Petrosino, P., and Tiepolo, M.: Major and trace element characterization of tephra layers offshore Pantelleria Island: insights into the last $200 \mathrm{ka}$ of volcanic activity and contribution to the Mediterranean tephrochronology, J. Quaternary Sci., 27, 129-140, 2012.

Thunell, R., Federman, A., Sparks, S., and Williams, D.: Age, origin and volcanological significance of the Y-5 ash layer in the Mediterranean, Quaternary Res., 12, 241-253, 1979.

Tomlinson, E. L., Arienzo, I., Civetta, L., Wulf, S., Smith, V. C., Hardiman, M., Lane, C. S., Carandente, A., Orsi, G., Rosi, M., Müller, W., and Menzies, M. A.: Geochemistry of the Phlegraean Fields (Italy) proximal sources for major Mediterranean tephras: Implications for the dispersal of Plinian and co-ignimbritic components of explosive eruptions, Geochim. Cosmochim. Ac., 93, 102-128, 2012.

Tomlinson, E. L., Smith, V. C., Albert, P. G., Aydar, E., Civetta, L., Cioni, R., Çubukçu, E., Gertisser, R., Isaia, R., Menzies, M. A., Orsi, G., Rosi, M., and Zanchetta, G.: The major and trace element glass compositions of the productive Mediterranean volcanic sources: tools for correlating distal tephra layers in and around Europe, Quaternary Sci. Rev., 118, 48-66, 2015.

Ton-That, T., Singer, B., and Paterne, M.: ${ }^{40} \mathrm{Ar} /{ }^{39} \mathrm{Ar}$ dating of latest Pleistocene ( $41 \mathrm{ka}$ ) marine tephra in the Mediterranean Sea: implications for global climate records, Earth Planet. Sc. Lett., 184, 645-658, 2001.

Turbeville, B. N.: Tephra Fountaining, Rheomorphism, and Spatter Flow during Emplacement of the Pitigliano Tuffs, LateraCaldera, Italy, J. Volcanol. Geoth. Res., 53, 309-327, 1992a.

Turbeville, B. N.: ${ }^{40} \mathrm{Ar} /{ }^{39} \mathrm{Ar}$ Ages and stratigraphy of the Latera caldera, Italy, B. Volcanol., 55, 110-118, 1992b.

Tzedakis, P. C.: Long-term tree populations in northwest Greece through multiple Quaternary climatic cycles, Nature, 364, 437440, 1993.

Vezzoli, L.: Tephra Layers in Bannock Basin (Eastern Mediterranean), Mar. Geol., 100, 21-34, 1991.

Villa, I. M. and Buettner, A.: Chronostratigraphy of Monte Vulture volcano (southern Italy): secondary mineral microtextures and ${ }^{40} \mathrm{Ar} /{ }^{39} \mathrm{Ar}$ systematics, B. Volcanol., 71, 1195-1208, 2009.
Vogel, H., Zanchetta, G., Sulpizio, R., Wagner, B., and Nowaczyk, N.: A tephrostratigraphic record for the last glacial-interglacial cycle from Lake Ohrid, Albania and Macedonia, J. Quaternary Sci., 25, 320-338, 2010.

Wagner, B., Reicherter, K., Daut, G., Wessels, M., Matzinger, A., Schwalb, A., Spirkovski, Z., and Sanxhaku, M.: The potential of Lake Ohrid for long-term palaeoenvironmental reconstructions, Palaeogeogr. Palaeocl., 259, 341-356, 2008.

Wagner, B., Vogel, H., Zanchetta, G., and Sulpizio, R.: Environmental change within the Balkan region during the past ca. $50 \mathrm{ka}$ recorded in the sediments from lakes Prespa and Ohrid, Biogeosciences, 7, 3187-3198, doi:10.5194/bg-7-3187-2010, 2010.

Wagner, B., Francke, A., Sulpizio, R., Zanchetta, G., Lindhorst, K., Krastel, S., Vogel, H., Rethemeyer, J., Daut, G., Grazhdani, A., Lushaj, B., and Trajanovski, S.: Possible earthquake trigger for 6th century mass wasting deposit at Lake Ohrid (Macedonia/Albania), Clim. Past, 8, 2069-2078, doi:10.5194/cp-8-20692012, 2012.

Wagner, B., Wilke, T., Krastel, S., Zanchetta, G., Sulpizio, R., Reicherter, K., Leng, M. J., Grazhdani, A., Trajanovski, S., Francke, A., Lindhorst, K., Levkov, Z., Cvetkoska, A., Reed, J. M., Zhang, X., Lacey, J. H., Wonik, T., Baumgarten, H., and Vogel, H.: The SCOPSCO drilling project recovers more than 1.2 million years of history from Lake Ohrid, Sci. Dril., 17, 19-29, doi:10.5194/sd-17-19-2014, 2014.

Washington, H. S.: The Roman comagmatic region, Carnegie Institution of Washington, Washington, DC, 1906.

Watzin, M. C., Puka, V., and Naumoski, T. B.: Lake Ohrid and Its Watershed: Our Lake, Our Future, A State of the Environment Report, Tirana, Albania and Ohrid, Macedonia, 134 pp., 2002.

Wulf, S., Kraml, M., Brauer, A., Keller, J., and Negendank, J. F. W.: Tephrochronology of the 100ka lacustrine sediment record of Lago Grande di Monticchio (Southern Italy), Quatern. Int., 122, 7-30, 2004.

Wulf, S., Brauer, A., Mingram, J., Zolitschka, B., and Negendank, J. F. W.: Distal tephras in the sediments of Monticchio maar lakes, Principe, C., Regione Basilicata, Dipartimento Ambiente, Territorio e Politiche della Sostenibilità, 2006.

Wulf, S., Keller, J., Paterne, M., Mingram, J., Lauterbach, S., Opitz, S., Sottili, G., Giaccio, B., Albert, P. G., Satow, C., Tomlinson, E. L., Viccaro, M., and Brauer, A.: The 100-133 ka record of Italian explosive volcanism and revised tephrochronology of Lago Grande di Monticchio, Quaternary Sci. Rev., 58, 104-123, 2012.

Zanchetta, G., Sulpizio, R., Giaccio, B., Siani, G., Paterne, M., Wulf, S., and D'Orazio, M.: The Y-3 tephra: A Last Glacial stratigraphic marker for the Central Mediterranean Basin, J. Volcanol. Geoth. Res., 177, 145-154, 2008.

Zanchetta, G., Sulpizio, R., Roberts, N., Cioni, R., Eastwood, W. J., Siani, G., Caron, B., Paterne, M., and Santacroce, R.: Tephrostratigraphy, chronology and climatic events of the Mediterranean basin during the Holocene: An overview, Holocene, 21, 33-52, 2011.

Zanchetta, G., Regattieri, E., Giaccio, B., Wagner, B., Sulpizio, R., Francke, A., Vogel, L. H., Sadori, L., Masi, A., Sinopoli, G., Lacey, J. H., Leng, M. L., and Leicher, N.: Aligning MIS5 proxy records from Lake Ohrid (FYROM) with independently dated Mediterranean archives: implications for core chronology, Biogeosciences Discuss., 12, 16979-17007, doi:10.5194/bgd12-16979-2015, 2015. 